\title{
GROUND-WATER ALTITUDES AND WELL DATA, NYE COUNTY, NEVADA, AND \\ INYO COUNTY, CALIFORNIA
}

by Marek S. Ciesnik

\section{U.S. GEOLOGICAL SURVEY}

Open-File Report 93-89

Prepared in cooperation with the

NEVADA OPERATIONS OFFICE,

U.S. DEPARTMENT OF ENERGY under

Interagency Agreement DE-Al08-92NV10874

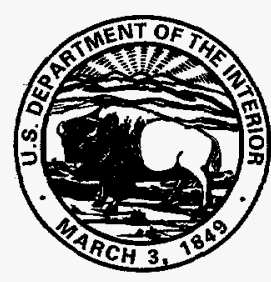




\section{U.S. DEPARTMENT OF THE INTERIOR \\ BRUCE BABBITT, Secretary}

U.S. GEOLOGICAL SURVEY

Gordon P. Eaton, Director

The use of trade, product, industry, or firm names is for descriptive purposes only and does not imply endorsement by the U.S. Government.

For additional information write to:

Chief, Earth Science Investigations

Program

Yucca Mountain Project Branch

U.S. Geological Survey

Box 25046, MS 421

Denver Federal Center

Denver, CO 80225
Copies of this report can be purchased from:

U.S. Geological Survey Earth Science Information Center

Open-File Reports Section

Box 25286, MS 517

Denver Federal Center

Denver, CO 80225 


\section{CONTENTS}

Abstract

Introduction

Ground-water data

References cited.

\section{PLATES}

[In pocket]

1. Map showing the location of data-base wells in the Beatty, Nevada-California quadrangle

2. Map showing the location of data-base wells in the Death Valley Junction, California-Nevada quadrangle

\section{FIGURE}

1. Map showing study area with generalized well locations

\section{TABLES}

1. Ground-water levels and well data of wells located in the Beatty, Nevada-California, 30-minute $\times 60$-minute quadrangle

2. Ground-water levels and well data of wells located in the Death Valley Junction, California-Nevada, 30 -minute $\times 60$-minute quadrangle.

CONVERSION FACTORS AND VERTICAL DATUM

\begin{tabular}{rll}
\hline Multiply & By & To obtain \\
\hline kilometer $(\mathrm{km})$ & & mile \\
meter $(\mathrm{m})$ & 0.6214 & foot \\
inch \\
centimeter $(\mathrm{cm})$ & 0.391 & square mile \\
square kilometer $\left(\mathrm{km}^{2}\right)$ & 0.386 & acre-foot \\
\hline cubic meter $\left(\mathrm{m}^{3}\right)$ & 0.0008107 & \\
\hline
\end{tabular}

Sea level: In this report "sea level" refers to the National Geodetic Vertical Datum of 1929 (NGVD of 1929)—a geodetic datum derived from a general adjustment of the first-order level nets of both the United States and Canada, formerly called Sea Level Datum of 1929.

\section{DISCLAIMER}

This report was prepared as an account of work sponsored by an agency of the United States Government. Neither the United States Government nor any agency thereof, nor any of their employees, makes any warranty, express or implied, or assumes any legal liability or responsibility for the accuracy, completeness, or usefulness of any information, apparatus, product, or process disclosed, or represents that its use would not infringe privately owned rights. Reference herein to any specific commercial product, process, or service by trade name, trademark, manufacturer, or otherwise does not necessarily constitute or imply its endorsement, recommendation, or favoring by the United States Government or any agency thereof. The views and opinions of authors expressed herein do not necessarily state or reflect those of the 


\section{DISCLAIMER}

Portions of this document may be illegible in electronic image products. Images are produced from the best available original document. 


\title{
Ground-Water Altitudes and Well Data, Nye County, Nevada, and Inyo County, California
}

\author{
ByMarek S. Ciesnik
}

\begin{abstract}
This report contains ground-water altitudes and well data for wells located in Nye County, Nevada, and Inyo County, California, south of Yucca Mountain, Nevada, the potential site for a high-level nuclear waste repository. Data are from wells whose coordinates are within the Beatty and Death Valley Junction, California-Nevada maps from the U.S. Geological Survey, scale 1:100,000 (30-minute $\times 60$-minute quadrangle). Compilation of these data was made to provide a reference for numerical models of ground-water flow at Yucca Mountain and its vicinity. Water-level measurements were obtained from the U.S. Geological Survey National Water Information System (NWIS) data base, and span the period of October 1951 to May 1991; most measurements were made from 1980 to 1990 .
\end{abstract}

\section{INTRODUCTION}

This report presents a comprehensive list of water levels recorded in the U.S. Geological Survey National Water Information System (NWIS) data base for the area shown in figure 1 . The area of interest encompasses about $3,500 \mathrm{~km}^{2}$ in southern Nevada and southeastern California and includes the Amargosa Desert, the southern part of the Funeral Mountains, the southern part of Oasis Valley, Crater Flat, Yucca Mountain and the southern part of the Nevada Test Site, and the northern end of Pahrump Valley. The water levels tabulated in this report were measured from October 1951 to May 1991. Most of the water levels were measured from 1980 to 1990 , and the measurements from earlier dates are added for more complete areal coverage.

Characterization of Yucca Mountain as a potential site for a nuclear waste repository has resulted in a number of ground-water studies in this region during the last few years. These studies include those by Kilroy (1991), McKinley and others (1991), Oatfield and Czarnecki (1989), Nichols and Akers (1985), and
Czarnecki and Waddell (1984). Various aspects of changes in ground-water altitudes in the Amargosa Desert are discussed by Kilroy (1991) and by Nichols and Akers (1985).

\section{GROUND-WATER DATA}

With the exception of the site number, the USGS NWIS data base is the source of the data presented in tables 1 and 2. Site numbers represent arbitrarily assigned numbers for ease of reference used in the USGS regional ground-water-modeling activity within the Yucca Mountain Project. Site-ID, formatted as a 15-digit number, uniquely identifies sites in the NWSI data base. The first 13 digits of the Site-ID numbers originate from the initial determination of the site latitude and longitude and are not changed with subsequent refinement of well locations. The last two digits are assigned sequentially to distinguish among sites that may be within 1 second of the same location. Depth of well and depth to water are given in meters below land surface. Data in tables 1 and 2 were checked by computer through location plotting, waterlevel contouring, and comparison against a file of known errors compiled through years of utilization of these data. A detailed description of the NWIS system is contained in volume 2 , chapter 4 of the USGS OpenFile Report 89-587 ("Ground-Water Site Inventory System").

Water-level and well data from 541 wells, located in the area adjacent to Yucca Mountain are presented in tables 1 and 2 . The locations of these wells are plotted on plate 1 (Beatty, Nevada-California $30^{\prime} \times 60^{\prime}$ quadrangle; USGS, 1986) and plate 2 (Death Valley Junction, California-Nevada $30^{\prime} \times 60^{\prime}$ quadrangle; USGS, 1986).

Three socioeconomic factors seem to have had an effect on the natural ground-water potentiometric surface in the study area: (1) Changes in population; (2) agricultural activities; and (3) mining activities. Although a detailed analysis of these factors is beyond the scope of this report, some relevant figures are presented below to illustrate the phenomenon. 


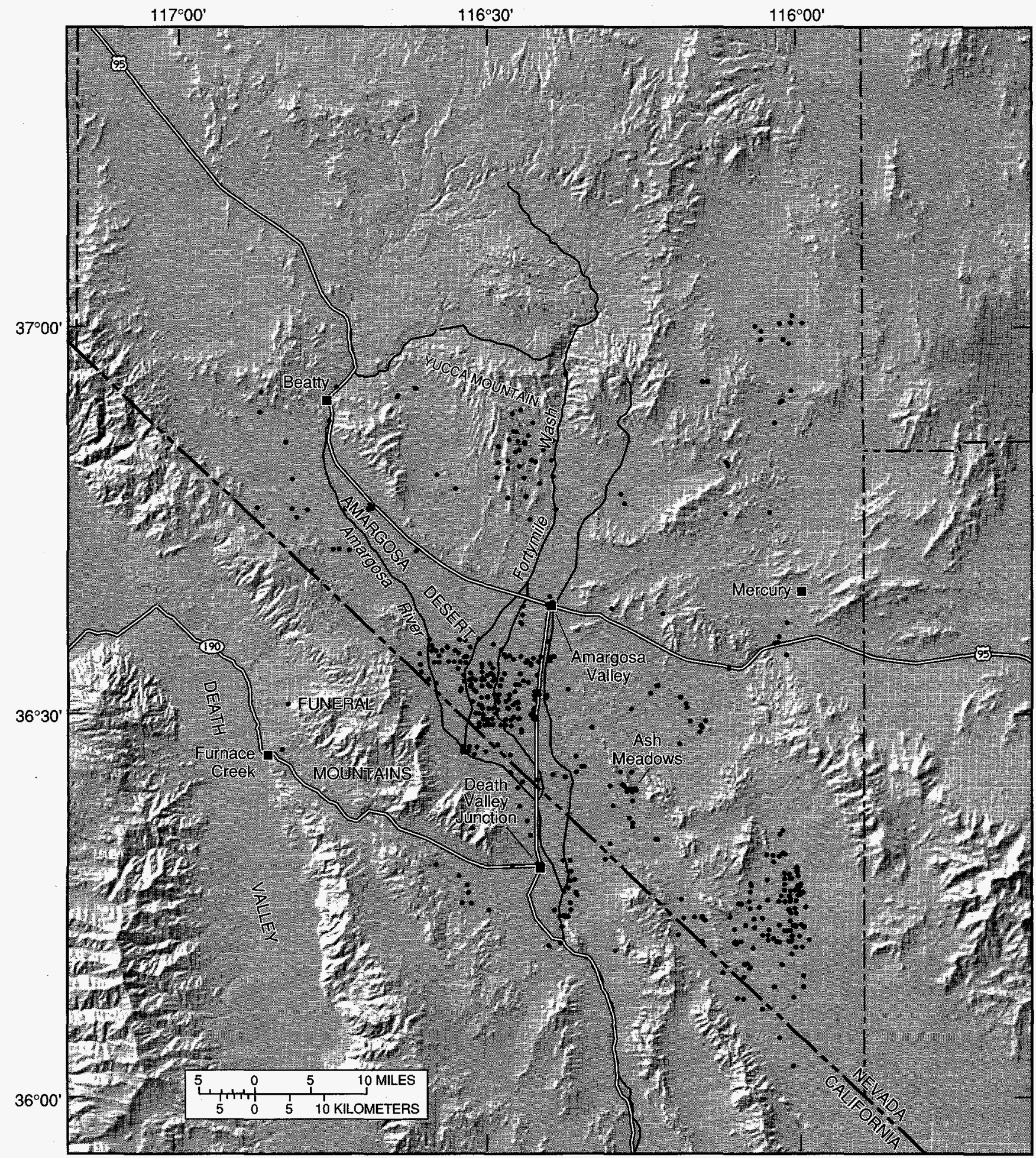

Base from U.S. Geological Survey digital elevation data, 1:250,000, 1987, and digital data, 1:100,000, 1981-89; Universal Transverse Mercator projection, Zone 11. Shaded-relief base from 1:250,000-scale Digital Elevation Model; sun illumination from northwest at 30

degrees above horizon

EXPLANATION

- Well

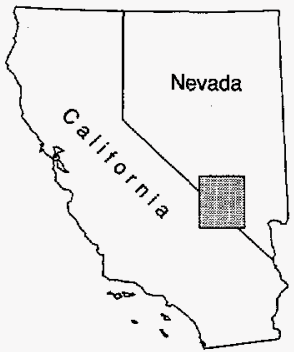

Figure 1. Study area with generalized well locations. 
The population in the area adjacent to Yucca Mountain increased substantially during the period of the water-level record shown in this report. The 1990 census (U.S. Bureau of the Census, 1990) reported a population of 1,623 individuals for the town of Beatty and 761 inhabitants in the town of Amargosa Valley (including the farm area south of the Amargosa Valley). The Yucca Flat division of Nye County had 1,016 persons, and the Crystal division had 92 persons. According to the 1990 census, the population of Nye County was 17,781 . In the 1980 census (U.S. Bureau of the Census, 1980), county divisions for statistical purposes were drawn differently. Most of the above-listed divisions were combined under one large "Beatty Township". This division included all of Nye County south of approximately latitude $37^{\circ} 30^{\prime} 00^{\prime \prime}$ N., except for about 7 percent of that area located in the extreme southeastern section known as the Pahrump Township division. Population figures from the 1980 census showed 3,524 persons in the Beatty Township and 9,048 persons in all of Nye County. The 1970 census (U.S. Bureau of the Census, 1970), using the same statistical divisions as those used in 1980, showed 1,131 persons in Beatty Township, and 5,599 in all of Nye County. The 1960 census (U.S. Bureau of the Census, 1960), which included the Pahrump area in Beatty Township, showed 1,153 persons in the Beatty division, and 4,374 individuals throughout the county.

Water consumption by local agriculture and industry in the general area was substantial although it varied during the period of water-level measurements. For example, Spring Meadows Ranch located in the Ash Meadows area pumped as much as 1.2 million cubic meters of water per month during 1970 and 1971 (Dudley and Larson, 1976) when a large, commercial cattle business was in operation.

Mining operations have been active in the study area with varying intensity since the early 1900 's. In the early 1980's, the American Borate Corporation processed its ores from Death Valley at the plant in the southern Amargosa Desert. Since that time, the company has scaled down production. Industrial Minerals Ventures processes bentonite in the same part of the Amargosa Desert. Gold mining near Beatty involved substantial withdrawals of ground water from 1989 to 1992.

The Nevada State Engineer estimated groundwater withdrawals in the Amargosa Valley basin at about 11.9 million cubic meters in 1985 . The estimate was about 5.1 million cubic meters in 1988 (R.J. LaCamera, U.S. Geological Survey, written commun., 1991). Operations at the Nevada Test Site involve pumping water from two wells in Jackass Flats. Estimated withdrawals from Jackass Flats on the Test Site were $194,100 \mathrm{~m}^{3}$ for 1985 and $174,100 \mathrm{~m}^{3}$ for 1988
(D.B. Wood, U.S. Geological Survey, written commun., 1991).

\section{REFERENCES CITED}

Czarnecki, J.B., and Waddell, R.K., 1984, Finite-element simulation of ground-water flow in the vicinity of Yucca Mountain, Nevada-California: U.S. Geological Survey Water-Resources Investigations Report 84-4349, 38 p. (NNA.870407.0173)

Dudley, W.,W., and Larson, J.D., 1976, Effect of irrigation pumping on Desert Pupfish habitats in Ash Meadows, Nye County, Nevada: U.S. Geological Survey Professional Paper 927, 26 p. (NNA.870518.0076)

Kilroy, K.C., 1991, Ground-water conditions in Amargosa Desert, Nevada-California, 1952-87: U.S. Geological Survey Water-Resources Investigations Report 89-4101, 93 p. (NNA.930602.0002)

McKinley, P.W., Long, M.P., and Benson, L.V., 1991, Chemical analyses of water from selected wells and springs in the Yucca Mountain area, Nevada and southeastern California: U.S. Geological Survey Open-File Report 90-355, 47 p. (NNA.901031.0004)

Nichols, W.D., and Akers, J.P., 1985, Water-level declines in the Amargosa Valley area, Nye County, Nevada, 1962-84: U.S. Geological Survey Water-Resources Investigations Report 85-4273, 7 p. (HQS.880517.1808)

Oatfield, W.J., and Czarnecki, J.B., 1989, Hydrogeologic inferences from drillers' logs and from gravity and resistivity surveys in the Amargosa Desert, southern Nevada: U.S. Geological Survey Open-File Report 89-234, 29 p. (NNA.901102.0005)

1990, 1990 Census of Population and Housing; Public Law 94-171 Data: U.S. Department of Commerce, Bureau of Census, Data User Services Division (CD-ROM), unnumbered pages. (MOL.19940919.0098)

1980, 1980 Census of Population; General Population Characteristics, Nevada: U.S. Department of Commerce, Bureau of Census, PC80-1-B30 Nevada, table 44. (MOL.19940919.0099)

1970, 1970 Census of Population, Nevada: U.S. Department of Commerce, Social and Economic Statistics Administration, Bureau of Census, v. 1, pt. 30, table 10. (MOL.19940919.0100)

1960, Census of Population: 1960, Characteristics of the Population, Nevada: U.S. Department of Commerce, Bureau of the Census: v. 1, pt. 30, table 7 and 8. (MOL.19940919.0101)

U.S. Geological Survey, 1986, Beatty Nevada-California: U.S. Geological Survey $30 \times 60$ minute topographic quadrangle, scale 1:100,000 metric. (NNA.930602.0011) 
U.S. Geological Survey, 1986, Death Valley Junction California-Nevada: U.S. Geological Survey $30 \times 60$ minute topographic quadrangle, scale 1:100,000 metric. (NNA.930602.0009) 1989, National water information system user's manual; volume 2, chapter 4 . Ground-water site inventory system: U.S. Geological Survey Open-File Report 89-587, 233 p. (NNA.930608.0008)

NOTE: Parenthesized numbers following each cited reference are for U.S. Department of Energy OCRWM Records Management purposes only and should not be used when ordering the publication. 
Table 1. Ground-water levels and well data of wells located in the Beatty, Nevada-California, 30-minute $\times 60$-minute quadrangle [“--", no data available; $m$, meter; cm, centimeter; Height of measurement point.--Minus sign indicates measuring point above land surface; NWIS, National Water Information System]

\begin{tabular}{|c|c|c|c|c|c|c|c|c|c|c|}
\hline & $\begin{array}{c}\text { Slte } \\
\text { number } \\
\text { (pl. 1) }\end{array}$ & Latitude & Longitude & $\begin{array}{l}\text { Depth } \\
\text { of well } \\
\text { below } \\
\text { land } \\
\text { surface } \\
\text { (m) }\end{array}$ & $\begin{array}{l}\text { Diameter of } \\
\text { casing } \\
\text { (cm) }\end{array}$ & $\begin{array}{l}\text { Helght of } \\
\text { measuring } \\
\text { point above } \\
\text { land surface } \\
\text { (m) }\end{array}$ & $\begin{array}{l}\text { Water } \\
\text { level } \\
\text { below } \\
\text { land } \\
\text { surface } \\
\text { (m) }\end{array}$ & $\begin{array}{l}\text { Water } \\
\text { level } \\
\text { altitude } \\
\text { (m) }\end{array}$ & $\begin{array}{l}\text { Date of } \\
\text { measurement }\end{array}$ & NWIS Site-ID \\
\hline & 11 & $37^{\circ} 00^{\prime} 53^{\prime \prime}$ & $116^{\circ} 00^{\prime} 29^{\prime \prime}$ & 491 & 274.32 & -- & 472 & 737.0 & $10-07-77$ & 370053116002901 \\
\hline & $1_{2}$ & $37^{\circ} 00^{\prime} 20^{\prime \prime}$ & $116^{\circ} 00^{\prime} 37^{\prime \prime}$ & 488 & 274.32 & 0.0 & 467 & 739.0 & $01-28-91$ & 370020116003701 \\
\hline & $1_{3}$ & $37^{\circ} 00^{\prime} 20^{\prime \prime}$ & $115^{\circ} 59^{\prime} 30^{\prime \prime}$ & 546 & 375.92 & 0.0 & 480 & 740.0 & $04-17-91$ & 370020115593001 \\
\hline & 14 & $37^{\circ} 00^{\prime} 17^{\prime \prime}$ & $116^{\circ} 01^{\prime} 42^{\prime \prime}$ & 503 & 167.64 & - & 472 & 735.0 & 09-09-72 & 370017116014201 \\
\hline & ${ }^{15}$ & $37^{\circ} 00^{\prime} 05^{\prime \prime}$ & $116^{\circ} 04^{\prime} 03^{\prime \prime}$ & 985 & 34.04 & 0.6 & 474 & 744.0 & $11-05-90$ & 370005116040301 \\
\hline & 6 & $36^{\circ} 59^{\prime} 43^{\prime \prime}$ & $116^{\circ} 03^{\prime} 29^{\prime \prime}$ & 536 & 20.32 & -- & 470 & 740.0 & $01-10-80$ & 365943116032901 \\
\hline & 7 & $36^{\circ} 59^{\prime} 05^{\prime \prime}$ & $116^{\circ} 03^{\prime} 32^{\prime \prime}$ & 1188 & 34.04 & -- & 462 & 741.0 & $01-28-91$ & 365905116033201 \\
\hline & 8 & $36^{\circ} 59^{\prime} 05^{\prime \prime}$ & $116^{\circ} 01^{\prime} 20^{\prime \prime}$ & 1283 & 50.80 & -- & 460 & 740.0 & $11-05-90$ & 365905116012001 \\
\hline & 9 & $36^{\circ} 58^{\prime} 45^{\prime \prime}$ & $116^{\circ} 00^{\prime} 49^{\prime \prime}$ & 509 & 32.25 & -- & 459 & 739.0 & $10-22-71$ & 365848116000801 \\
\hline & 10 & $36^{\circ} 58^{\prime} 45^{\prime \prime}$ & $116^{\circ} 00^{\prime} 49^{\prime \prime}$ & 509 & 32.25 & -0.36 & 458 & 740.0 & $03-06-91$ & 365849116002101 \\
\hline & 11 & $36^{\circ} 56^{\prime} 29^{\prime \prime}$ & $116^{\circ} 22^{\prime} 26^{\prime \prime}$ & 355 & 77.47 & -- & 27.59 & 1187.4 & $02-19-84$ & 365629116222602 \\
\hline & 12 & $36^{\circ} 56^{\prime} 24^{\prime \prime}$ & $116^{\circ} 22^{\prime} 29^{\prime \prime}$ & -- & 13.97 & -- & 16.3 & 1186.7 & $01-21-86$ & 365624116222901 \\
\hline & 13 & $36^{\circ} 55^{\prime} 50^{\prime \prime}$ & $116^{\circ} 09^{\prime} 11^{\prime \prime}$ & 1122 & 76.20 & 1.95 & 508 & 819.0 & $11-06-90$ & 365550116091101 \\
\hline & 14 & $36^{\circ} 55^{\prime} 50^{\prime \prime}$ & $116^{\circ} 08^{\prime} 42^{\prime \prime}$ & 1006 & 50.80 & -- & 502 & 821.0 & $09-23-83$ & 365550116084201 \\
\hline & 15 & $36^{\circ} 55^{\prime} 24^{\prime \prime}$ & $116^{\circ} 44^{\prime} 40^{\prime \prime}$ & 61 & -- & -- & 28.96 & 1068.0 & -- & 365524116444001 \\
\hline \multirow{8}{*}{ 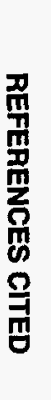 } & 16 & $36^{\circ} 55^{\prime} 20^{\prime \prime}$ & $116^{\circ} 37^{\prime} 03^{\prime \prime}$ & 488 & -- & -0.1 & 203 & 993.0 & $09-11-90$ & 365520116370301 \\
\hline & 17 & $36^{\circ} 55^{\prime} 17^{\prime \prime}$ & $116^{\circ} 36^{\prime} 58^{\prime \prime}$ & 488 & - & -- & 203 & 990.0 & $09-11-90$ & 365517116365801 \\
\hline & 18 & $36^{\circ} 55^{\prime} 08^{\prime \prime}$ & $116^{\circ} 00^{\prime} 35^{\prime \prime}$ & 518 & 32.25 & - & 471 & 724.0 & $10-18-75$ & 365508116003501 \\
\hline & 19 & $36^{\circ} 55^{\prime} 07^{\prime \prime}$ & $116^{\circ} 00^{\prime} 35^{\prime \prime}$ & 503 & 60.96 & -- & 471 & 724.0 & $08-06-72$ & 365500116003901 \\
\hline & 20 & $36^{\circ} 54^{\prime} 57^{\prime \prime}$ & $116^{\circ} 51^{\prime} 58^{\prime \prime}$ & 53 & -- & -- & 24.38 & 1316.6 & $\ldots$ & 365457116515801 \\
\hline & 21 & $36^{\circ} 54^{\prime} 45^{\prime \prime}$ & $116^{\circ} 38^{\prime} 39^{\prime \prime}$ & 213 & 16.84 & -0.5 & 44.9 & 1199.1 & $09-11-90$ & 365445116383901 \\
\hline & 22 & $36^{\circ} 54^{\prime} 37^{\prime \prime}$ & $116^{\circ} 38^{\prime} 44^{\prime \prime}$ & $-\cdot$ & -- & $\cdots$ & 45 & 1174.0 & $09-11-90$ & 365437116384401 \\
\hline & 23 & $36^{\circ} 54^{\prime} 18^{\prime \prime}$ & $116^{\circ} 01 ' 26^{\prime \prime}$ & 439 & 77.72 & - & 260 & 838.0 & $03-18-91$ & 365418116012601 \\
\hline
\end{tabular}


Table 1. Ground-water levels and well data of wells located in the Beatty, Nevada-California, 30-minute $\times 60$-minute quadrangle--Continued

\begin{tabular}{|c|c|c|c|c|c|c|c|c|c|}
\hline $\begin{array}{c}\text { Site } \\
\text { number } \\
\text { (pl. 1) }\end{array}$ & Latitude & Longitude & $\begin{array}{l}\text { Depth } \\
\text { of well } \\
\text { below } \\
\text { land } \\
\text { surface } \\
\text { (m) }\end{array}$ & $\begin{array}{l}\text { Diameter of } \\
\text { casing } \\
\text { (cm) }\end{array}$ & $\begin{array}{c}\text { Height of } \\
\text { measuring } \\
\text { point above } \\
\text { land surface } \\
\text { (m) }\end{array}$ & $\begin{array}{l}\text { Water } \\
\text { lovel } \\
\text { below } \\
\text { land } \\
\text { surface } \\
\text { (m) }\end{array}$ & $\begin{array}{l}\text { Water } \\
\text { level } \\
\text { aititude } \\
\text { (m) }\end{array}$ & $\begin{array}{c}\text { Date of } \\
\text { measurement }\end{array}$ & NWIS SIto-1D \\
\hline 24 & $36^{\circ} 54^{\prime} 12^{\prime \prime}$ & $116^{\circ} 01^{\prime} 39^{\prime \prime}$ & 458 & 91.44 & 1.1 & 255 & 844.0 & $03-18-91$ & 365412116013901 \\
\hline 25 & $36^{\circ} 54^{\prime} 09^{\prime \prime}$ & $116^{\circ} 45^{\prime} 23^{\prime \prime}$ & 59 & -- & -- & 6.10 & 1151.9 & -- & 365409116452301 \\
\hline 26 & $36^{\circ} 53^{\prime} 40^{\prime \prime}$ & $116^{\circ} 26^{\prime} 46^{\prime \prime}$ & 372 & 27.17 & -- & 281 & 1034.0 & $07-21-86$ & 365340116264601 \\
\hline 27 & $36^{\circ} 53^{\prime 2} 26^{\prime \prime}$ & $116^{\circ} 52^{\prime} 02^{\prime \prime}$ & 122 & 20.32 & -0.5 & 83.1 & 1080.9 & $01-06-87$ & 365326116520201 \\
\hline 28 & $36^{\circ} 53^{\prime} 22^{\prime \prime}$ & $116^{\circ} 27^{\prime} 35^{\prime \prime}$ & 1831 & 34.04 & -- & 525 & 1029.0 & $09-17-82$ & 365322116273501 \\
\hline 29 & $36^{\circ} 52^{\prime} 47^{\prime \prime}$ & $116^{\circ} 45^{\prime} 18^{\prime \prime}$ & 38 & 25.4 & -0.2 & 5.37 & 963.6 & $01-15-87$ & 365247116451801 \\
\hline 30 & $36^{\circ} 52^{\prime} 39^{\prime \prime}$ & $116^{\circ} 25^{\prime} 34^{\prime \prime}$ & 510 & 27.17 & -- & 472 & 739.0 & $08-06-86$ & 365239116253401 \\
\hline 31 & $36^{\circ} 52^{\prime} 39^{\prime \prime}$ & $116^{\circ} 02^{\prime} 09^{\prime \prime}$ & -- & -- & -. & 243 & 848.0 & $03-29-66$ & 365239116020901 \\
\hline 32 & $36^{\circ} 52^{\prime} 00^{\prime \prime}$ & $116^{\circ} 27^{\prime} 29^{\prime \prime}$ & -- & 52.07 & -- & 571.7 & 754.3 & $03-23-82$ & 365200116272901 \\
\hline 33 & $36^{\circ} 51^{\prime} 57^{\prime \prime}$ & $116^{\circ} 27^{\prime} 12^{\prime \prime}$ & -- & 77.72 & -- & 572 & 731.0 & $06-24-82$ & 365157116271201 \\
\hline 34 & $36^{\circ} 51^{\prime} 57^{\prime \prime}$ & $116^{\circ} 27^{\prime} 12^{\prime \prime}$ & -- & -- & -- & 518 & 785.0 & $11-16-84$ & 365157116271202 \\
\hline 35 & $36^{\circ} 51^{\prime} 57^{\prime \prime}$ & $116^{\circ} 27^{\prime} 12^{\prime \prime}$ & -- & - & -- & 567 & 736.0 & 08-26-85 & 365157116271203 \\
\hline 36 & $36^{\circ} 51^{\prime} 57^{\prime \prime}$ & $116^{\circ} 27^{\prime} 12^{\prime \prime}$ & -- &.- & -. & 573 & 730.0 & $12-01-84$ & 365157116271204 \\
\hline 37 & $36^{\circ} 51^{\prime} 57^{\prime \prime}$ & $116^{\circ} 27^{\prime} 12^{\prime \prime}$ & -- & -. & -- & 573 & 730.0 & $12-13-84$ & 365157116271205 \\
\hline 38 & $36^{\circ} 51^{\prime} 40^{\prime \prime}$ & $116^{\circ} 26^{\prime} 03^{\prime \prime}$ & 478 & 27.17 & -- & 438 & 731.0 & $12-23-87$ & 365140116260301 \\
\hline 39 & $36^{\circ} 51^{\prime} 22^{\prime \prime}$ & $116^{\circ} 27^{\prime} 55^{\prime \prime}$ & -- & 75.95 & - & 704 & 775.0 & $12-22-82$ & 365122116275501 \\
\hline 40 & $36^{\circ} 51^{\prime 22}$ & $116^{\circ} 27^{\prime} 55^{\prime \prime}$ & -- & -- & -- & 704 & 775.0 & $02-22-85$ & 365122116275502 \\
\hline 41 & $36^{\circ} 51^{\prime} 22^{\prime \prime}$ & $116^{\circ} 27^{\prime} 55^{\prime \prime}$ & - & -- & -- & 704 & 775.0 & $02-22-85$ & 365122116275503 \\
\hline 42 & $36^{\circ} 51^{\prime} 16^{\prime \prime}$ & $116^{\circ} 23^{\prime} 38^{\prime \prime}$ & 407 & 27.17 & - & 354 & 729.0 & $12-28-87$ & 365116116233801 \\
\hline 43 & $36^{\circ} 51^{\prime} 14^{\prime \prime}$ & $116^{\circ} 27^{\prime} 04^{\prime \prime}$ & -- & 34.04 & - & 539 & 730.0 & $07-23-86$ & 365114116270401 \\
\hline 44 & $36^{\circ} 51^{\prime} 08^{\prime \prime}$ & $116^{\circ} 26^{\prime} 23^{\prime \prime}$ & -- & 52.07 & -- & 471 & 730.0 & $12-30-82$ & 365108116262301 \\
\hline 45 & $36^{\circ} 51^{\prime} 08^{\prime \prime}$ & $116^{\circ} 26^{\prime} 23^{\prime \prime}$ & - & -- & -- & 472 & 729.0 & $09-13-83$ & 365108116262302 \\
\hline 46 & $36^{\circ} 51^{\prime} 08^{\prime \prime}$ & $116^{\circ} 26^{\prime} 23^{\prime \prime}$ & -- & -- & -- & 470 & 731.0 & $08-01-83$ & 365108116262303 \\
\hline 47 & $36^{\circ} 51^{\prime} 06^{\prime \prime}$ & $116^{\circ} 49^{\prime} 32^{\prime \prime}$ & 364 & -- & -- & 219 & -- & $08-10-88$ & 365106116493200 \\
\hline
\end{tabular}


Table 1. Ground-water levels and well data of wells located in the Beatty, Nevada-California, 30-minute $\times 60$-minute quadrangle--Continued

\begin{tabular}{|c|c|c|c|c|c|c|c|c|c|}
\hline $\begin{array}{c}\text { Site } \\
\text { number } \\
\text { (pl. 1) }\end{array}$ & Latitude & Longitude & $\begin{array}{l}\text { Depth } \\
\text { of well } \\
\text { below } \\
\text { land } \\
\text { surface } \\
\text { (m) }\end{array}$ & $\begin{array}{l}\text { Diameter of } \\
\text { casing } \\
\text { (cm) }\end{array}$ & $\begin{array}{c}\text { Height of } \\
\text { measuring } \\
\text { point above } \\
\text { land surface } \\
(m)\end{array}$ & $\begin{array}{c}\text { Water } \\
\text { lovel } \\
\text { below } \\
\text { land } \\
\text { surface } \\
\text { (m) }\end{array}$ & $\begin{array}{l}\text { Water } \\
\text { level } \\
\text { altitude } \\
\text { (m) }\end{array}$ & $\begin{array}{c}\text { Date of } \\
\text { measurement }\end{array}$ & NWIS SIte-1D \\
\hline 48 & $36^{\circ} 51^{\prime} 05^{\prime \prime}$ & $116^{\circ} 26^{\prime} 24^{\prime \prime}$ & $\cdots$ & 34.04 & -- & 468 & 731.0 & $04-29-85$ & 365105116262401 \\
\hline 49 & $36^{\circ} 50^{\prime} 49^{\prime \prime}$ & $116^{\circ} 28^{\prime} 55^{\prime \prime}$ & -- & 76.20 & -- & 527 & 775.0 & $12-15-82$ & 365049116285501 \\
\hline 50 & $36^{\circ} 50^{\prime} 49^{\prime \prime}$ & $116^{\circ} 28^{\prime} 55^{\prime \prime}$ & -- & -. & -- & 526 & 776.0 & $05-23-84$ & 365049116285502 \\
\hline 51 & $36^{\circ} 50^{\prime} 49^{\prime \prime}$ & $116^{\circ} 28^{\prime} 55^{\prime \prime}$ & -- & - & -- & 524 & 778.0 & $05-23-84$ & 365049116285503 \\
\hline 52 & $36^{\circ} 50^{\prime} 49^{\prime \prime}$ & $116^{\circ} 28^{\prime} 55^{\prime \prime}$ & -- & -- & -- & 526 & 776.0 & $08-13-85$ & 365049116285504 \\
\hline 53 & $36^{\circ} 50^{\prime} 49^{\prime \prime}$ & $116^{\circ} 28^{\prime} 55^{\prime \prime}$ & -- & -- & -- & 526 & 776.0 & $08-12-84$ & 365049116285505 \\
\hline 54 & $36^{\circ} 50^{\prime} 32^{\prime \prime}$ & $116^{\circ} 26^{\prime} 54^{\prime \prime}$ & -. & 76.20 & -- & 519 & 729.0 & $12-30-82$ & 365032116265401 \\
\hline 55 & $36^{\circ} 50^{\prime} 32^{\prime \prime}$ & $116^{\circ} 26^{\prime} 54^{\prime \prime}$ & -. & -- & -- & 519 & 729.0 & $02-14-85$ & 365032116265402 \\
\hline 56 & $36^{\circ} 50^{\prime} 32^{\prime \prime}$ & $116^{\circ} 26^{\prime} 54^{\prime \prime}$ & -. & -- & -- & 518 & 730.0 & $02-14-85$ & 365032116265403 \\
\hline 57 & $36^{\circ} 50^{\prime} 32^{\prime \prime}$ & $116^{\circ} 24^{\prime} 35^{\prime \prime}$ & 397 & 27.17 & -- & 346 & 730.0 & $12-28-87$ & 365032116243501 \\
\hline 58 & $36^{\circ} 50^{\prime} 23^{\prime \prime}$ & $116^{\circ} 27^{\prime} 18^{\prime \prime}$ & 622 & 27.17 & -. & 571 & 730.0 & $03-20-85$ & 365023116271801 \\
\hline 59 & $36^{\circ} 49^{\prime} 47^{\prime \prime}$ & $116^{\circ} 25^{\prime} 43^{\prime \prime}$ & 903 & 76.20 & .- & 400.3 & 730.7 & $11-07-83$ & 364947116254300 \\
\hline 60 & $36^{\circ} 49^{\prime} 47^{\prime \prime}$ & $116^{\circ} 25^{\prime} 43^{\prime \prime}$ & .- & 76.20 & -- & 402.5 & 729.5 & $05-02-84$ & 364947116254302 \\
\hline 62 & $36^{\circ} 49^{\prime} 45^{\prime \prime}$ & $116^{\circ} 25^{\prime} 43^{\prime \prime}$ & -- & 76.20 & -- & 402.5 & 729.5 & $03-18-84$ & 364945116254301 \\
\hline 63 & $36^{\circ} 49^{\prime} 45^{\prime \prime}$ & $116^{\circ} 23^{\prime} 50^{\prime \prime}$ & 346 & 27.17 & -. & 304 & 728.0 & $02-12-85$ & 364945116235001 \\
\hline 64 & $36^{\circ} 49^{\prime} 42^{\prime \prime}$ & $116^{\circ} 28^{\prime} 00^{\prime \prime}$ & -. & 78.74 & -- & 752 & 731.0 & $05-18-84$ & 364942116280001 \\
\hline 65 & $36^{\circ} 49^{\prime} 42^{\prime \prime}$ & $116^{\circ} 28^{\prime} 00^{\prime \prime}$ & -- & - & -- & 751 & 732.0 & $11-21-83$ & 364942116280002 \\
\hline 66 & $36^{\circ} 49^{\prime} 42^{\prime \prime}$ & $116^{\circ} 28^{\prime} 00^{\prime \prime}$ & -. & $\ldots$ & -- & 729 & 754.0 & $11-21-83$ & 364942116280003 \\
\hline 67 & $36^{\circ} 49^{\prime} 42^{\prime \prime}$ & $116^{\circ} 28^{\prime} 00^{\prime \prime}$ & .- & -- & -. & 752 & 731.0 & $10-15-85$ & 364942116280004 \\
\hline 68 & $36^{\circ} 49^{\prime} 42^{\prime \prime}$ & $116^{\circ} 28^{\prime} 00^{\prime \prime}$ & -- & -- & .- & 710 & 773.0 & $10-15-85$ & 364942116280005 \\
\hline 69 & $36^{\circ} 49^{\prime} 38^{\prime \prime}$ & $116^{\circ} 25^{\prime} 21^{\prime \prime}$ & -. & 60.96 & -. & 364.7 & 749.3 & $11-07-83$ & 364938116252101 \\
\hline 71 & $36^{\circ} 49^{\prime} 38^{\prime \prime}$ & $116^{\circ} 25^{\prime} 21^{\prime \prime}$ & -- & -- & -- & 362 & 752.0 & $10-12-84$ & 364938116252102 \\
\hline
\end{tabular}


Table 1. Ground-water levels and well data of wells located in the Beatty, Nevada-California, 30-minute $\times 60-$ minute quadrangle--Continued

\begin{tabular}{|c|c|c|c|c|c|c|c|c|c|}
\hline $\begin{array}{l}\text { Site } \\
\text { number } \\
\text { (pl. 1) }\end{array}$ & Latitude & Longitude & $\begin{array}{l}\text { Depth } \\
\text { of well } \\
\text { below } \\
\text { land } \\
\text { surface } \\
\text { (m) }\end{array}$ & $\begin{array}{l}\text { Diameter of } \\
\text { casing } \\
\text { (cm) }\end{array}$ & $\begin{array}{c}\text { Height of } \\
\text { measuring } \\
\text { point above } \\
\text { land surface } \\
\text { (m) }\end{array}$ & $\begin{array}{l}\text { Water } \\
\text { level } \\
\text { below } \\
\text { land } \\
\text { surface } \\
\text { (m) }\end{array}$ & $\begin{array}{l}\text { Water } \\
\text { level } \\
\text { altitude } \\
\text { (m) }\end{array}$ & $\begin{array}{l}\text { Date of } \\
\text { measurement }\end{array}$ & NWIS Site-ID \\
\hline 72 & $36^{\circ} 49^{\prime} 35^{\prime \prime}$ & $116^{\circ} 06^{\prime} 54^{\prime \prime}$ & - & 11.43 & -- & 33.4 & 1232.6 & $07-21-62$ & 364935116065401 \\
\hline 73 & $36^{\circ} 49^{\prime} 33^{\prime \prime}$ & $116^{\circ} 28^{\prime} 57^{\prime \prime}$ & 481 & 27.17 & -- & 421 & 776.0 & $05-27-87$ & 364933116285701 \\
\hline 74 & $36^{\circ} 49^{\prime} 20^{\prime \prime}$ & $116^{\circ} 06^{\prime} 42^{\prime \prime}$ & -- & -- & -- & 50.7 & 1196.3 & $07-21-62$ & 364920116064201 \\
\hline 75 & $36^{\circ} 49^{\prime} 16^{\prime \prime}$ & $116^{\circ} 26^{\prime} 56^{\prime \prime}$ & 507 & 27.17 & -- & 471 & 730.0 & $12-23-87$ & 364916116265601 \\
\hline 76 & $36^{\circ} 49^{\prime} 05^{\prime \prime}$ & $116^{\circ} 28^{\prime} 01^{\prime \prime}$ & -- & 34.04 & -- & 750 & 730.0 & $03-13-86$ & 364905116280101 \\
\hline 77 & $36^{\circ} 48^{\prime} 37^{\prime \prime}$ & $116^{\circ} 34^{\prime} 52^{\prime \prime}$ & .- & 15.24 & -0.79 & 141 & 837.0 & $03-10-85$ & 364837116345201 \\
\hline 78 & $36^{\circ} 48^{\prime} 29^{\prime \prime}$ & $116^{\circ} 23^{\prime} 40^{\prime \prime}$ & 1064 & 76.20 & -- & 283 & 728.0 & $09-11-86$ & 364828116234001 \\
\hline 80 & $36^{\circ} 48^{\prime} 25^{\prime \prime}$ & $116^{\circ} 29^{\prime} 05^{\prime \prime}$ & 403 & 27.17 & -- & 347 & 776.0 & $12-16-87$ & 364825116290501 \\
\hline 81 & $36^{\circ} 48^{\prime} 22^{\prime \prime}$ & $116^{\circ} 26^{\prime} 26^{\prime \prime}$ & 419 & 27.17 & - & 394 & 730.0 & $12-23-87$ & 364822116262601 \\
\hline 82 & $36^{\circ} 48^{\prime} 14^{\prime \prime}$ & $116^{\circ} 48^{\prime} 54^{\prime \prime}$ & 457 & 7.62 & -0.6 & 166 & 791.0 & $01-11-87$ & 364814116485401 \\
\hline 84 & $36^{\circ} 47^{\prime} 57^{\prime \prime}$ & $116^{\circ} 24^{\prime} 58^{\prime \prime}$ & 343 & 40.64 & -- & 301 & 729.0 & $03-22-85$ & 364757116245801 \\
\hline 85 & $36^{\circ} 47^{\prime} 32^{\prime \prime}$ & $116^{\circ} 33^{\prime} 07^{\prime \prime}$ & - & 24.43 & -- & 184 & 779.0 & $12-16-87$ & 364732116330701 \\
\hline 86 & $36^{\circ} 47^{\prime} 06^{\prime \prime}$ & $116^{\circ} 17^{\prime} 06^{\prime \prime}$ & -- & 32.25 & - & 317 & 733.0 & $12-19-64$ & 364706116170601 \\
\hline 87 & $36^{\circ} 46^{\prime} 56^{\prime \prime}$ & $116^{\circ} 26^{\prime} 16^{\prime \prime}$ & 385 & 27.17 & -- & 345 & 730.0 & $12-28-87$ & 364656116261601 \\
\hline 88 & $36^{\circ} 46^{\prime} 49^{\prime \prime}$ & $116^{\circ} 28^{\prime} 02^{\prime \prime}$ & 416 & 27.17 & -- & 364 & 730.0 & $10-15-86$ & 364649116280201 \\
\hline 89 & $36^{\circ} 46^{\prime} 22^{\prime \prime}$ & $116^{\circ} 16^{\prime} 43^{\prime \prime}$ & 405 & 32.25 & 0.91 & 318 & 731.0 & 09-13-57 & 364622116164301 \\
\hline 90 & $36^{\circ} 46^{\prime} 15^{\prime \prime}$ & $116^{\circ} 41^{\prime \prime} 24^{\prime \prime}$ & 94 & 15.24 & -0.5 & 87.3 & 761.7 & $05-15-89$ & 364615116412401 \\
\hline 91 & $36^{\circ} 46^{\prime} 07^{\prime \prime}$ & $116^{\circ} 41^{\prime} 07^{\prime \prime}$ & 91 & 15.24 & -0.5 & 86.5 & 759.5 & $05-15-89$ & 364607116410701 \\
\hline 92 & $36^{\circ} 46^{\prime} 03^{\prime \prime}$ & $116^{\circ} 41^{\prime} 21^{\prime \prime}$ & 92 & 15.24 & -0.5 & 86.7 & 760.3 & $05-15-89$ & 364603116412101 \\
\hline 94 & $36^{\circ} 46^{\prime} 00^{\prime \prime}$ & $116^{\circ} 41^{\prime} 20^{\prime \prime}$ & 98 & 15.24 & -0.5 & 92.0 & 754.0 & $05-15-89$ & 364600116412001 \\
\hline 95 & $36^{\circ} 46^{\prime} 00^{\prime \prime}$ & $116^{\circ} 41^{\prime} 09^{\prime \prime}$ & 99 & 15.24 & -0.5 & 92.3 & 752.7 & $05-15-89$ & 364600116410901 \\
\hline 96 & $36^{\circ} 45^{\prime} 59^{\prime \prime}$ & $116^{\circ} 52^{\prime} 19^{\prime \prime}$ & 145 & 15.24 & -0.2 & 145 & 1001.0 & $10-30-86$ & 364559116521901 \\
\hline
\end{tabular}


Table 1. Ground-water levels and well data of wells located in the Beatty, Nevada-California, 30-minute $\times 60$-minute quadrangle--Continued

\begin{tabular}{|c|c|c|c|c|c|c|c|c|c|}
\hline $\begin{array}{c}\text { Site } \\
\text { number } \\
\text { (pl. 1) }\end{array}$ & Latitude & Longitude & $\begin{array}{l}\text { Depth } \\
\text { of well } \\
\text { below } \\
\text { land } \\
\text { surface } \\
\text { (m) }\end{array}$ & $\begin{array}{l}\text { Diameter of } \\
\text { casing } \\
\text { (cm) }\end{array}$ & $\begin{array}{l}\text { Height of } \\
\text { measuring } \\
\text { point above } \\
\text { land surface } \\
\text { (m) }\end{array}$ & $\begin{array}{l}\text { Water } \\
\text { level } \\
\text { below } \\
\text { land } \\
\text { surface } \\
\text { (m) }\end{array}$ & $\begin{array}{l}\text { Water } \\
\text { level } \\
\text { altitude } \\
(\mathbf{m})\end{array}$ & $\begin{array}{c}\text { Date of } \\
\text { measurement }\end{array}$ & NWIS SIte-ID \\
\hline 97 & $36^{\circ} 45^{\prime} 57^{\prime \prime}$ & $116^{\circ} 43^{\prime} 45^{\prime \prime}$ & 123 & 7.62 & -- & 103 & 757.0 & $08-21-83$ & 364557116434501 \\
\hline 98 & $36^{\circ} 45^{\prime} 57^{\prime \prime}$ & $116^{\circ} 41^{\prime} 30^{\prime \prime}$ & 175 & 20.32 & -0.2 & 96.0 & 751.5 & $06-27-61$ & 364600116413001 \\
\hline 99 & $36^{\circ} 45^{\prime} 57^{\prime \prime}$ & $116^{\circ} 41^{\prime} 18^{\prime \prime}$ & 99 & 15.24 & -0.5 & 92.8 & 752.2 & 05-15-89 & 364557116411801 \\
\hline 100 & $36^{\circ} 45^{\prime} 57^{\prime \prime}$ & $116^{\circ} 41^{\prime} 14^{\prime \prime}$ & 99 & 15.24 & -0.6 & 92.5 & 751.5 & $05-15-89$ & 364557116411401 \\
\hline 101 & $36^{\circ} 45^{\prime} 57^{\prime \prime}$ & $116^{\circ} 41^{\prime} 12^{\prime \prime}$ & 175 & 20.32 & .15 & 85.9 & 764.1 & $07-12-62$ & 364557116411201 \\
\hline 102 & $36^{\circ} 45^{\prime} 57^{\prime \prime}$ & $116^{\circ} 41^{\prime} 11^{\prime \prime}$ & 99 & 15.24 & -0.6 & 93.1 & 750.9 & 05-15-89 & 364557116411101 \\
\hline 103 & $36^{\circ} 45^{\prime} 57^{\prime \prime}$ & $116^{\circ} 41^{\prime} 09^{\prime \prime}$ & 99 & 15.24 & -0.6 & 93.1 & 751.9 & $05-15-89$ & 364557116410901 \\
\hline 104 & $36^{\circ} 45^{\prime} 56^{\prime \prime}$ & $116^{\circ} 41^{\prime} 35^{\prime \prime}$ & 123 & 15.24 & -0.3 & 112 & 734.0 & 03-06-91 & 364556116413501 \\
\hline 105 & $36^{\circ} 45^{\prime} 54^{\prime \prime}$ & $116^{\circ} 48^{\prime} 55^{\prime \prime}$ & 98 & -- & 0.0 & 74.5 & 913.5 & $01-11-87$ & 364554116485501 \\
\hline 106 & $36^{\circ} 45 ' 54^{\prime \prime}$ & $116^{\circ} 23^{\prime} 24^{\prime \prime}$ & 347 & 32.25 & -- & 226.2 & 726.8 & $12-05-83$ & 364554116232401 \\
\hline 107 & $36^{\circ} 45^{\prime} 52^{\prime \prime}$ & $116^{\circ} 41^{\prime} 33^{\prime \prime}$ & 126 & - & -- & 109 & 738.0 & $02-27-85$ & 364552116413301 \\
\hline 108 & $36^{\circ} 45^{\prime} 50^{\prime \prime}$ & $116^{\circ} 47^{\prime} 27^{\prime \prime}$ & 143 & -- & 0.0 & 127 & 800.0 & $01-11-87$ & 364550116472701 \\
\hline 109 & $36^{\circ} 45^{\prime} 39^{\prime \prime}$ & $116^{\circ} 02^{\prime} 43^{\prime \prime}$ & -- & 50.80 & -. & 158 & 909.0 & $04-00-66$ & 364539116024301 \\
\hline 110 & $36^{\circ} 45^{\prime} 34^{\prime \prime}$ & $116^{\circ} 06^{\prime} 59^{\prime \prime}$ & 1036 & 32.25 & -0.62 & 529 & 734.0 & $10-26-89$ & 364534116065901 \\
\hline 111 & $36^{\circ} 45^{\prime} 34^{\prime \prime}$ & $116^{\circ} 06^{\prime} 59^{\prime \prime}$ & -- & 45.72 & -0.50 & 529 & -- & 05-02-90 & 364534116065902 \\
\hline 112 & $36^{\circ} 45^{\prime} 14^{\prime \prime}$ & $116^{\circ} 48^{\prime} 29^{\prime \prime}$ & 116 & -- & -0.7 & 108 & 873.0 & 01-01-87 & 364514116482901 \\
\hline 113 & $36^{\circ} 45^{\prime} 08^{\prime \prime}$ & $116^{\circ} 25^{\prime} 54^{\prime \prime}$ & 270 & 32.25 & -0.91 & 224 & 729.0 & $10-31-57$ & 364457116231201 \\
\hline 114 & $36^{\circ} 42^{\prime} 47^{\prime \prime}$ & $116^{\circ} 44^{\prime} 23^{\prime \prime}$ & 579 & 5.08 & -0.3 & 92.4 & 736.6 & $01-11-87$ & 364247116442301 \\
\hline 115 & $36^{\circ} 42^{\prime} 46^{\prime \prime}$ & $116^{\circ} 44^{\prime} 57^{\prime \prime}$ & 427 & -- & 0.0 & 85.8 & 743.2 & $01-11-87$ & 364246116445701 \\
\hline 116 & $36^{\circ} 42^{\prime} 43^{\prime \prime}$ & $116^{\circ} 43^{\prime} 24^{\prime \prime}$ & 567 & 5.08 & -0.8 & 92.8 & 730.2 & $01-11-87$ & 364243116432401 \\
\hline 117 & $36^{\circ} 42^{\prime} 40^{\prime \prime}$ & $116^{\circ} 36^{\prime} 53^{\prime \prime}$ & 139 & -- & -- & 71.8 & 716.2 & $05-11-90$ & 364215116362701 \\
\hline 118 & $36^{\circ} 41^{\prime} 30^{\prime \prime}$ & $116^{\circ} 41^{\prime} 12^{\prime \prime}$ & 279 & 5.08 & -0.5 & 82.1 & 716.9 & $01-11-87$ & 364141116351401 \\
\hline 119 & $36^{\circ} 41^{\prime} 30^{\prime \prime}$ & $116^{\circ} 41^{\prime} 12^{\prime \prime}$ & 98 & 5.08 & -0.5 & 82.1 & 722.9 & $01-11-87$ & 364141116351402 \\
\hline 120 & $36^{\circ} 41^{\prime} 09^{\prime \prime}$ & $116^{\circ} 46^{\prime} 59^{\prime \prime}$ & 341 & -- & -- & 250 & 753.0 & $01-30-91$ & 364111116470101 \\
\hline
\end{tabular}




\begin{tabular}{|c|c|c|c|c|c|c|c|c|c|}
\hline $\begin{array}{c}\text { Site } \\
\text { number. } \\
\text { (pl. 1) }\end{array}$ & Latitude & Longltude & $\begin{array}{l}\text { Depth } \\
\text { of well } \\
\text { below } \\
\text { land } \\
\text { surface } \\
\text { (m) }\end{array}$ & $\begin{array}{l}\text { Diameter of } \\
\text { casing } \\
\text { (cm) }\end{array}$ & $\begin{array}{l}\text { Height of } \\
\text { measuring } \\
\text { point above } \\
\text { land surface } \\
\text { (m) }\end{array}$ & $\begin{array}{c}\text { Water } \\
\text { lovel } \\
\text { below } \\
\text { land } \\
\text { surface } \\
\text { (m) }\end{array}$ & $\begin{array}{c}\text { Water } \\
\text { level } \\
\text { altitude } \\
(m)\end{array}$ & $\begin{array}{c}\text { Date of } \\
\text { measurement }\end{array}$ & NWIS Site-ID \\
\hline 121 & $36^{\circ} 39^{\prime} 07^{\prime \prime}$ & $116^{\circ} 23^{\prime} 57^{\prime \prime}$ & 144 & 12.00 & -- & 101 & 719.0 & $05-20-61$ & 363907116235701 \\
\hline 122 & $36^{\circ} 38^{\prime} 40^{\prime \prime}$ & $116^{\circ} 23^{\prime} 50^{\prime \prime}$ & 154 & 25.40 & -- & 110 & 704.0 & $02-28-55$ & 363840116235000 \\
\hline 123 & $36^{\circ} 38^{\prime} 40^{\prime \prime}$ & $116^{\circ} 23^{\prime} 40^{\prime \prime}$ & 120 & 20.32 & -. & 105 & 706.0 & $05-03-52$ & 363840116234001 \\
\hline 124 & $36^{\circ} 38^{\prime} 40^{\prime \prime}$ & $116^{\circ} 23^{\prime} 35^{\prime \prime}$ & 155 & 25.4 & -- & 110 & 701.0 & 03-08-55 & 363840116233501 \\
\hline 125 & $36^{\circ} 38^{\prime} 36^{\prime \prime}$ & $116^{\circ} 23^{\prime} 40^{\prime \prime}$ & 163 & 22.86 & -0.5 & 106 & 705.0 & $09-12-90$ & 363836116234001 \\
\hline 126 & $36^{\circ} 38^{\prime} 35^{\prime \prime}$ & $116^{\circ} 23^{\prime} 40^{\prime \prime}$ & 151 & 21.92 & -- & 111 & 700.0 & $04-30-72$ & 363835116234001 \\
\hline 127 & $36^{\circ} 38^{\prime} 30^{\prime \prime}$ & $116^{\circ} 24^{\prime} 14^{\prime \prime}$ & 147 & 25.4 & -0.2 & 98.9 & 712.1 & $01-15-87$ & 363830116241401 \\
\hline 128 & $36^{\circ} 38^{\prime \prime} 25^{\prime \prime}$ & $116^{\circ} 26^{\prime} 32^{\prime \prime}$ & 174 & 35.56 & -0.4 & 90.1 & 705.9 & $01-15-87$ & 363742116263201 \\
\hline 129 & $36^{\circ} 38^{\prime} 15^{\prime \prime}$ & $116^{\circ} 17^{\prime} 59^{\prime \prime}$ & -- & 34.04 & -0.6 & 206 & 725.0 & $09-10-90$ & 363815116175901 \\
\hline 130 & $36^{\circ} 37^{\prime} 49^{\prime \prime}$ & $116^{\circ} 13^{\prime} 02^{\prime \prime}$ & 113 & 15.24 & -. & 102 & 1705.0 & $10-01-59$ & 375017115572201 \\
\hline 131 & $36^{\circ} 37^{\prime} 44^{\prime \prime}$ & $116^{\circ} 26^{\prime} 37^{\prime \prime}$ & 152 & 35.56 & 0.12 & 77.8 & 706.2 & $07-12-62$ & 363711116263701 \\
\hline 132 & $36^{\circ} 37^{\prime} 09^{\prime \prime}$ & $116^{\circ} 26^{\prime} 46^{\prime \prime}$ & -- & 40.64 & -0.4 & 71.7 & 704.3 & $01-15-87$ & 363709116264601 \\
\hline 133 & $36^{\circ} 37^{\prime} 07^{\prime \prime}$ & $116^{\circ} 01^{\prime} 03^{\prime \prime}$ & -. & -- & -- & 297 & 695.0 & $10-10-51$ & 363707116010301 \\
\hline 134 & $36^{\circ} 37^{\prime} 01^{\prime \prime}$ & $116^{\circ} 26^{\prime} 40^{\prime \prime}$ & 135 & 32.25 & -- & 70 & 704.0 & $10-18-58$ & 363621116263201 \\
\hline 135 & $36^{\circ} 36^{\prime} 00^{\prime \prime}$ & $116^{\circ} 01^{\prime} 50^{\prime \prime}$ & 366 & 30.48 & .. & 321 & 640.0 & $03-01-56$ & 363600116015001 \\
\hline 136 & $36^{\circ} 35^{\prime} 49^{\prime \prime}$ & $116^{\circ} 30^{\prime} 50^{\prime \prime}$ & 159 & 35.56 & -0.3 & 46.4 & 695.6 & 01-07-87 & 363549116305001 \\
\hline 137 & $36^{\circ} 35^{\prime} 48^{\prime \prime}$ & $116^{\circ} 35^{\prime} 37^{\prime \prime}$ & 76 & 32.25 & -0.4 & Dry & Dry & $02-13-86$ & 363523116353701 \\
\hline 139 & $36^{\circ} 35^{\prime} 45^{\prime \prime}$ & $116^{\circ} 32^{\prime} 09^{\prime \prime}$ & 101 & 37.33 & -0.2 & 43.2 & 693.8 & $01-08-87$ & 363519116322001 \\
\hline 140 & $36^{\circ} 35^{\prime} 40^{\prime \prime}$ & $116^{\circ} 24^{\prime} 08^{\prime \prime}$ & 95 & 32.25 & -. & 48.9 & 722.1 & 03-13-73 & 363540116240801 \\
\hline 141 & $36^{\circ} 35^{\prime} 34^{\prime \prime}$ & $116^{\circ} 01^{\prime} 55^{\prime \prime}$ & 593 & 34.80 & -- & 239 & 722.0 & $10-15-87$ & 363517116021501 \\
\hline 142 & $36^{\circ} 35^{\prime} 30^{\prime \prime}$ & $116^{\circ} 02^{\prime} 14^{\prime \prime}$ & -- & 45.72 & .- & 239 & 722.0 & $11-07-63$ & 363530116021401 \\
\hline 143 & $36^{\circ} 35^{\prime} 27^{\prime \prime}$ & $116^{\circ} 29^{\prime} 25^{\prime \prime}$ & 107 & 35.56 & -0.2 & 47.1 & 696.9 & $01-16-87$ & 363527116292501 \\
\hline 144 & $36^{\circ} 35^{\prime} 21^{\prime \prime}$ & $116^{\circ} 35^{\prime} 25^{\prime \prime}$ & 78 & 40.64 & -0.4 & 39.3 & 690.7 & 01-07-87 & 363521116352501 \\
\hline 145 & $36^{\circ} 35^{\prime} 15^{\prime \prime}$ & $116^{\circ} 33^{\prime} 55^{\prime \prime}$ & 94 & 32.25 & 0.12 & 33.0 & 708.0 & $07-02-62$ & 363456116335501 \\
\hline
\end{tabular}


Table 1. Ground-water levels and well data of wells located in the Beatty, Nevada-California, 30-minute $\times 60$-minute quadrangle--Continued

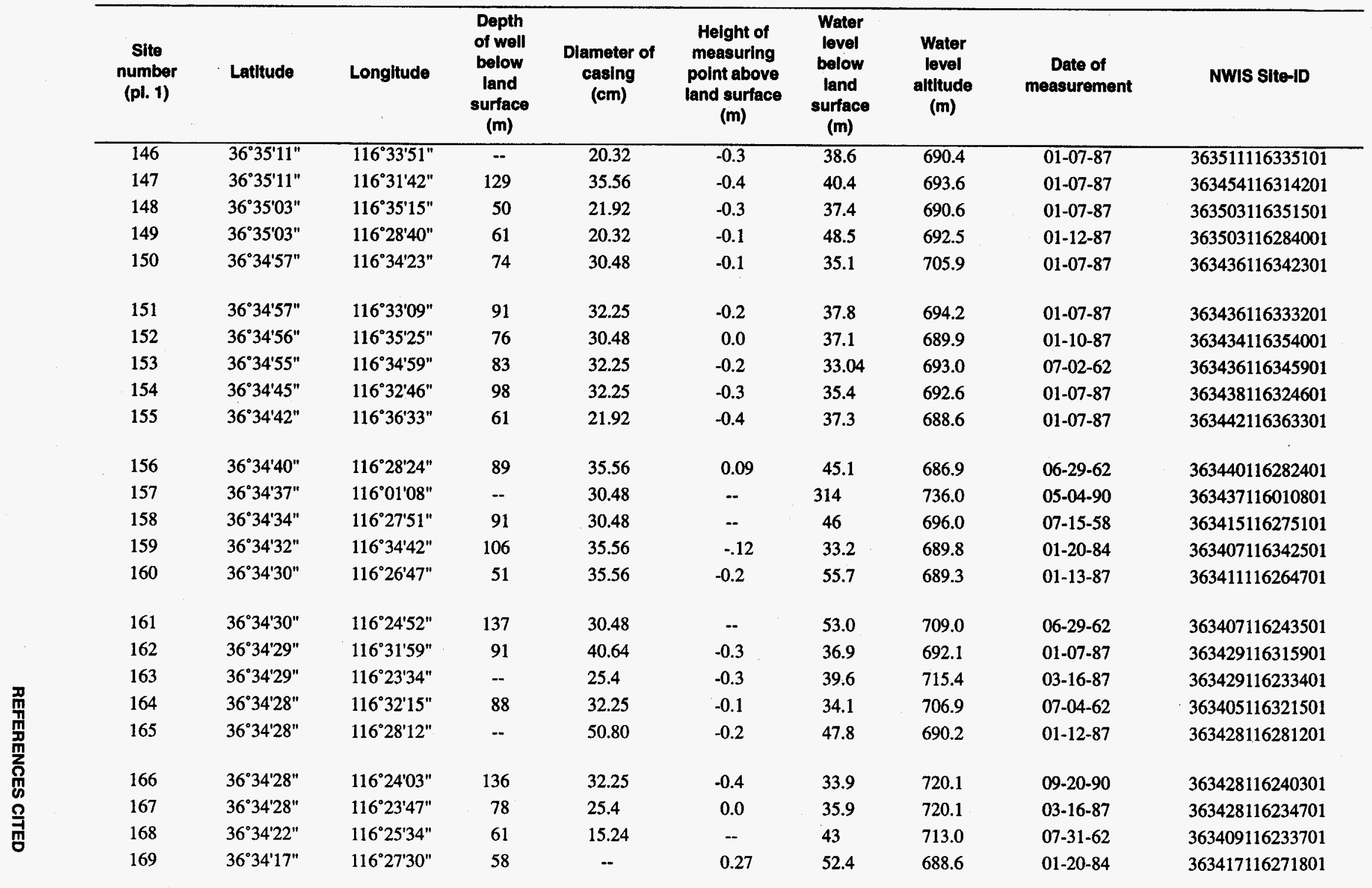




\begin{tabular}{|c|c|c|c|c|c|c|c|c|c|}
\hline $\begin{array}{c}\text { Site } \\
\text { number } \\
\text { (pl. 1) }\end{array}$ & Latitude & Longitude & $\begin{array}{l}\text { Depth } \\
\text { of well } \\
\text { below } \\
\text { land } \\
\text { surface } \\
\text { (m) }\end{array}$ & $\begin{array}{l}\text { Diameter of } \\
\text { casing } \\
\text { (cm) }\end{array}$ & $\begin{array}{c}\text { Height of } \\
\text { measuring } \\
\text { point above } \\
\text { land surface } \\
\text { (m) }\end{array}$ & $\begin{array}{l}\text { Water } \\
\text { level } \\
\text { below } \\
\text { land } \\
\text { surface } \\
\text { (m) }\end{array}$ & $\begin{array}{l}\text { Water } \\
\text { level } \\
\text { altitude } \\
\text { (m) }\end{array}$ & $\begin{array}{c}\text { Date of } \\
\text { measurement }\end{array}$ & NWIS Site-ID \\
\hline 170 & $36^{\circ} 34^{\prime} 10^{\prime \prime}$ & $116^{\circ} 26^{\prime} 11^{\prime \prime}$ & 204 & 35.56 & -0.3 & 52.5 & 691.5 & $01-15-87$ & 363410116261101 \\
\hline 171 & $36^{\circ} 34^{\prime} 10^{\prime \prime}$ & $116^{\circ} 24^{\prime} 03^{\prime \prime}$ & 61 & 16.82 & -0.2 & 34.6 & 714.4 & 03-16-87 & 363410116240301 \\
\hline 172 & $36^{\circ} 34^{\prime} 10^{\prime \prime}$ & $116^{\circ} 24^{\prime} 00^{\prime \prime}$ & 54 & 32.25 & -0.2 & 26.2 & 723.8 & $03-16-87$ & 363410116240001 \\
\hline 173 & $36^{\circ} 34^{\prime} 05^{\prime \prime}$ & $116^{\circ} 33^{\prime} 45^{\prime \prime}$ & 76 & 30.48 & -- & 27 & 697.0 & $08-15-58$ & 363342116335701 \\
\hline 174 & $36^{\circ} 34^{\prime} 04^{\prime \prime}$ & $116^{\circ} 33^{\prime} 12^{\prime \prime}$ & 92 & 35.56 & -0.2 & 31.8 & 692.2 & $01-08-87$ & 363340116332901 \\
\hline 175 & $36^{\circ} 34^{\prime} 04^{\prime \prime}$ & $116^{\circ} 32^{\prime} 39^{\prime \prime}$ & 46 & 35.56 & -0.3 & 31.5 & 692.5 & $02-26-74$ & 363342116325101 \\
\hline 176 & $36^{\circ} 34^{\prime} 04^{\prime \prime}$ & $116^{\circ} 25^{\prime} 04^{\prime \prime}$ & 88 & 30.48 & -0.1 & 48.2 & 698.8 & $06-29-62$ & 363350116252101 \\
\hline 177 & $36^{\circ} 34^{\prime} 02^{\prime \prime}$ & $116^{\circ} 34^{\prime} 42^{\prime \prime}$ & -- & 21.92 & -0.3 & 31.9 & 689.1 & $01-07-87$ & 363402116344201 \\
\hline 178 & $36^{\circ} 34^{\prime} 01^{\prime \prime}$ & $116^{\circ} 35^{\prime} 10^{\prime \prime}$ & 85 & 30.48 & -0.1 & 33.2 & 688.8 & $01-20-84$ & 363342116345401 \\
\hline 179 & $36^{\circ} 34^{\prime} 01^{\prime \prime}$ & $116^{\circ} 26^{\prime} 17^{\prime \prime}$ & 119 & 32.25 & 0.0 & 49.8 & 691.2 & $01-20-84$ & 363348116261701 \\
\hline 180 & $36^{\circ} 34^{\prime} 00^{\prime \prime}$ & $116^{\circ} 32^{\prime} 06^{\prime \prime}$ & 90 & 35.56 & -0.3 & 34.0 & 692.0 & 01-08-87 & 363346116322801 \\
\hline 181 & $36^{\circ} 33^{\prime} 56^{\prime \prime}$ & $116^{\circ} 29^{\prime} 45^{\prime \prime}$ & 128 & 32.25 & -0.1 & 35.4 & 691.6 & $06-28-62$ & 363348116292701 \\
\hline 182 & $36^{\circ} 33^{\prime} 55^{\prime \prime}$ & $116^{\circ} 25^{\prime} 49^{\prime \prime}$ & 139 & 32.25 & -0.2 & 50.0 & 692.0 & $01-15-87$ & 363355116254901 \\
\hline 183 & $36^{\circ} 33^{\prime} 53^{\prime \prime}$ & $116^{\circ} 30^{\prime} 33 "$ & 76 & 35.56 & -0.5 & 37.2 & 689.8 & $01-08-87$ & 363344116304501 \\
\hline 184 & $36^{\circ} 33^{\prime} 52^{\prime \prime}$ & $116^{\circ} 36^{\prime} 28^{\prime \prime}$ & 110 & 40.64 & -0.2 & 35.8 & 684.2 & $01-20-84$ & 363340116362801 \\
\hline 185 & $36^{\circ} 33^{\prime} 48^{\prime \prime}$ & $116^{\circ} 25^{\prime} 49^{\prime \prime}$ & 119 & 32.25 & -0.1 & 51.1 & 692.9 & $03-21-86$ & 363348116254901 \\
\hline 186 & $36^{\circ} 33^{\prime} 38^{\prime \prime}$ & $116^{\circ} 30^{\prime} 31^{\prime \prime}$ & 91 & 30.48 & -- & Dry & Dry & $01-08-87$ & 363338116303101 \\
\hline 187 & $36^{\circ} 33^{\prime} 36^{\prime \prime}$ & $116^{\circ} 29^{\prime} 30^{\prime \prime}$ & 76 & -- & -0.2 & 35.3 & 690.7 & $06-28-62$ & 363317116293001 \\
\hline 188 & $36^{\circ} 33^{\prime} 33^{\prime \prime}$ & $116^{\circ} 06^{\prime} 42^{\prime \prime}$ & -- & -- & -- & 138.5 & 720.5 & $04-29-64$ & 363333116064201 \\
\hline 189 & $36^{\circ} 33^{\prime} 32^{\prime \prime}$ & $116^{\circ} 32^{\prime} 50^{\prime \prime}$ & 61 & 21.92 & 0.0 & 29.4 & 691.6 & $01-08-87$ & 363332116323501 \\
\hline 190 & $36^{\circ} 33^{\prime} 16^{\prime \prime}$ & $116^{\circ} 36^{\prime} 24^{\prime \prime}$ & 53 & 21.92 & -0.3 & 30.1 & 688.9 & $01-07-87$ & 363316116362401 \\
\hline 191 & $36^{\circ} 33^{\prime} 15^{\prime \prime}$ & $116^{\circ} 30^{\prime} 31^{\prime \prime}$ & -- & -- & -0.1 & 35.1 & 686.9 & $01-19-84$ & 363248116303101 \\
\hline 192 & $36^{\circ} 33^{\prime} 11^{\prime \prime}$ & $116^{\circ} 31^{\prime} 39^{\prime \prime}$ & 101 & 35.56 & -0.2 & 33.1 & 685.9 & $01-10-87$ & 363254116313901 \\
\hline 193 & $36^{\circ} 33^{\prime} 10^{\prime \prime}$ & $116^{\circ} 29^{\prime} 40^{\prime \prime}$ & 106 & 30.48 & 0.0 & 36.1 & 687.9 & 03-28-91 & 363310116294001 \\
\hline
\end{tabular}


Table 1. Ground-water levels and well data of wells located in the Beatty, Nevada-Califomia, 30-minute $\times 60$-minute quadrangle--Continued

\begin{tabular}{|c|c|c|c|c|c|c|c|c|c|}
\hline $\begin{array}{l}\text { Site } \\
\text { number } \\
\text { (pl. 1) }\end{array}$ & Latitude & Longitude & $\begin{array}{l}\text { Depth } \\
\text { of well. } \\
\text { below } \\
\text { land } \\
\text { surface } \\
\text { (m) }\end{array}$ & $\begin{array}{l}\text { Diameter of } \\
\text { casing } \\
\text { (cm) }\end{array}$ & $\begin{array}{l}\text { Height of } \\
\text { measuring } \\
\text { point above } \\
\text { land surface } \\
\text { (m) }\end{array}$ & $\begin{array}{l}\text { Water } \\
\text { level } \\
\text { below } \\
\text { land } \\
\text { surface } \\
(m)\end{array}$ & $\begin{array}{l}\text { Water } \\
\text { level } \\
\text { altitude } \\
\text { (m) }\end{array}$ & $\begin{array}{c}\text { Date of } \\
\text { measurement }\end{array}$ & NWIS Site-ID \\
\hline 194 & $36^{\circ} 33^{\prime} 09^{\prime \prime}$ & $116^{\circ} 28^{\prime} 21^{\prime \prime}$ & 91 & 35.56 & -0.2 & 39.2 & 687.8 & $01-13-87$ & 363256116282101 \\
\hline 195 & $36^{\circ} 33^{\prime} 09^{\prime \prime}$ & $116^{\circ} 26^{\prime} 50^{\prime \prime}$ & 107 & 30.48 & -0.4 & 42.8 & 687.2 & $01-13-87$ & 363252116265001 \\
\hline 196 & $36^{\circ} 33^{\prime} 03^{\prime \prime}$ & $116^{\circ} 30^{\prime} 01^{\prime \prime}$ & 221 & 35.56 & -0.3 & 34.8 & 688.2 & $01-10-87$ & 363254116295501 \\
\hline 197 & $36^{\circ} 33^{\prime} 03^{\prime \prime}$ & $116^{\circ} 29^{\prime} 29^{\prime \prime}$ & 146 & 35.56 & -- & Dry & Dry & $12-11-63$ & 363252116292501 \\
\hline 198 & $36^{\circ} 33^{\prime} 03^{\prime \prime}$ & $116^{\circ} 25^{\prime} 13^{\prime \prime}$ & 152 & 30.48 & -0.6 & 32.8 & 699.2 & $01-13-87$ & 363250116251301 \\
\hline 199 & $36^{\circ} 33^{\prime} 02^{\prime \prime}$ & $116^{\circ} 32^{\prime} 18^{\prime \prime}$ & 101 & 30.48 & -. & 29 & 690.0 & $03-23-60$ & 363244116320701 \\
\hline 200 & $36^{\circ} 32^{\prime} 48^{\prime \prime}$ & $116^{\circ} 30^{\prime} 28^{\prime \prime}$ & -- & 16.84 & -0.1 & 32.6 & 685.4 & 01-10-87 & 363248116302801 \\
\hline 201 & $36^{\circ} 32^{\prime} 47^{\prime \prime}$ & $116^{\circ} 31^{\prime} 29^{\prime \prime}$ & 91 & 35.56 & -0.3 & 32.2 & 683.8 & $01-10-87$ & 363247116312901 \\
\hline 202 & $36^{\circ} 32^{\prime} 46^{\prime \prime}$ & $116^{\circ} 29^{\prime} 22^{\prime \prime}$ & 94 & 35.56 & 0.15 & 30.0 & 690.0 & $07-09-62$ & 363223116292201 \\
\hline 203 & $36^{\circ} 32^{\prime} 43^{\prime \prime}$ & $116^{\circ} 35^{\prime} 41^{\prime \prime}$ & -- & -- & -0.5 & 26.7 & 687.3 & $01-08-87$ & 363243116354101 \\
\hline 204 & $36^{\circ} 32^{\prime} 42^{\prime \prime}$ & $116^{\circ} 30^{\prime} 19^{\prime \prime}$ & 91 & 32.25 & 0.0 & 32.1 & 686.9 & $01-10-87$ & 363223116295501 \\
\hline 205 & $36^{\circ} 32^{\prime} 42^{\prime \prime}$ & $116^{\circ} 28^{\prime} 39^{\prime \prime}$ & 125 & 30.48 & -- & 34 & 687.0 & 03-10-59 & 363225116282401 \\
\hline 206 & $36^{\circ} 32^{\prime} 39^{\prime \prime}$ & $116^{\circ} 31^{\prime} 44^{\prime \prime}$ & 153 & 35.56 & -0.4 & 23.7 & 723.3 & $07-07-62$ & 363221116313901 \\
\hline 207 & $36^{\circ} 32^{\prime} 39^{\prime \prime}$ & $116^{\circ} 31^{\prime} 09^{\prime \prime}$ & 88 & 32.25 & -0.1 & 25.6 & 689.4 & $07-09-62$ & 363225116310901 \\
\hline 208 & $36^{\circ} 32^{\prime} 37^{\prime \prime}$ & $116^{\circ} 30^{\prime} 33^{\prime \prime}$ & 128 & 35.56 & 0.0 & 28.2 & 689.8 & $12-02-60$ & 363223116303801 \\
\hline 209 & $36^{\circ} 32^{\prime} 37^{\prime \prime}$ & $116^{\circ} 29^{\prime} 29^{\prime \prime}$ & 91 & 35.56 & 0.0 & 32.8 & 687.2 & $01-10-87$ & 363237116292901 \\
\hline 210 & $36^{\circ} 32^{\prime} 36^{\prime \prime}$ & $116^{\circ} 26^{\prime} 31^{\prime \prime}$ & 112 & 40.64 & 0.0 & 36.9 & 685.1 & $02-12-86$ & 363225116260901 \\
\hline 211 & $36^{\circ} 32^{\prime} 34^{\prime \prime}$ & $116^{\circ} 32^{\prime} 17^{\prime \prime}$ & 148 & 35.56 & -0.2 & 22.9 & 691.1 & $07-07-62$ & 363217116320001 \\
\hline 212 & $36^{\circ} 32^{\prime} 30^{\prime \prime}$ & $116^{\circ} 26^{\prime} 05^{\prime \prime}$ & -- & 20.32 & -0.4 & 36.8 & 686.2 & $01-13-87$ & 363230116260501 \\
\hline 213 & $36^{\circ} 32^{\prime} 23^{\prime \prime}$ & $116^{\circ} 34^{\prime} 59^{\prime \prime}$ & 80 & 40.64 & -1.0 & 19.0 & 694.0 & $07-04-62$ & 363223116345901 \\
\hline 214 & $36^{\circ} 32^{\prime} 19^{\prime \prime}$ & $116^{\circ} 29^{\prime} 58^{\prime \prime}$ & 90 & 35.56 & 0.1 & Dry & Dry & $01-14-87$ & 363203116295801 \\
\hline 215 & $36^{\circ} 32^{\prime} 18^{\prime \prime}$ & $116^{\circ} 31^{\prime} 34^{\prime \prime}$ & 42 & -- & $-\cdot$ & 30.6 & 681.4 & $01-19-84$ & 363200116313401 \\
\hline 216 & $36^{\circ} 32^{\prime} 17^{\prime \prime}$ & $116^{\circ} 27^{\prime} 18^{\prime \prime}$ & 91 & 40.64 & -0.2 & 35.4 & 686.6 & $01-13-87$ & 363205116271801 \\
\hline 217 & $36^{\circ} 32^{\prime} 17^{\prime \prime}$ & $116^{\circ} 26^{\prime} 58^{\prime \prime}$ & 76 & 40.64 & -0.3 & 36.1 & 685.9 & $01-13-87$ & 363212116270401 \\
\hline
\end{tabular}




\begin{tabular}{|c|c|c|c|c|c|c|c|c|c|}
\hline $\begin{array}{c}\text { Site } \\
\text { number } \\
\text { (pl. 1) }\end{array}$ & Latitude & Longitude & $\begin{array}{l}\text { Depth } \\
\text { of well } \\
\text { below } \\
\text { land } \\
\text { surface } \\
\text { (m) }\end{array}$ & $\begin{array}{l}\text { Diameter of } \\
\text { casing } \\
\text { (cm) }\end{array}$ & $\begin{array}{c}\text { Height of } \\
\text { measuring } \\
\text { point above } \\
\text { land surface } \\
\text { (m) }\end{array}$ & $\begin{array}{l}\text { Water } \\
\text { level } \\
\text { below } \\
\text { land } \\
\text { surface } \\
\text { (m) }\end{array}$ & $\begin{array}{l}\text { Water } \\
\text { level } \\
\text { altitude } \\
(\mathrm{m})\end{array}$ & $\begin{array}{c}\text { Date of } \\
\text { measurement }\end{array}$ & NWIS Sito-ID \\
\hline 218 & $36^{\circ} 32^{\prime} 14^{\prime \prime}$ & $116^{\circ} 28^{\prime} 48^{\prime \prime}$ & 61 & 16.84 & -0.2 & 31.7 & 686.3 & $01-14-87$ & 363214116284801 \\
\hline 219 & $36^{\circ} 32^{\prime} 14^{\prime \prime}$ & $116^{\circ} 13^{\prime} 35^{\prime \prime}$ & 312 & 51.31 & - & 13.5 & 719.5 & $10-18-66$ & 363214116133501 \\
\hline 220 & $36^{\circ} 32^{\prime} 13^{\prime \prime}$ & $116^{\circ} 13^{\prime} 40^{\prime \prime}$ & 189 & 11.43 & -0.4 & 13.4 & 719.6 & $03-15-87$ & 363213116134001 \\
\hline 221 & $36^{\circ} 32^{\prime} 13^{\prime \prime}$ & $116^{\circ} 13^{\prime} 39^{\prime \prime}$ & 249 & 35.56 & -0.4 & 12.1 & 719.9 & $03-15-87$ & 363213116133901 \\
\hline 222 & $36^{\circ} 32^{\prime} 13^{\prime \prime}$ & $116^{\circ} 13^{\prime} 38^{\prime \prime}$ & 203 & 19.38 & -0.1 & 12.7 & 719.3 & $03-15-87$ & 363213116133800 \\
\hline 223 & $36^{\circ} 32^{\prime} 13^{\prime \prime}$ & $116^{\circ} 13 ' 37^{\prime \prime}$ & 202 & 19.38 & -0.3 & 13.5 & 719.5 & $03-15-87$ & 363213116133700 \\
\hline 224 & $36^{\circ} 32^{\prime} 11^{\prime \prime}$ & $116^{\circ} 30^{\prime} 32^{\prime \prime}$ & 50 & 40.64 & -0.1 & 30.9 & 683.1 & $01-08-87$ & 363200116304401 \\
\hline 225 & $36^{\circ} 32^{\prime} 11^{\prime \prime}$ & $116^{\circ} 29^{\prime} 46^{\prime \prime}$ & 55 & 40.64 & -- & Dry & Dry & $12-11-63$ & 363211116294601 \\
\hline 226 & $36^{\circ} 32^{\prime} 10^{\prime \prime}$ & $116^{\circ} 31^{\prime} 38^{\prime \prime}$ & 61 & 40.64 & 0.0 & 30.0 & 683.0 & $01-13-87$ & 363210116313801 \\
\hline 227 & $36^{\circ} 32^{\prime} 09^{\prime \prime}$ & $116^{\circ} 31^{\prime} 40^{\prime \prime}$ & 60 & -- & -- & 23.3 & 677.7 & $02-24-75$ & 363209116314001 \\
\hline 228 & $36^{\circ} 32^{\prime} 00^{\prime \prime}$ & $116^{\circ} 32^{\prime} 33^{\prime \prime}$ & 61 & 30.48 & -- & 16 & 694.0 & $03-12-56$ & 363200116323301 \\
\hline 229 & $36^{\circ} 31^{\prime} 57^{\prime \prime}$ & $116^{\circ} 22^{\prime} 12^{\prime \prime}$ & -- & 20.32 & -0.3 & 30.6 & 694.4 & $03-15-87$ & 363157116221201 \\
\hline 230 & $36^{\circ} 31^{\prime} 55^{\prime \prime}$ & $116^{\circ} 32^{\prime} 33^{\prime \prime}$ & 72 & 30.48 & -- & 18 & 690.0 & $01-27-66$ & 363155116323301 \\
\hline 231 & $36^{\circ} 31^{\prime} 50^{\prime \prime}$ & $116^{\circ} 31^{\prime} 16^{\prime \prime}$ & 16 & 35.56 & 0.0 & 20.4 & 688.6 & $07-09-62$ & 363138116310101 \\
\hline 232 & $36^{\circ} 31^{\prime} 48^{\prime \prime}$ & $116^{\circ} 32^{\prime} 38^{\prime \prime}$ & 57 & 30.48 & -0.2 & 22.5 & 685.5 & $01-10-87$ & 363138116323801 \\
\hline 233 & $36^{\circ} 31^{\prime} 48^{\prime \prime}$ & $116^{\circ} 25^{\prime} 10^{\prime \prime}$ & 59 & 20.32 & -0.4 & 40.7 & 675.3 & $01-15-87$ & 363148116251001 \\
\hline 234 & $36^{\circ} 31^{\prime} 46^{\prime \prime}$ & $116^{\circ} 27^{\prime} 20^{\prime \prime}$ & 91 & 40.64 & 0.03 & 29.5 & 687.5 & $06-26-62$ & 363136116271501 \\
\hline 235 & $36^{\circ} 31^{\prime} 46^{\prime \prime}$ & $116^{\circ} 25^{\prime} 27^{\prime \prime}$ & 91 & 35.56 & 0.2 & 25.0 & 692.0 & $06-26-62$ & 363138116252101 \\
\hline 236 & $36^{\circ} 31^{\prime} 45^{\prime \prime}$ & $116^{\circ} 31^{\prime} 06^{\prime \prime}$ & 61 & 40.64 & -0.1 & Dry & Dry & 08-16-78 & 363145116310601 \\
\hline 237 & $36^{\circ} 31^{\prime} 38^{\prime \prime}$ & $116^{\circ} 25^{\prime} 05^{\prime \prime}$ & 91 & 27.17 & - & 32.5 & -- & $06-26-62$ & 363138116250501 \\
\hline 238 & $36^{\circ} 31^{\prime} 38^{\prime \prime}$ & $116^{\circ} 25^{\prime} 05^{\prime \prime}$ & 61 & 20.32 & -- & 38 & -- & $06-25-61$ & 363138116250502 \\
\hline 240 & $36^{\circ} 31^{\prime} 34^{\prime \prime}$ & $116^{\circ} 29^{\prime} 20^{\prime \prime}$ & 55 & 21.89 & -0.3 & 25.5 & 684.5 & $01-14-87$ & 363134116292001 \\
\hline 241 & $36^{\circ} 31^{\prime} 33^{\prime \prime}$ & $116^{\circ} 25^{\prime} 01^{\prime \prime}$ & 49 & 20.32 & -0.2 & 37.9 & 675.1 & $01-12-87$ & 363133116250101 \\
\hline 242 & $36^{\circ} 31^{\prime} 32^{\prime \prime}$ & $116^{\circ} 28 ' 57^{\prime \prime}$ & 25 & -- & -- & 24.7 & 688.3 & $06-26-62$ & 363138116290201 \\
\hline
\end{tabular}


Table 1. Ground-water levels and well data of wells located in the Beatty, Nevada-California, 30-minute $\times 60$-minute quadrangle--Continued

\begin{tabular}{|c|c|c|c|c|c|c|c|c|c|}
\hline $\begin{array}{c}\text { Site } \\
\text { number } \\
\text { (pl. 1) }\end{array}$ & Latitude & Longitude & $\begin{array}{l}\text { Depth } \\
\text { of well } \\
\text { below } \\
\text { land } \\
\text { surface } \\
(\mathrm{m})\end{array}$ & $\begin{array}{l}\text { Diameter of } \\
\text { casing } \\
\text { (cm) }\end{array}$ & $\begin{array}{l}\text { Height of } \\
\text { measuring } \\
\text { point above } \\
\text { land surface } \\
\text { (m) }\end{array}$ & $\begin{array}{l}\text { Water } \\
\text { lovel } \\
\text { below } \\
\text { land } \\
\text { surface } \\
\text { (m) }\end{array}$ & $\begin{array}{l}\text { Water } \\
\text { level } \\
\text { altitude } \\
\text { (m) }\end{array}$ & $\begin{array}{c}\text { Date of } \\
\text { measurement }\end{array}$ & NWIS Site-ID \\
\hline 243 & $36^{\circ} 31^{\prime} 32^{\prime \prime}$ & $116^{\circ} 28^{\prime} 52^{\prime \prime}$ & - & 20.32 & -0.3 & 25.5 & 685.5 & $01-14-87$ & 363132116285201 \\
\hline 244 & $36^{\circ} 31^{\prime} 32^{\prime \prime}$ & $116^{\circ} 27^{\prime} 53^{\prime \prime}$ & 61 & 40.64 & -0.2 & 33.8 & 682.2 & $01-18-84$ & 363140116275301 \\
\hline 245 & $36^{\circ} 31^{\prime} 29^{\prime \prime}$ & $116^{\circ} 25^{\prime} 29^{\prime \prime}$ & 61 & 21.92 & -0.2 & 36.8 & 676.2 & $01-12-87$ & 363129116252901 \\
\hline 248 & $36^{\circ} 31^{\prime} 28^{\prime \prime}$ & $116^{\circ} 25^{\prime} 31^{\prime \prime}$ & 68 & 35.56 & $-\cdot$ & 26 & 688.0 & $03-15-59$ & 363109116253101 \\
\hline 249 & $36^{\circ} 31^{\prime} 23^{\prime \prime}$ & $116^{\circ} 30^{\prime} 00^{\prime \prime}$ & 47 & -. & -- & 21.1 & 687.9 & $06-26-62$ & 363111116300001 \\
\hline 250 & $36^{\circ} 31^{\prime} 21^{\prime \prime}$ & $116^{\circ} 11^{\prime} 23^{\prime \prime}$ & 46 & 20.32 & -- & 30.3 & 714.7 & $08-30-62$ & 363121116112301 \\
\hline 251 & $36^{\circ} 31^{\prime} 20^{\prime \prime}$ & $116^{\circ} 30^{\prime} 00^{\prime \prime}$ & 49 & 40.64 & 0.0 & Dry & Dry & $03-28-91$ & 363120116300001 \\
\hline 252 & $36^{\circ} 31^{\prime} 17^{\prime \prime}$ & $116^{\circ} 30^{\prime} 36^{\prime \prime}$ & 49 & 35.56 & -0.1 & 25.3 & 683.7 & $01-10-87$ & 363117116303601 \\
\hline 257 & $36^{\circ} 31^{\prime} 00^{\prime \prime}$ & $116^{\circ} 28^{\prime} 14^{\prime \prime}$ & 77 & 32.25 & -0.4 & 24.7 & 682.3 & $01-14-87$ & 363046116281401 \\
\hline 258 & $36^{\circ} 30^{\prime} 58^{\prime \prime}$ & $116^{\circ} 31^{\prime} 28^{\prime \prime}$ & 91 & 40.64 & -0.2 & 19.9 & 684.1 & $01-10-87$ & 363058116312801 \\
\hline 259 & $36^{\circ} 30^{\prime} 58^{\prime \prime}$ & $116^{\circ} 28^{\prime} 57^{\prime \prime}$ & 24 & -- & -- & 19.4 & 686.6 & $06-26-62$ & 363046116285701 \\
\hline 260 & $36^{\circ} 30^{\prime} 58^{\prime \prime}$ & $116^{\circ} 27^{\prime} 05^{\prime \prime}$ & 113 & 35.56 & -0.2 & 29.3 & 677.7 & $01-16-87$ & 363058116270501 \\
\hline 261 & $36^{\circ} 30^{\prime} 54^{\prime \prime}$ & $116^{\circ} 27^{\prime} 04^{\prime \prime}$ & 91 & 35.56 & - & 28 & 679.0 & $07-06-62$ & 363054116270401 \\
\hline 262 & $36^{\circ} 30^{\prime} 53^{\prime \prime}$ & $116^{\circ} 27^{\prime} 20^{\prime \prime}$ & 92 & 35.56 & -- & 25.0 & 682.0 & $04-16-68$ & 363053116272001 \\
\hline 263 & $36^{\circ} 30^{\prime} 53^{\prime \prime}$ & $116^{\circ} 27^{\prime} 10^{\prime \prime}$ & -- & 30.48 & -0.4 & 27.7 & 677.3 & $01-14-87$ & 363053116271001 \\
\hline 264 & $36^{\circ} 30^{\prime} 50^{\prime \prime}$ & $116^{\circ} 10^{\prime} 50^{\prime \prime}$ & 41 & 20.95 & - & 18 & 714.0 & $02-01-61$ & 363050116105001 \\
\hline 265 & $36^{\circ} 30^{\prime} 44^{\prime \prime}$ & $116^{\circ} 49^{\prime} 15^{\prime \prime}$ & 6.0 & -- & -0.18 & .62 & 289.4 & $01-23-86$ & 363044116491502 \\
\hline 266 & $36^{\circ} 30^{\prime} 44^{\prime \prime}$ & $116^{\circ} 30^{\prime} 50^{\prime \prime}$ & 124 & 35.56 & -- & 16.6 & 685.4 & $01-14-69$ & 363044116303601 \\
\hline
\end{tabular}




\begin{tabular}{|c|c|c|c|c|c|c|c|c|c|}
\hline $\begin{array}{l}\text { Site } \\
\text { number } \\
\text { (pl. 1) }\end{array}$ & Latitude & Longitude & $\begin{array}{l}\text { Depth } \\
\text { of well } \\
\text { below } \\
\text { land } \\
\text { surface } \\
\text { (m) }\end{array}$ & $\begin{array}{l}\text { Diameter of } \\
\text { casing } \\
\text { (cm) }\end{array}$ & $\begin{array}{l}\text { Height of } \\
\text { measuring } \\
\text { point above } \\
\text { land surface } \\
(\mathrm{m})\end{array}$ & $\begin{array}{l}\text { Water } \\
\text { level } \\
\text { below } \\
\text { land } \\
\text { surface } \\
\text { (m) }\end{array}$ & $\begin{array}{l}\text { Water } \\
\text { level } \\
\text { altitude } \\
(\mathrm{m})\end{array}$ & $\begin{array}{c}\text { Date of } \\
\text { measurement }\end{array}$ & NWIS Site-ID \\
\hline 267 & $36^{\circ} 30^{\prime} 44^{\prime \prime}$ & $116^{\circ} 27^{\prime} 32^{\prime \prime}$ & 98 & -- & 0.2 & 25.5 & 715.5 & $06-20-62$ & 363044116271501 \\
\hline 268 & $36^{\circ} 30^{\prime} 39^{\prime \prime}$ & $116^{\circ} 30^{\prime} 35^{\prime \prime}$ & $\ldots$ & 30.48 & -0.2 & 21.1 & 681.9 & $01-12-87$ & 363039116303501 \\
\hline 269 & $36^{\circ} 30^{\prime} 30^{\prime \prime}$ & $116^{\circ} 28^{\prime} 00^{\prime \prime}$ & -- & -- & -- & 21 & 685.0 & $01-20-56$ & 363030116280001 \\
\hline 270 & $36^{\circ} 30^{\prime} 30^{\prime \prime}$ & $116^{\circ} 10^{\prime} 45^{\prime \prime}$ & 31 & 20.32 & -0.3 & 18.7 & 713.3 & $01-16-87$ & 363030116104501 \\
\hline 271 & $36^{\circ} 30^{\prime} 29^{\prime \prime}$ & $116^{\circ} 29^{\prime} 24^{\prime \prime}$ & 21 & -- & - & 18 & 686.0 & $12-22-55$ & 363009116291101 \\
\hline 272 & $36^{\circ} 30^{\prime} 29^{\prime \prime}$ & $116^{\circ} 28^{\prime} 04^{\prime \prime}$ & 28 & -- & -- & 25.7 & 680.3 & $06-26-62$ & 363011116280401 \\
\hline 273 & $36^{\circ} 30^{\prime} 28^{\prime \prime}$ & $116^{\circ} 27^{\prime} 02^{\prime \prime}$ & 107 & 35.56 & -- & 26.8 & 675.2 & $03-21-86$ & 363028116270201 \\
\hline 274 & $36^{\circ} 30^{\prime} 28^{\prime \prime}$ & $116^{\circ} 25^{\prime} 30^{\prime \prime}$ & 91 & 40.64 & 0.0 & 24.8 & 676.2 & $06-26-62$ & 363017116253001 \\
\hline 275 & $36^{\circ} 30^{\prime} 27^{\prime \prime}$ & $116^{\circ} 30^{\prime} 27^{\prime \prime}$ & 41 & 40.64 & -- & 20.9 & 681.1 & $12-10-85$ & 363009116302701 \\
\hline 276 & $36^{\circ} 30^{\prime} 27^{\prime \prime}$ & $116^{\circ} 29^{\prime} 52^{\prime \prime}$ & 47 & 32.25 & -0.4 & 20.1 & 682.9 & $01-12-87$ & 363027116295201 \\
\hline 277 & $36^{\circ} 30^{\prime} 27^{\prime \prime}$ & $116^{\circ} 27^{\prime} 00^{\prime \prime}$ & 180 & 35.56 & -- & 25 & 677.0 & $10-17-62$ & 363027116270001 \\
\hline 278 & $36^{\circ} 30^{\prime} 26^{\prime \prime}$ & $116^{\circ} 27^{\prime} 56^{\prime \prime}$ & 80 & 35.56 & -0.3 & 28.2 & 677.8 & $01-12-87$ & 363026116275601 \\
\hline 279 & $36^{\circ} 30^{\prime} 26^{\prime \prime}$ & $116^{\circ} 16^{\prime} 04^{\prime \prime}$ & -- & -- & -- & 24.5 & 685.5 & 03-09-72 & 363026116160401 \\
\hline 280 & $36^{\circ} 30^{\prime} 26^{\prime \prime}$ & $116^{\circ} 16^{\prime} 04^{\prime \prime}$ & -- & -. & -- & 30.7 & 679.3 & $03-09-72$ & 363026116160402 \\
\hline 281 & $36^{\circ} 30^{\prime} 20^{\prime \prime}$ & $116^{\circ} 29^{\prime} 00^{\prime \prime}$ & 24 & -- & -. & 20 & 681.0 & $01-17-56$ & 363020116290001 \\
\hline 282 & $36^{\circ} 30^{\prime} 17^{\prime \prime}$ & $116^{\circ} 25^{\prime} 30^{\prime \prime}$ & 61 & 30.48 & - & 18 & 685.0 & $05-07-57$ & 363017116253002 \\
\hline 283 & $36^{\circ} 30^{\prime} 05^{\prime \prime}$ & $116^{\circ} 29^{\prime} 10^{\prime \prime}$ & -- & -- & -- & 17 & 683.0 & $01-10-56$ & 363005116291001 \\
\hline 284 & $36^{\circ} 30^{\prime} 02^{\prime \prime}$ & $116^{\circ} 31^{\prime} 07^{\prime \prime}$ & 65 & 35.56 & -- & 13.6 & 681.4 & 03-13-73 & 363002116310701 \\
\hline 285 & $36^{\circ} 30^{\prime} 2^{\prime \prime}$ & $116^{\circ} 30^{\prime} 48^{\prime \prime}$ & -- & 35.56 & -0.2 & 17.4 & 680.6 & $01-12-87$ & 363002116304801 \\
\hline 286 & $36^{\circ} 30^{\prime} 01^{\prime \prime}$ & $116^{\circ} 25^{\prime} 41^{\prime \prime}$ & 58 & 30.48 & -0.3 & 21.6 & 677.4 & $06-26-62$ & 362946116254101 \\
\hline
\end{tabular}

${ }^{1}$ Site numbers 1 through 5 are not shown on plate 1. 
Table 2. Ground-water levels and well data of wells located in the Death Valley Junction, California-Nevada, 30-minute $\times 60$-minute quadrangle [“--", no data available; $\mathrm{m}$, meter; $\mathrm{cm}$, centimeter; Height of measuring point.-- minus sign indicates measuring point above land surface; NWIS, National Water Information System]

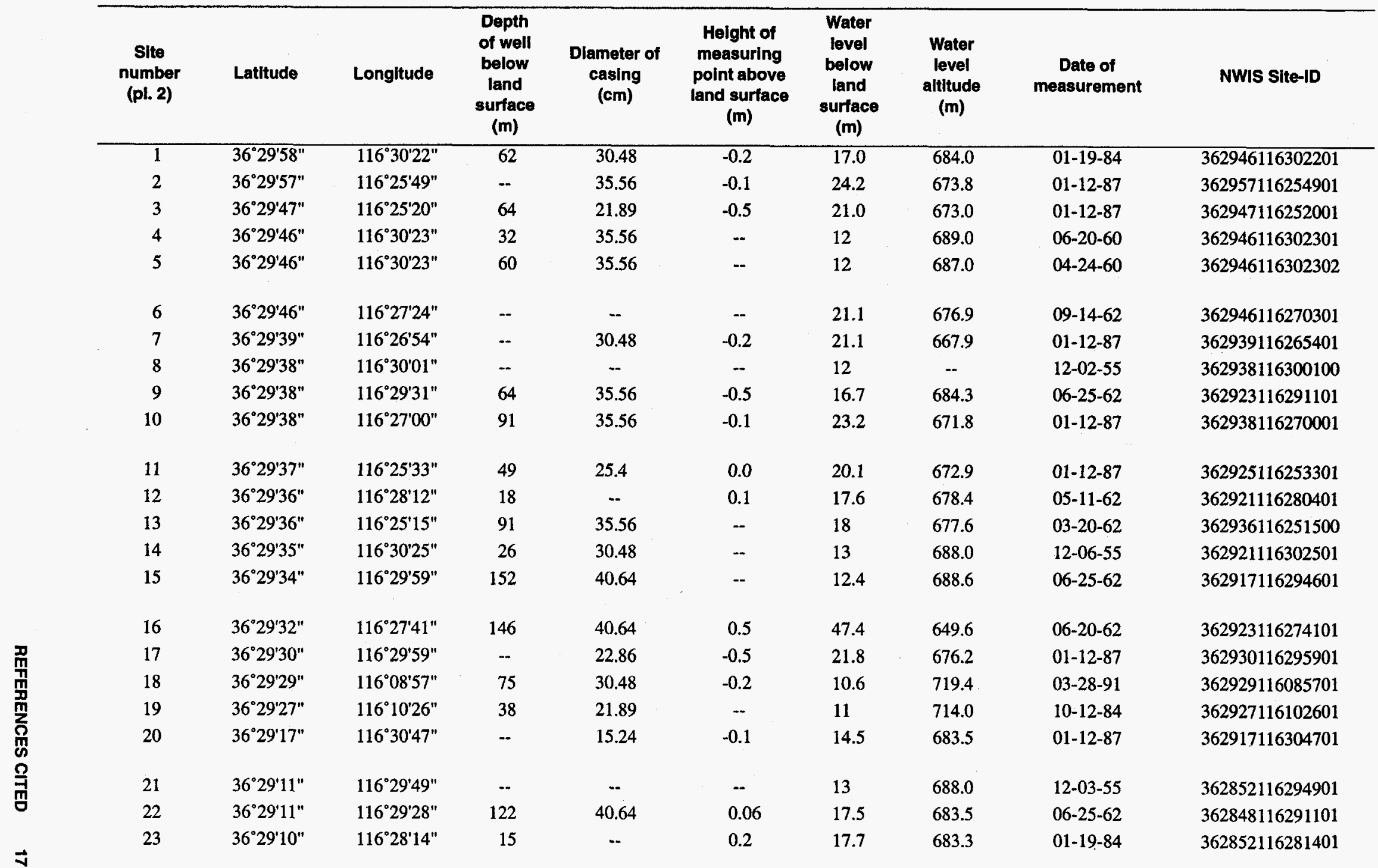


Table 2. Ground-water levels and well data of wells located in the Death Valley Junction, California-Nevada, 30-minute $\times 60$-minute quadrangle--Continued

\begin{tabular}{|c|c|c|c|c|c|c|c|c|c|}
\hline $\begin{array}{c}\text { Site } \\
\text { number } \\
\text { (pl. 2) }\end{array}$ & Latitude & Longltude & $\begin{array}{l}\text { Depth } \\
\text { of well } \\
\text { below } \\
\text { land } \\
\text { surface } \\
\text { (m) }\end{array}$ & $\begin{array}{l}\text { Diameter of } \\
\text { casing } \\
\text { (cm) }\end{array}$ & $\begin{array}{l}\text { Height of } \\
\text { measuring } \\
\text { point above } \\
\text { land surface } \\
\text { (m) }\end{array}$ & $\begin{array}{l}\text { Water } \\
\text { level } \\
\text { below } \\
\text { land } \\
\text { surface } \\
\text { (m) }\end{array}$ & $\begin{array}{l}\text { Water } \\
\text { level } \\
\text { altitude } \\
(\mathrm{m})\end{array}$ & $\begin{array}{l}\text { Date of } \\
\text { measurement }\end{array}$ & NWIS Site-1D \\
\hline 24 & $36^{\circ} 29^{\prime} 09^{\prime \prime}$ & $116^{\circ} 28^{\prime} 42^{\prime \prime}$ & 19 & - & 0.1 & 14.9 & 686.1 & $06-25-62$ & 362856116284201 \\
\hline 25 & $36^{\circ} 29^{\prime} 08^{\prime \prime}$ & $116^{\circ} 30^{\prime} 15^{\prime \prime}$ & 62 & 35.56 & -. & 15.5 & 685.5 & $10-31-62$ & 362848116301501 \\
\hline 26 & $36^{\circ} 29^{\prime} 05^{\prime \prime}$ & $116^{\circ} 27^{\prime} 40^{\prime \prime}$ & -- & 35.56 & -0.1 & 19.3 & 673.7 & $01-12-87$ & 362905116274001 \\
\hline 27 & $36^{\circ} 29^{\prime} 05^{\prime \prime}$ & $116^{\circ} 09^{\prime} 23^{\prime \prime}$ & 41 & 20.32 & -0.7 & 10.7 & 719.5 & 01-16-87 & 362905116092301 \\
\hline 28 & $36^{\circ} 29^{\prime} 02^{\prime \prime}$ & $116^{\circ} 30^{\prime} 47^{\prime \prime}$ & 62 & 30.48 & -0.2 & 21.6 & 674.4 & $01-12-87$ & 362902116304701 \\
\hline 29 & $36^{\circ} 28^{\prime} 58^{\prime \prime}$ & $116^{\circ} 19^{\prime} 53^{\prime \prime}$ & 73 & 30.48 & -- & 65.5 & 629.6 & 03-09-72 & 362858116195301 \\
\hline 30 & $36^{\circ} 28^{\prime} 58^{\prime \prime}$ & $116^{\circ} 09^{\prime} 15^{\prime \prime}$ & 26 & 40.64 & -- & 11 & 720.0 & $06-19-62$ & 362858116091502 \\
\hline 31 & $36^{\circ} 28^{\prime} 48^{\prime \prime}$ & $116^{\circ} 26^{\prime} 42^{\prime \prime}$ & 75 & 32.25 & -0.2 & 21.0 & 670.0 & $10-17-89$ & 362848116264201 \\
\hline 32 & $36^{\circ} 28^{\prime} 36^{\prime \prime}$ & $116^{\circ} 09^{\prime} 30^{\prime \prime}$ & 43 & 40.64 & -- & 10 & 719.0 & $10-30-61$ & 362858116091501 \\
\hline 33 & $36^{\circ} 28^{\prime} 35^{\prime \prime}$ & $116^{\circ} 26^{\prime} 41^{\prime \prime}$ & 61 & 25.4 & 0.0 & 20.8 & 668.2 & $04-10-90$ & 362835116264101 \\
\hline 34 & $36^{\circ} 27^{\prime} 40^{\prime \prime}$ & $116^{\circ} 11^{\prime} 26^{\prime \prime}$ & 7 & -. & -0.2 & 6.0 & 725.9 & $01-16-87$ & 362740116112601 \\
\hline 35 & $36^{\circ} 27^{\prime} 36^{\prime \prime}$ & $116^{\circ} 28^{\prime} 57^{\prime \prime}$ & 537 & 5.08 & -0.2 & 17.0 & 675.0 & $01-11-87$ & 362736116285701 \\
\hline 36 & $36^{\circ} 27^{\prime} 36^{\prime \prime}$ & $116^{\circ} 28^{\prime} 57^{\prime \prime}$ & 30 & 3.18 & -0.2 & 15.7 & 676.3 & $01-11-87$ & 362736116285702 \\
\hline 37 & $36^{\circ} 27^{\prime} 27^{\prime \prime}$ & $116^{\circ} 32^{\prime} 22^{\prime \prime}$ & 38 & 35.56 & -- & 13.0 & 671.0 & $06-19-62$ & 362727116322201 \\
\hline 38 & $36^{\circ} 27^{\prime} 26^{\prime \prime}$ & $116^{\circ} 31^{\prime} 44^{\prime \prime}$ & 91 & 35.56 & -0.4 & 11.2 & 671.8 & $01-13-87$ & 362726116314401 \\
\hline 39 & $36^{\circ} 27^{\prime} 25^{\prime \prime}$ & $116^{\circ} 30^{\prime} 59^{\prime \prime}$ & 120 & 35.56 & -0.1 & 10.0 & 672.0 & $01-13-87$ & 362725116305901 \\
\hline 40 & $36^{\circ} 27^{\prime} 15^{\prime \prime}$ & $116^{\circ} 32^{\prime} 23^{\prime \prime}$ & 91 & 35.56 & 0.0 & 9.8 & 675.2 & $08-03-86$ & 362715116322301 \\
\hline 41 & $36^{\circ} 27^{\prime} 11^{\prime \prime}$ & $116^{\circ} 49^{\prime} 44^{\prime \prime}$ & 76 & 35.56 & 0.4 & 23.2 & 122.8 & $01-23-86$ & 362711116494401 \\
\hline 42 & $36^{\circ} 27^{\prime} 05^{\prime \prime}$ & $116^{\circ} 32^{\prime} 23^{\prime \prime}$ &.- & 35.56 & 0.0 & 9.0 & 675.0 & $08-03-86$ & 362705116322301 \\
\hline 43 & $36^{\circ} 27^{\prime} 05^{\prime \prime}$ & $116^{\circ} 31^{\prime} 50^{\prime \prime}$ & -- & - & -0.2 & 9.4 & 673.6 & $01-11-86$ & 362705116315001 \\
\hline 44 & $36^{\circ} 27^{\prime} 05^{\prime \prime}$ & $116^{\circ} 30^{\prime} 09^{\prime \prime}$ & 7 & 152.40 & -. & 6.3 & 673.7 & $06-18-62$ & 362705116300901 \\
\hline 45 & $36^{\circ} 27^{\prime} 02^{\prime \prime}$ & $116^{\circ} 32^{\prime} 22^{\prime \prime}$ & 87 & 35.56 & -0.3 & 13.7 & 670.3 & 08-03-86 & 362702116322201 \\
\hline 46 & $36^{\circ} 26^{\prime} 50^{\prime \prime}$ & $116^{\circ} 31^{\prime} 15^{\prime \prime}$ & 91 & 35.56 & -- & 9.8 & 671.2 & $06-19-62$ & 362650116311501 \\
\hline
\end{tabular}


Table 2. Ground-water levels and well data of wells located in the Death Valley Junction, California-Nevada, 30-minute $\times 60$-minute quadrangle--Continued

\begin{tabular}{|c|c|c|c|c|c|c|c|c|c|}
\hline $\begin{array}{c}\text { Slie } \\
\text { number } \\
\text { (pl. 2) }\end{array}$ & Latitude & Longitude & $\begin{array}{l}\text { Depth } \\
\text { of well } \\
\text { below } \\
\text { land } \\
\text { surface } \\
\text { (m) }\end{array}$ & $\begin{array}{l}\text { Dlameter of } \\
\text { casing } \\
\text { (cm) }\end{array}$ & $\begin{array}{l}\text { Height of } \\
\text { measuring } \\
\text { point above } \\
\text { land surface } \\
\text { (m) }\end{array}$ & $\begin{array}{c}\text { Water } \\
\text { level } \\
\text { below } \\
\text { land } \\
\text { surface } \\
\text { (m) }\end{array}$ & $\begin{array}{l}\text { Water } \\
\text { level } \\
\text { altitude } \\
(\mathrm{m})\end{array}$ & $\begin{array}{c}\text { Date of } \\
\text { measurement }\end{array}$ & NWIS SIte-ID \\
\hline 47 & $36^{\circ} 26^{\prime} 48^{\prime \prime}$ & $116^{\circ} 27^{\circ} 46^{\prime \prime}$ & 46 & 15.24 & -0.1 & 15.6 & 673.4 & $01-15-87$ & 362648116274601 \\
\hline 48 & $36^{\circ} 26^{\prime} 42^{\prime \prime}$ & $116^{\circ} 21^{\prime} 25^{\prime \prime}$ & 157 & 40.64 & -- & Flows & Flows & $01-19-84$ & 362627116213501 \\
\hline 49 & $36^{\circ} 25^{\prime} 55^{\prime \prime}$ & $116^{\circ} 20^{\prime} 53^{\prime \prime}$ & -- & 25.4 & -0.3 & 2.30 & 657.7 & $03-17-87$ & 362555116205301 \\
\hline 50 & $36^{\circ} 25^{\prime} 54^{\prime \prime}$ & $116^{\circ} 20^{\prime} 40^{\prime \prime}$ & 37 & 21.89 & -- & 2 & 662.0 & $05-25-67$ & 362554116204001 \\
\hline 51 & $36^{\circ} 25^{\prime} 29^{\prime \prime}$ & $116^{\circ} 17^{\prime} 11^{\prime \prime}$ & 76 & 40.64 & -0.3 & 15.3 & -- & $09-07-89$ & 362529116171100 \\
\hline 52 & $36^{\circ} 25^{\prime} 29^{\prime \prime}$ & $116^{\circ} 16^{\prime} 05^{\prime \prime}$ & 81 & 32.25 & -0.4 & 22.7 & 719.3 & $07-25-89$ & 362529116160501 \\
\hline 53 & $36^{\circ} 25^{\prime} 28^{\prime \prime}$ & $116^{\circ} 21^{\prime} 25^{\prime \prime}$ & -- & 60.96 & -1.5 & .14 & 651.9 & $01-15-87$ & 362528116212501 \\
\hline 54 & $36^{\circ} 25^{\prime} 25^{\prime \prime}$ & $116^{\circ} 27^{\prime} 45^{\prime \prime}$ & 3 & -- & -- & 2.8 & 662.2 & $06-18-62$ & 362525116274501 \\
\hline 55 & $36^{\circ} 25^{\prime} 25^{\prime \prime}$ & $116^{\circ} 27^{\prime} 43^{\prime \prime}$ & 326 & 5.08 & -0.4 & 2.21 & 663.8 & $01-13-87$ & 362525116274301 \\
\hline 56 & $36^{\circ} 25^{\prime} 25^{\prime \prime}$ & $116^{\circ} 27^{\prime} 43^{\prime \prime}$ & 14 & 3.17 & -0.4 & 3.86 & 660.1 & $01-13-87$ & 362525116274302 \\
\hline 57 & $36^{\circ} 25^{\prime} 17^{\prime \prime}$ & $116^{\circ} 23^{\prime} 40^{\prime \prime}$ & 6 & 121.92 & 0.2 & 4.36 & 727.6 & $06-16-62$ & 362517116234001 \\
\hline 58 & $36^{\circ} 25^{\prime} 05^{\prime \prime}$ & $116^{\circ} 22^{\prime} 30^{\prime \prime}$ & 30 & 35.56 & -0.7 & 4.32 & 659.7 & $03-17-87$ & 362505116223001 \\
\hline 59 & $36^{\circ} 24^{\prime} 52^{\prime \prime}$ & $116^{\circ} 25^{\prime} 10^{\prime \prime}$ & 123 & 32.25 & 0.03 & 23.5 & 641.5 & $01-18-84$ & 362444116251001 \\
\hline 60 & $36^{\circ} 24^{\prime} 43^{\prime \prime}$ & $116^{\circ} 26^{\prime} 36^{\prime \prime}$ & -. & 35.56 & -0.5 & 7.59 & 650.4 & $01-13-87$ & 362443116263602 \\
\hline 61 & $36^{\circ} 24^{\prime} 32^{\prime \prime}$ & $116^{\circ} 16^{\prime} 57^{\prime \prime}$ & 152 & 40.64 & 0.0 & 6.68 & 700.3 & 07-25-89 & 362432116165701 \\
\hline 62 & $36^{\circ} 24^{\prime} 25^{\prime \prime}$ & $116^{\circ} 18^{\prime} 10^{\prime \prime}$ & 85 & -- & -0.1 & 6.14 & 677.9 & $03-17-87$ & 362425116181001 \\
\hline 63 & $36^{\circ} 24^{\prime} 25^{\prime \prime}$ & $116^{\circ} 16^{\prime} 49^{\prime \prime}$ & 62 & 16.81 & -- & 8 & 696.0 & $07-08-68$ & 362425116164901 \\
\hline 64 & $36^{\circ} 24^{\prime} 15^{\prime \prime}$ & $116^{\circ} 27^{\prime} 05^{\prime \prime}$ &.- & 16.84 & -0.5 & 9.20 & 650.8 & $01-13-87$ & 362415116270501 \\
\hline 65 & $36^{\circ} 24^{\prime} 12^{\prime \prime}$ & $116^{\circ} 15^{\prime} 45^{\prime \prime}$ & -- & .. & - & 20.5 & 692.5 & $03-09-72$ & 362412116154501 \\
\hline 66 & $36^{\circ} 24^{\prime} 11^{\prime \prime}$ & $116^{\circ} 16^{\prime} 33^{\prime \prime}$ & 143 & 35.56 & -0.2 & 3.5 & 700.5 & $12-01-88$ & 362417116163600 \\
\hline 67 & $36^{\circ} 24^{\prime} 10^{\prime \prime}$ & $116^{\circ} 16^{\prime} 09^{\prime \prime}$ & 120 & 40.64 & -0.2 & 1.94 & 705.1 & $01-15-87$ & 362410116160901 \\
\hline 68 & $36^{\circ} 24^{\prime} 09^{\prime \prime}$ & $116^{\circ} 15^{\prime} 56^{\prime \prime}$ & 126 & 40.64 & -0.8 & .65 & 703.3 & $03-17-87$ & 362409116155601 \\
\hline 69 & $36^{\circ} 24^{\prime} 08^{\prime \prime}$ & $116^{\circ} 15^{\prime} 40^{\prime \prime}$ & 196 & 40.64 & 0.0 & 4.67 & 705.3 & $03-17-87$ & 362408116154001 \\
\hline 70 & $36^{\circ} 24^{\prime} 06^{\prime \prime}$ & $116^{\circ} 15^{\prime} 40^{\prime \prime}$ & 152 & 40.64 & -0.2 & 2.88 & 713.1 & $03-17-87$ & 362406116154001 \\
\hline
\end{tabular}




\begin{tabular}{|c|c|c|c|c|c|c|c|c|c|}
\hline $\begin{array}{c}\text { Site } \\
\text { number } \\
\text { (pl. 2) }\end{array}$ & Latitude & Longitude & $\begin{array}{l}\text { Depth } \\
\text { of well } \\
\text { below } \\
\text { land } \\
\text { surface } \\
\text { (m) }\end{array}$ & $\begin{array}{l}\text { Diameter of } \\
\text { casing } \\
\text { (cm) }\end{array}$ & $\begin{array}{l}\text { Height of } \\
\text { measuring } \\
\text { point above } \\
\text { land surface } \\
\text { (m) }\end{array}$ & $\begin{array}{l}\text { Water } \\
\text { level } \\
\text { below } \\
\text { land } \\
\text { surface } \\
\text { (m) }\end{array}$ & $\begin{array}{c}\text { Water } \\
\text { level } \\
\text { altitude } \\
\text { (m) }\end{array}$ & $\begin{array}{c}\text { Date of } \\
\text { measurement }\end{array}$ & NWIS Site-ID \\
\hline 71 & $36^{\circ} 24^{\prime} 03^{\prime \prime}$ & $116^{\circ} 16^{\prime} 08^{\prime \prime}$ & 86 & 37.33 & -- & 5.65 & 698.3 & $10-29-70$ & 362403116160801 \\
\hline 72 & $36^{\circ} 23^{\prime} 58^{\prime \prime}$ & $116^{\circ} 16^{\prime} 33^{\prime \prime}$ & 152 & 40.64 & -- & Flows & Flows & $03-17-87$ & 362358116163301 \\
\hline 73 & $36^{\circ} 23^{\prime} 58^{\prime \prime}$ & $116^{\circ} 16^{\prime} 01^{\prime \prime}$ & -- & -- & -- & 4.6 & 699.7 & $02-03-71$ & 362358116160102 \\
\hline 74 & $36^{\circ} 23^{\prime} 14^{\prime \prime}$ & $116^{\circ} 16^{\prime} 01^{\prime \prime}$ & 46 & 21.89 & -- & Flows & Flows & $01-17-87$ & 362314116160101 \\
\hline 75 & $36^{\circ} 22^{\prime} 50^{\prime \prime}$ & $116^{\circ} 26^{\prime} 51^{\prime \prime}$ & 512 & 5.08 & -0.6 & 23 & 641.0 & $10-02-87$ & 362250116265101 \\
\hline 76 & $36^{\circ} 22^{\prime} 50^{\prime \prime}$ & $116^{\circ} 26^{\prime} 51^{\prime \prime}$ & 38 & 3.17 & -0.6 & 23 & 641.0 & $10-02-87$ & 362250116265102 \\
\hline 77 & $36^{\circ} 22^{\prime} 50^{\prime \prime}$ & $116^{\circ} 17^{\prime} 53^{\prime \prime}$ & 30 & 16.81 & 0.0 & 1.63 & 673.4 & $03-17-87$ & 362250116175301 \\
\hline 78 & $36^{\circ} 21^{\prime} 54^{\prime \prime}$ & $116^{\circ} 16^{\prime} 20^{\prime \prime}$ & 81 & 21.89 & -0.6 & .88 & 672.1 & $01-14-87$ & 362154116162001 \\
\hline 79 & $36^{\circ} 21^{\prime} 40^{\prime \prime}$ & $116^{\circ} 26^{\prime} 10^{\prime \prime}$ & 604 & 5.08 & -0.3 & 21.2 & 624.8 & $01-13-87$ & 362140116261001 \\
\hline 80 & $36^{\circ} 21^{\prime} 31^{\prime \prime}$ & $116^{\circ} 16^{\prime} 01^{\prime \prime}$ & 37 & 3.17 & -0.5 & 3.86 & 688.1 & $01-14-87$ & 362113116160102 \\
\hline 81 & $36^{\circ} 21^{\prime} 13^{\prime \prime}$ & $116^{\circ} 16^{\prime} 01^{\prime \prime}$ & 365 & 5.08 & -0.5 & 4.38 & 687.6 & $01-14-87$ & 362113116160101 \\
\hline 83 & $36^{\circ} 20^{\prime} 54^{\prime \prime}$ & $116^{\circ} 01^{\prime} 34^{\prime \prime}$ & 131 & 20.32 & $\ldots$ & 111 & 786.0 & $06-01-71$ & 360256116013501 \\
\hline 84 & $36^{\circ} 20^{\prime} 36^{\prime \prime}$ & $116^{\circ} 01^{\prime} 50^{\prime \prime}$ & 128 & 20.95 & 0.34 & 92.1 & 776.9 & $02-17-76$ & 362036116015001 \\
\hline 85 & $36^{\circ} 20^{\prime} 33^{\prime \prime}$ & $116^{\circ} 25^{\prime} 52^{\prime \prime}$ & -- & 16.84 & -0.2 & 11.3 & 627.7 & $01-13-87$ & 362033116255202 \\
\hline 86 & $36^{\circ} 20^{\prime} 14^{\prime \prime}$ & $116^{\circ} 13^{\prime} 49^{\prime \prime}$ & 37 & 3.17 & -0.7 & 24.8 & 716.2 & $01-14-87$ & 362014116134902 \\
\hline 87 & $36^{\circ} 20^{\prime} 14^{\prime \prime}$ & $116^{\circ} 13^{\prime} 39^{\prime \prime}$ & 482 & 5.08 & -0.6 & 26.8 & 714.2 & $01-14-87$ & 362014116133901 \\
\hline 88 & $36^{\circ} 19^{\prime} 54^{\prime \prime}$ & $116^{\circ} 18^{\prime} 12^{\prime \prime}$ & 610 & 5.08 & -0.3 & 64.0 & 632.0 & $01-14-87$ & 361954116181201 \\
\hline 89 & $36^{\circ} 19^{\prime} 54^{\prime \prime}$ & $116^{\circ} 18^{\prime} 12^{\prime \prime}$ & 49 & 3.17 & -0.3 & 25.5 & 670.5 & $01-14-87$ & 361954116181202 \\
\hline 90 & $36^{\circ} 19^{\prime} 09^{\prime \prime}$ & $116^{\circ} 01^{\prime} 34^{\prime \prime}$ & 227 & 40.64 & 1.01 & 33.7 & 778.3 & $02-25-75$ & 361909116013401 \\
\hline 91 & $36^{\circ} 19^{\prime} 08^{\prime \prime}$ & $116^{\circ} 01^{\prime} 34^{\prime \prime}$ & -- & -- & 0.15 & 36.4 & 774.6 & $02-17-76$ & 361908116013401 \\
\hline 92 & $36^{\circ} 19^{\prime} 07^{\prime \prime}$ & $116^{\circ} 01^{\prime} 35^{\prime \prime}$ & -- & -- & -- & 33.6 & 776.4 & 03-08-77 & 361907116013501 \\
\hline 93 & $36^{\circ} 19^{\prime} 03^{\prime \prime}$ & $116^{\circ} 01^{\prime 2} 20^{\prime \prime}$ & 235 & -- & -- & 35.3 & 776.7 & $02-17-76$ & 361903116012001 \\
\hline 94 & $36^{\circ} 19^{\prime} 00^{\prime \prime}$ & $116^{\circ} 03^{\prime} 08^{\prime \prime}$ & 46 & 20.32 & 0.06 & 34.1 & 775.9 & $02-17-76$ & 361900116030801 \\
\hline 95 & $36^{\circ} 18^{\prime} 57^{\prime \prime}$ & $116^{\circ} 02^{\prime} 31^{\prime \prime}$ & 143 & 40.64 & 0.0 & 31.8 & 776.2 & $02-17-76$ & 361857116023101 \\
\hline
\end{tabular}


Table 2. Ground-water levels and well data of wells located in the Death Valley Junction, California-Nevada, 30-minute $\times 60$-minute quadrangle--Continued

\begin{tabular}{|c|c|c|c|c|c|c|c|c|c|}
\hline $\begin{array}{c}\text { Site } \\
\text { number } \\
\text { (pl. 2) }\end{array}$ & Latitude & Longitude & $\begin{array}{l}\text { Depth } \\
\text { of well } \\
\text { below } \\
\text { land } \\
\text { surface } \\
\text { (m) }\end{array}$ & $\begin{array}{l}\text { Diameter of } \\
\text { casing } \\
\text { (cm) }\end{array}$ & $\begin{array}{l}\text { Height of } \\
\text { measuring } \\
\text { point above } \\
\text { land surface } \\
\text { (m) }\end{array}$ & $\begin{array}{l}\text { Water } \\
\text { level } \\
\text { below } \\
\text { land } \\
\text { surface } \\
\text { (m) }\end{array}$ & $\begin{array}{l}\text { Water } \\
\text { level } \\
\text { altitude } \\
\text { (m) }\end{array}$ & $\begin{array}{c}\text { Date of } \\
\text { measurement }\end{array}$ & NWIS Site-1D \\
\hline 96 & $36^{\circ} 18^{\prime} 53^{\prime \prime}$ & $116^{\circ} 01^{\prime} 56^{\prime \prime}$ & 114 & 40.64 & 0.43 & 31.6 & 777.4 & $02-25-75$ & 361853116015601 \\
\hline 97 & $36^{\circ} 18^{\prime} 40^{\prime \prime}$ & $116^{\circ} 18^{\prime} 40^{\prime \prime}$ & -- & 11.43 & 0.0 & 29.4 & 674.6 & $05-18-84$ & 361840116184001 \\
\hline 98 & $36^{\circ} 18^{\prime} 40^{\prime \prime}$ & $116^{\circ} 18^{\prime} 40^{\prime \prime}$ & -- & 10.16 & -0.5 & 27.9 & 676.1 & $05-18-84$ & 361840116184002 \\
\hline 99 & $36^{\circ} 18^{\prime} 40^{\prime \prime}$ & $116^{\circ} 18^{\prime} 40^{\prime \prime}$ & -- & 11.43 & -0.1 & 29.6 & 674.4 & $05-18-84$ & 361840116184003 \\
\hline 100 & $36^{\circ} 18^{\prime} 40^{\prime \prime}$ & $116^{\circ} 18^{\prime} 40^{\prime \prime}$ & -- & 5.08 & -0.2 & 29.8 & 674.2 & $05-18-84$ & 361840116184004 \\
\hline 101 & $36^{\circ} 18^{\prime} 40^{\prime \prime}$ & $116^{\circ} 18^{\prime} 40^{\prime \prime}$ & -. & 21.59 & -0.4 & 31.2 & 672.8 & $05-18-84$ & 361840116184005 \\
\hline 102 & $36^{\circ} 18^{\prime} 40^{\prime \prime}$ & $116^{\circ} 18^{\prime} 40^{\prime \prime}$ & $\cdots$ & 5.08 & -0.3 & 33.2 & 670.8 & $05-18-84$ & 361840116184006 \\
\hline 103 & $36^{\circ} 18^{\prime} 40^{\prime \prime}$ & $116^{\circ} 18^{\circ} 40^{\prime \prime}$ & -- & 16.17 & 0.0 & 32.5 & 671.5 & $05-18-84$ & 361840116184007 \\
\hline 104 & $36^{\circ} 18^{\prime} 35^{\prime \prime}$ & $116^{\circ} 22^{\prime} 45^{\prime \prime}$ & 2 & 76.20 & - & 1.4 & 619.6 & $06-19-62$ & 361835116224501 \\
\hline 106 & $36^{\circ} 18^{\prime} 26^{\prime \prime}$ & $116^{\circ} 02^{\prime} 28^{\prime \prime}$ & 139 & 40.64 & 0.03 & 26.7 & 776.3 & $02-17-76$ & 361826116022801 \\
\hline 107 & $36^{\circ} 18^{\prime} 20^{\prime \prime}$ & $116^{\circ} 25^{\prime} 13^{\prime \prime}$ & 49 & 20.32 & -0.3 & 2.65 & 622.3 & $03-18-87$ & 361820116251301 \\
\hline 108 & $36^{\circ} 18^{\prime} 17^{\prime \prime}$ & $116^{\circ} 24^{\prime} 47^{\prime \prime}$ & 61 & 30.48 & 0.49 & 1.51 & 619.5 & $01-20-84$ & 361817116244701 \\
\hline 109 & $36^{\circ} 18^{\prime} 14^{\prime \prime}$ & $116^{\circ} 34^{\prime} 57^{\prime \prime}$ & -- & -- & -- & 76 & 826.0 & $03-26-87$ & 361814116345701 \\
\hline 110 & $36^{\circ} 18^{\prime} 08^{\prime \prime}$ & $116^{\circ} 27^{\prime} 35^{\prime \prime}$ & 41 & 25.4 & 0.0 & 39.5 & 628.5 & $03-18-87$ & 361808116273501 \\
\hline 111 & $36^{\circ} 18^{\prime} 02^{\prime \prime}$ & $116^{\circ} 00^{\prime} 16^{\prime \prime}$ & 120 & -- & -- & 32.4 & 779.6 & $02-17-76$ & 361802116001601 \\
\hline 112 & $36^{\circ} 18^{\prime} 01^{\prime \prime}$ & $116^{\circ} 00^{\prime} 17^{\prime \prime}$ & 46 & 20.32 & 0.03 & 31.6 & 779.4 & $02-17-76$ & 361801116001701 \\
\hline 113 & $36^{\circ} 18^{\prime} 00^{\prime \prime}$ & $116^{\circ} 24^{\prime} 50^{\prime \prime}$ & 42 & 30.48 & -- & .7 & 620.3 & $06-19-62$ & 361800116245001 \\
\hline 114 & $36^{\circ} 18^{\prime} 00^{\prime \prime}$ & $=116^{\circ} 24^{\prime} 43^{\prime \prime}$ & 21 & 30.48 & 0.0 & 1.10 & 619.9 & $01-14-87$ & 361800116244301 \\
\hline 115 & $36^{\circ} 17^{\prime} 53^{\prime \prime}$ & $116^{\circ} 00^{\prime} 09^{\prime \prime}$ & 120 & 40.64 & -0.3 & 32.9 & 780.1 & $03-27-91$ & 361753116000901 \\
\hline 116 & $36^{\circ} 17^{\circ} 45^{\prime \prime}$ & $116^{\circ} 21^{\prime} 55^{\prime \prime}$ & 2 & 15.24 & -- & .5 & 617.5 & $06-21-62$ & 361745116215502 \\
\hline 117 & $36^{\circ} 17^{\prime} 41^{\prime \prime}$ & $116^{\circ} 00^{\prime} 10^{\prime \prime}$ & 315 & 40.64 & 0.09 & 31.5 & 779.5 & $02-17-76$ & 361741116001001 \\
\hline 118 & $36^{\circ} 17^{\prime} 34^{\prime \prime}$ & $116^{\circ} 01^{\prime} 22^{\prime \prime}$ & 214 & - & 0.0 & 21.5 & 777.5 & $02-17-76$ & 361734116012201 \\
\hline
\end{tabular}


Table 2. Ground-water levels and well data of wells located in the Death Valley Junction, California-Nevada, 30-minute $\times 60$-minute quadrangle--Continued

\begin{tabular}{|c|c|c|c|c|c|c|c|c|c|}
\hline $\begin{array}{c}\text { Site } \\
\text { number } \\
\text { (pl. 2) }\end{array}$ & Latitude & Longitude & $\begin{array}{l}\text { Depth } \\
\text { of well } \\
\text { below } \\
\text { land } \\
\text { surface } \\
\text { (m) }\end{array}$ & $\begin{array}{l}\text { Diameter of } \\
\text { casing } \\
\text { (cm) }\end{array}$ & $\begin{array}{l}\text { Height of } \\
\text { measuring } \\
\text { point above } \\
\text { land surface } \\
\text { (m) }\end{array}$ & $\begin{array}{l}\text { Water } \\
\text { level } \\
\text { below } \\
\text { land } \\
\text { surface } \\
\text { (m) }\end{array}$ & $\begin{array}{l}\text { Water } \\
\text { level } \\
\text { altitude } \\
\text { (m) }\end{array}$ & $\begin{array}{c}\text { Date of } \\
\text { measurement }\end{array}$ & NWIS Site-ID \\
\hline 119 & $36^{\circ} 17^{\prime 2} 29^{\prime \prime}$ & $116^{\circ} 00^{\prime} 55^{\prime \prime}$ & 246 & -- & 0.15 & 24.9 & 779.1 & $03-17-76$ & 361735116500001 \\
\hline 120 & $36^{\circ} 17^{\prime} 24^{\prime \prime}$ & $116^{\circ} 32^{\prime} 42^{\prime \prime}$ & 610 & 5.08 & -0.4 & 114 & 706.0 & $01-13-87$ & 361724116324201 \\
\hline 121 & $36^{\circ} 17^{\prime} 24^{\prime \prime}$ & $116^{\circ} 32^{\prime} 42^{\prime \prime}$ & 137 & 3.17 & -0.5 & 121 & 699.0 & $01-13-87$ & 361724116324202 \\
\hline 122 & $36^{\circ} 17^{\prime} 23^{\prime \prime}$ & $116^{\circ} 00^{\prime} 03^{\prime \prime}$ & 165 & 40.64 & 0.37 & 30.9 & 780.1 & $02-17-76$ & 361723116000301 \\
\hline 123 & $36^{\circ} 17^{\prime} 09^{\prime \prime}$ & $116^{\circ} 01^{\prime} 24^{\prime \prime}$ & 107 & -- & 0.06 & 20.6 & 775.4 & $02-16-76$ & 361709116012401 \\
\hline 124 & $36^{\circ} 17^{\prime} 09^{\prime \prime}$ & $116^{\circ} 00^{\prime} 51^{\prime \prime}$ & 183 & 40.64 & -- & 13.4 & 786.6 & $06-18-55$ & 361709116005101 \\
\hline 125 & $36^{\circ} 17^{\prime} 09^{\prime \prime}$ & $116^{\circ} 00^{\prime} 42^{\prime \prime}$ & 145 & 40.64 & - & 20.4 & 781.6 & $02-04-62$ & 361709116004201 \\
\hline 126 & $36^{\circ} 17^{\prime} 05^{\prime \prime}$ & $116^{\circ} 21^{\prime} 35^{\prime \prime}$ & 2 & 30.48 & -. & -1.2 & 615.2 & $08-24-62$ & 361705116213501 \\
\hline 127 & $36^{\circ} 17^{\prime} 04^{\prime \prime}$ & $116^{\circ} 03^{\prime} 03^{\prime \prime}$ & - & - & 0.09 & 12.0 & 775.0 & $02-18-76$ & 361704116030301 \\
\hline 129 & $36^{\circ} 17^{\prime} 00^{\prime \prime}$ & $116^{\circ} 22^{\prime} 02^{\prime \prime}$ & 5 & 2.54 & -1.1 & -.62 & 617.6 & $07-09-86$ & 361700116220203 \\
\hline 130 & $36^{\circ} 17^{\prime} 00^{\prime \prime}$ & $116^{\circ} 22^{\prime} 02^{\prime \prime}$ & 8.9 & 5.08 & -1.0 & -2.28 & 617.3 & $06-24-83$ & 361727116170900 \\
\hline 131 & $36^{\circ} 17^{\prime} 00^{\prime \prime}$ & $116^{\circ} 22^{\prime} 02^{\prime \prime}$ & 2 & 2.54 & -1.3 & .85 & 613.1 & $07-09-86$ & 361700116220204 \\
\hline 132 & $36^{\circ} 16^{\prime} 47^{\prime \prime}$ & $116^{\circ} 04^{\prime} 39^{\prime \prime}$ & 19 & -- & - & 10.5 & 774.5 & $03-08-77$ & 361647116043901 \\
\hline 134 & $36^{\circ} 16^{\prime} 42^{\prime \prime}$ & $116^{\circ} 01^{\prime} 23^{\prime \prime}$ & 152 & 40.64 & -- & 14.6 & 781.4 & $04-03-61$ & 361642116012301 \\
\hline 135 & $36^{\circ} 16^{\prime} 40^{\prime \prime}$ & $116^{\circ} 31^{\prime} 55^{\prime \prime}$ & 3 & 30.48 & -. & -.2 & 613.2 & $08-24-62$ & 361640116315501 \\
\hline 136 & $36^{\circ} 16^{\prime} 38^{\prime \prime}$ & $116^{\circ} 04^{\prime} 37^{\prime \prime}$ & 10 & 3.17 & - & 6.49 & 778.5 & $02-04-62$ & 361638116043701 \\
\hline 137 & $36^{\circ} 16^{\prime} 36^{\prime \prime}$ & $116^{\circ} 00^{\prime} 09^{\prime \prime}$ & 336 & 40.64 & -- & 6.93 & 799.1 & $11-26-52$ & 361636116000901 \\
\hline 139 & $36^{\circ} 16^{\prime} 27^{\prime \prime}$ & $116^{\circ} 22^{\prime} 12^{\prime \prime}$ & 8.8 & 5.08 & -0.4 & .95 & 612.0 & $07-09-86$ & 361635116172100 \\
\hline 140 & $36^{\circ} 16^{\prime} 27^{\prime \prime}$ & $116^{\circ} 22^{\prime} 12^{\prime \prime}$ & 8.3 & 2.54 & -2.1 & -1.64 & 614.6 & $07-09-86$ & 361627116221201 \\
\hline 141 & $36^{\circ} 16^{\prime} 27^{\prime \prime}$ & $116^{\circ} 22^{\prime} 12^{\prime \prime}$ & 6.4 & 2.54 & -0.9 & -.10 & 613.1 & $07-09-86$ & 361627116221202 \\
\hline 142 & $36^{\circ} 16^{\prime} 16^{\prime \prime}$ & $116^{\circ} 01^{\prime} 25^{\prime \prime}$ & 61 & -. & -- & 12.9 & 782.1 & $02-04-62$ & 361616116012501 \\
\hline
\end{tabular}


Table 2. Ground-water levels and well data of wells located in the Death Valley Junction, California-Nevada, 30-minute $\times 60$-minute quadrangle--Continued

\begin{tabular}{|c|c|c|c|c|c|c|c|c|c|c|}
\hline & $\begin{array}{c}\text { Site } \\
\text { number } \\
\text { (pl. 2) }\end{array}$ & Latitude & Longitude & $\begin{array}{l}\text { Depth } \\
\text { of well } \\
\text { below } \\
\text { land } \\
\text { surface } \\
\text { (m) }\end{array}$ & $\begin{array}{l}\text { Dlameter of } \\
\text { casing } \\
\text { (cm) }\end{array}$ & $\begin{array}{l}\text { Height of } \\
\text { measuring } \\
\text { point above } \\
\text { land surface } \\
\text { (m) }\end{array}$ & $\begin{array}{l}\text { Water } \\
\text { level } \\
\text { below } \\
\text { land } \\
\text { surface } \\
\text { (m) }\end{array}$ & $\begin{array}{l}\text { Water } \\
\text { level } \\
\text { altitude } \\
\text { (m) }\end{array}$ & $\begin{array}{l}\text { Date of } \\
\text { measurement }\end{array}$ & NWIS Site-ID \\
\hline & 145 & $36^{\circ} 16^{\prime} 12^{\prime \prime}$ & $116^{\circ} 00^{\prime} 51^{\prime \prime}$ & 155 & 40.64 & -- & 19.4 & 788.6 & $02-17-76$ & 361612116005101 \\
\hline & 146 & $36^{\circ} 16^{\prime} 10^{\prime \prime}$ & $116^{\circ} 22^{\prime} 37^{\prime \prime}$ & 7 & 5.08 & -0.9 & 3.99 & 608.0 & $03-22-86$ & 361610116223701 \\
\hline & 147 & $36^{\circ} 16^{\prime} 08^{\prime \prime}$ & $116^{\circ} 04^{\prime} 41^{\prime \prime}$ & 155 & 12.0 & 0.4 & 9.67 & 773.3 & $02-17-76$ & 361608116044101 \\
\hline & 148 & $36^{\circ} 16^{\prime} 06^{\prime \prime}$ & $116^{\circ} 21^{\prime} 27^{\prime \prime}$ & 4 & -- & -1.2 & .49 & 611.5 & $03-21-86$ & 361605116212702 \\
\hline & 149 & $36^{\circ} 16^{\prime} 05^{\prime \prime}$ & $116^{\circ} 31^{\prime} 48^{\prime \prime}$ & -- & -- & -- & 31.3 & 735.7 & $01-09-86$ & 361605116314801 \\
\hline & 150 & $36^{\circ} 16^{\prime} 05^{\prime \prime}$ & $116^{\circ} 21^{\prime} 27^{\prime \prime}$ & 2 & -- & -1.2 & .55 & 611.4 & $03-21-86$ & 361605116212701 \\
\hline & 153 & $36^{\circ} 15^{\prime} 53^{\prime \prime}$ & $116^{\circ} 21^{\prime} 21^{\prime \prime}$ & 6.8 & -. & -1.1 & 1.19 & 609.8 & $07-09-86$ & 361553116212100 \\
\hline & 154 & $36^{\circ} 15^{\prime} 53^{\prime \prime}$ & $116^{\circ} 21^{\prime} 21^{\prime \prime}$ & 6.5 & 5.08 & -0.8 & .23 & 610.8 & $07-09-86$ & 361553116212101 \\
\hline & 155 & $36^{\circ} 15^{\prime} 53^{\prime \prime}$ & $116^{\circ} 21^{\prime} 21^{\prime \prime}$ & 1.6 & 5.08 & -1.5 & 1.23 & 609.8 & $07-09-86$ & 361553116212102 \\
\hline & 156 & $36^{\circ} 15153^{\prime \prime}$ & $116^{\circ} 21^{\prime} 21^{\prime \prime}$ & 8 & 2.54 & -1.0 & -.49 & 612.5 & $07-09-86$ & 361553116212103 \\
\hline & 157 & $36^{\circ} 15^{\prime} 53^{\prime \prime}$ & $116^{\circ} 21^{\prime} 21^{\prime \prime}$ & 10 & -- & -1.0 & -.31 & 611.3 & $07-09-86$ & 361553116212104 \\
\hline & 158 & $36^{\circ} 15^{\prime} 52^{\prime \prime}$ & $116^{\circ} 00^{\prime} 09^{\prime \prime}$ & 261 & 18.0 & - & 18.9 & 784.1 & $02-17-76$ & 361552116000901 \\
\hline & 159 & $36^{\circ} 15^{\prime} 48^{\prime \prime}$ & $116^{\circ} 01^{\prime} 45^{\prime \prime}$ & 154 & 12.0 & -- & 10.7 & 777.3 & $02-18-76$ & 361548116014501 \\
\hline & 160 & $36^{\circ} 15^{\prime} 48^{\prime \prime}$ & $116^{\circ} 00^{\prime} 54^{\prime \prime}$ & 157 & 40.64 & - & 16.5 & 780.5 & $02-18-76$ & 361548116005401 \\
\hline & 161 & $36^{\circ} 15^{\prime} 36^{\prime \prime}$ & $116^{\circ} 00^{\prime} 51^{\prime \prime}$ & 236 & 40.64 & -- & 14.9 & 780.1 & $03-18-76$ & 361536116005101 \\
\hline \multirow{9}{*}{ 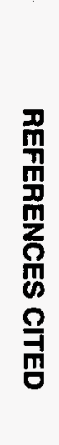 } & 162 & $36^{\circ} 15^{\prime} 33^{\prime \prime}$ & $116^{\circ} 11^{\prime} 19^{\prime \prime}$ & 8.2 & 3.17 & 0.79 & 6.22 & 742.8 & $01-28-76$ & 361533116111901 \\
\hline & 163 & $36^{\circ} 15^{\prime} 30^{\prime \prime}$ & $116^{\circ} 03^{\prime} 01^{\prime \prime}$ & -- & -- & -- & 8.28 & 773.7 & $02-18-76$ & 361530116030101 \\
\hline & 164 & $36^{\circ} 15^{\prime} 25^{\prime \prime}$ & $116^{\circ} 00^{\prime} 18^{\prime \prime}$ & 217 & 40.64 & - & .00 & 799.0 & -- & 361525116001801 \\
\hline & 165 & $36^{\circ} 15^{\prime} 23^{\prime \prime}$ & $116^{\circ} 01^{\prime} 56^{\prime \prime}$ & 262 & 40.64 & -- & 6.24 & 781.8 & $02-04-62$ & 361523116015601 \\
\hline & 166 & $36^{\circ} 15^{\prime} 23^{\prime \prime}$ & $116^{\circ} 00^{\prime} 51^{\prime \prime}$ & 236 & 12.0 & -- & 16.0 & 778.8 & $07-02-85$ & 361523116005101 \\
\hline & 167 & $36^{\circ} 15^{\prime} 22^{\prime \prime}$ & $116^{\circ} 04^{\prime} 47^{\prime \prime}$ & 152 & 10.0 & 0.37 & 8.70 & 773.3 & $02-18-76$ & 361522116044701 \\
\hline & 168 & $36^{\circ} 15^{\prime} 22^{\prime \prime}$ & $116^{\circ} 01^{\prime 2} 23^{\prime \prime}$ & 85 & 12.0 & -- & 10.7 & 779.3 & $02-20-73$ & 361522116012301 \\
\hline & 169 & $36^{\circ} 15^{\prime} 17^{\prime \prime}$ & $116^{\circ} 32^{\prime} 24^{\prime \prime}$ & -- & 6.5 & -0.2 & 20.0 & 833.0 & $01-09-86$ & 361517116322401 \\
\hline & 170 & $36^{\circ} 15^{\prime} 16^{\prime \prime}$ & $116^{\circ} 22^{\prime} 01^{\prime \prime}$ & 6.9 & 5.08 & -0.9 & 1.94 & 609.1 & $07-10-86$ & 361527116171300 \\
\hline
\end{tabular}


Table 2. Ground-water levels and well data of wells located in the Death Valley Junction, California-Nevada, 30-minute $\times 60$-minute quadrangle--Continued

\begin{tabular}{|c|c|c|c|c|c|c|c|c|c|}
\hline $\begin{array}{c}\text { Site } \\
\text { number } \\
\text { (pl. 2) }\end{array}$ & Latitude & Longitude & $\begin{array}{l}\text { Depth } \\
\text { of well } \\
\text { below } \\
\text { land } \\
\text { surface } \\
\text { (m) }\end{array}$ & $\begin{array}{l}\text { Diameter of } \\
\text { casing } \\
\text { (cm) }\end{array}$ & $\begin{array}{l}\text { Height of } \\
\text { measuring } \\
\text { point above } \\
\text { land surface } \\
\text { (m) }\end{array}$ & $\begin{array}{l}\text { Water } \\
\text { level } \\
\text { below } \\
\text { land } \\
\text { surface } \\
\text { (m) }\end{array}$ & $\begin{array}{c}\text { Water } \\
\text { level } \\
\text { altitude } \\
\text { (m) }\end{array}$ & $\begin{array}{l}\text { Date of } \\
\text { measurement }\end{array}$ & NWIS SIte-1D \\
\hline 171 & $36^{\circ} 15^{\prime} 16^{\prime \prime}$ & $116^{\circ} 22^{\prime} 01^{\prime \prime}$ & 3.3 & 2.54 & -1.2 & 2.03 & 609.0 & $07-10-86$ & 361516116220101 \\
\hline 172 & $36^{\circ} 15^{\prime} 16^{\prime \prime}$ & $116^{\circ} 22^{\prime} 01^{\prime \prime}$ & 10 & 2.54 & -0.9 & 1.88 & 609.1 & $07-10-86$ & 361516116220102 \\
\hline 173 & $36^{\circ} 15^{\prime} 13^{\prime \prime}$ & $116^{\circ} 31^{\prime} 31^{\prime \prime}$ & -- & 10.5 & -0.4 & 31.3 & 797.7 & $01-09-86$ & 361513116313101 \\
\hline 174 & $36^{\circ} 15^{\prime} 13^{\prime \prime}$ & $116^{\circ} 05^{\prime} 50^{\prime \prime}$ & 30 & -- & 0.15 & 14.8 & 774.2 & $02-17-76$ & 361513116055001 \\
\hline 175 & $36^{\circ} 15^{\prime} 12^{\prime \prime}$ & $116^{\circ} 05^{\prime} 32^{\prime \prime}$ & 9.8 & 3.17 & -- & 7.81 & 777.2 & $02-04-62$ & 361512116053201 \\
\hline 176 & $36^{\circ} 15^{\prime} 12^{\prime \prime}$ & $116^{\circ} 05^{\prime} 32^{\prime \prime}$ & 13 & -- & 0.28 & 11.7 & 773.3 & $02-17-76$ & 361512116053202 \\
\hline 178 & $36^{\circ} 14^{\prime} 55^{\prime \prime}$ & $116^{\circ} 03^{\prime} 49^{\prime \prime}$ & 37 & -- & - & 7.02 & 773.0 & $02-18-76$ & 361455116034901 \\
\hline 179 & $36^{\circ} 14^{\prime} 51^{\prime \prime}$ & $116^{\circ} 00^{\prime} 25^{\prime \prime}$ & 251 & 40.64 & - & 13.1 & 788.9 & $02-18-76$ & 351455116002001 \\
\hline 180 & $36^{\circ} 14^{\prime} 49^{\prime \prime}$ & $116^{\circ} 04^{\prime} 42^{\prime \prime}$ & - & -- & - & 7.61 & 772.4 & $02-18-76$ & 361449116044201 \\
\hline 182 & $36^{\circ} 14^{\prime} 46^{\prime \prime}$ & $116^{\circ} 00^{\prime} 56^{\prime \prime}$ & 207 & 30.48 & -- & 9.70 & 785.3 & $02-18-76$ & 361446116005601 \\
\hline 183 & $36^{\circ} 14^{\prime} 44^{\prime \prime}$ & $116^{\circ} 29^{\prime} 50^{\prime \prime}$ & -- & - & -- & 52 & 722.0 & $03-26-87$ & 361444116295001 \\
\hline 184 & $36^{\circ} 14^{\prime} 44^{\prime \prime}$ & $116^{\circ} 21^{\prime} 57^{\prime \prime}$ & 8.2 & 5.08 & -0.9 & 2.77 & 607.2 & $07-10-86$ & 361451116170800 \\
\hline 185 & $36^{\circ} 14^{\prime} 43^{\prime \prime}$ & $116^{\circ} 23^{\prime} 31^{\prime \prime}$ & 4.6 & 25.91 & -0.9 & 3.10 & 607.9 & $07-10-86$ & 361457116183400 \\
\hline 186 & $36^{\circ} 14^{\prime} 29^{\prime \prime}$ & $116^{\circ} 00^{\prime} 49^{\prime \prime}$ & 226 & 40.64 & -- & 13.1 & 780.9 & $02-18-76$ & 361429116004901 \\
\hline 187 & $36^{\circ} 14^{\prime} 29^{\prime \prime}$ & $116^{\circ} 00^{\prime} 18^{\prime \prime}$ & 234 & 40.64 & -- & 11.1 & 785.9 & $02-18-76$ & 361429116001801 \\
\hline 188 & $36^{\circ} 14^{\prime} 17^{\prime \prime}$ & $116^{\circ} 22^{\prime} 40^{\prime \prime}$ & 12 & 25.4 & -0.4 & 3.2 & 606.8 & $03-13-86$ & 361417116224001 \\
\hline 189 & $36^{\circ} 14^{\prime} 15^{\prime \prime}$ & $116^{\circ} 23^{\prime} 02^{\prime \prime}$ & 18 & 25.4 & -0.3 & 1.45 & 608.5 & $07-10-86$ & 361415116230200 \\
\hline 190 & $36^{\circ} 14^{\prime} 15^{\prime \prime}$ & $116^{\circ} 22^{\prime} 21^{\prime \prime}$ & 11 & 20.95 & -- & 1.87 & 608.1 & $07-10-86$ & 361406116173200 \\
\hline 191 & $36^{\circ} 14^{\prime} 15^{\prime \prime}$ & $116^{\circ} 22^{\prime} 21^{\prime \prime}$ & 8 & 20.32 & -0.7 & 2.3 & 607.7 & $06-14-85$ & 361415116222101 \\
\hline 192 & $36^{\circ} 14^{\prime} 15^{\prime \prime}$ & $116^{\circ} 22^{\prime} 21^{\prime \prime}$ & 5 & 15.24 & -0.1 & 1.4 & 608.6 & $06-14-85$ & 361415116222102 \\
\hline
\end{tabular}


Table 2. Ground-water levels and well data of wells located in the Death Valley Junction, California-Nevada, 30-minute $\times 60$-minute quadrangle--Continued

\begin{tabular}{|c|c|c|c|c|c|c|c|c|c|}
\hline $\begin{array}{c}\text { Site } \\
\text { number } \\
\text { (pl. 2) }\end{array}$ & Latitude & Longitude & $\begin{array}{l}\text { Depth } \\
\text { of well } \\
\text { below } \\
\text { land } \\
\text { surface } \\
\text { (m) }\end{array}$ & $\begin{array}{l}\text { Diameter of } \\
\text { casing } \\
\text { (cm) }\end{array}$ & $\begin{array}{l}\text { Height of } \\
\text { measuring } \\
\text { point above } \\
\text { land surface } \\
\text { (m) }\end{array}$ & $\begin{array}{l}\text { Water } \\
\text { level } \\
\text { below } \\
\text { land } \\
\text { surface } \\
\text { (m) }\end{array}$ & $\begin{array}{l}\text { Water } \\
\text { level } \\
\text { altitude } \\
\text { (m) }\end{array}$ & $\begin{array}{l}\text { Date of } \\
\text { measurement }\end{array}$ & NWIS Slte-ID \\
\hline 195 & $36^{\circ} 14^{\prime} 13^{\prime \prime}$ & $116^{\circ} 22^{\prime} 12^{\prime \prime}$ & 6 & 15.24 & -1.0 & 3.2 & 605.8 & $06-14-85$ & 361413116221201 \\
\hline 196 & $36^{\circ} 14^{\prime} 13^{\prime \prime}$ & $116^{\circ} 22^{\prime} 12^{\prime \prime}$ & 6 & -- & -0.6 & 3.2 & 606.8 & $03-18-86$ & 361413116221202 \\
\hline 197 & $36^{\circ} 14^{\prime} 13^{\prime \prime}$ & $116^{\circ} 22^{\prime} 12^{\prime \prime}$ & 9 & 15.24 & -0.2 & 2.9 & 607.1 & $06-14-85$ & 361413116221203 \\
\hline 198 & $36^{\circ} 14^{\prime} 12^{\prime \prime}$ & $116^{\circ} 22^{\prime} 30^{\prime \prime}$ & 12 & 25.91 & -0.6 & 2.27 & 605.7 & $07-10-86$ & 361406116173900 \\
\hline 199 & $36^{\circ} 14^{\prime} 11^{\prime \prime}$ & $116^{\circ} 10^{\prime} 03^{\prime \prime}$ & 8.2 & 3.81 & -- & 5.35 & 743.6 & $01-28-76$ & 361411116100301 \\
\hline 200 & $36^{\circ} 14^{\prime} 09^{\prime \prime}$ & $116^{\circ} 06^{\prime} 39^{\prime \prime}$ & 202 & 41.14 & -- & Flows & Flows & $02-04-62$ & 361415116002001 \\
\hline 201 & $36^{\circ} 14^{\prime} 08^{\prime \prime}$ & $116^{\circ} 09^{\prime} 18^{\prime \prime}$ & 52 & -. & -- & 2.63 & 747.4 & $01-28-76$ & 361927116094301 \\
\hline 202 & $36^{\circ} 14^{\prime} 06^{\prime \prime}$ & $116^{\circ} 11^{\prime} 06^{\prime \prime}$ & 13 & 3.05 & -- & 7.11 & 741.9 & $01-28-76$ & 361406116110601 \\
\hline 203 & $36^{\circ} 14^{\prime} 05^{\prime \prime}$ & $116^{\circ} 03^{\prime} 32^{\prime \prime}$ & 61 & 35.56 & -0.5 & 10.8 & 769.2 & $03-27-91$ & 361405116033201 \\
\hline 204 & $36^{\circ} 14^{\prime} 01^{\prime \prime}$ & $116^{\circ} 04^{\prime} 21^{\prime \prime}$ & 61 & 25.40 & -- & 5.03 & 773.0 & $02-03-62$ & 361401116042101 \\
\hline 205 & $36^{\circ} 13^{\prime} 55^{\prime \prime}$ & $116^{\circ} 09^{\prime} 04^{\prime \prime}$ & 37 & -- & -- & 3.76 & 747.2 & $01-28-76$ & 361903116093001 \\
\hline 206 & $36^{\circ} 13^{\prime} 52^{\prime \prime}$ & $116^{\circ} 00^{\prime} 53^{\prime \prime}$ & - & -- & - & 12.5 & 779.5 & $02-18-76$ & 361352116005301 \\
\hline 207 & $36^{\circ} 13^{\prime} 34^{\prime \prime}$ & $116^{\circ} 01^{\prime} 56^{\prime \prime}$ & 119 & 40.64 & -- & 9.10 & 776.9 & $02-18-76$ & 361334116015601 \\
\hline 209 & $36^{\circ} 13^{\prime} 33^{\prime \prime}$ & $116^{\circ} 05^{\prime} 09^{\prime \prime}$ & 60 & 24.13 & $\cdots$ & 6.47 & 768.5 & $02-03-62$ & 361333116050901 \\
\hline 210 & $36^{\circ} 13^{\prime} 31^{\prime \prime}$ & $116^{\circ} 00^{\prime} 36^{\prime \prime}$ & -- & -- & -- & 11.5 & 781.5 & $02-18-76$ & 361331116003601 \\
\hline 211 & $36^{\circ} 13^{\prime} 31^{\prime \prime}$ & $116^{\circ} 00^{\prime} 18^{\prime \prime}$ & 305 & 45.72 & -- & 7.74 & 788.3 & $02-04-62$ & 361333116001801 \\
\hline 212 & $36^{\circ} 13^{\prime} 27^{\prime \prime}$ & $116^{\circ} 22^{\prime} 49^{\prime \prime}$ & 4.5 & 5.08 & - & 3.88 & 605.1 & $07-10-86$ & 361327116224900 \\
\hline 213 & $36^{\circ} 13^{\prime} 27^{\prime \prime}$ & $116^{\circ} 00^{\prime} 18^{\prime \prime}$ & 198 & 35.56 & -- & -8.02 & 804.0 & $02-04-62$ & 361327116001801 \\
\hline 215 & $36^{\circ} 13^{\prime} 21^{\prime \prime}$ & $116^{\circ} 04^{\prime} 18^{\prime \prime}$ & -- & -. & -- & 7.47 & 769.5 & $02-18-76$ & 361321116041801 \\
\hline 216 & $36^{\circ} 13^{\prime} 13^{\prime \prime}$ & $116^{\circ} 06^{\prime} 12^{\prime \prime}$ & 23 & -. & -- & 13.2 & 763.8 & $02-20-76$ & 361313116061201 \\
\hline 217 & $36^{\circ} 13^{\prime} 11^{\prime \prime}$ & $116^{\circ} 02^{\prime} 55^{\prime \prime}$ & -- & -. & -- & 8.90 & 772.1 & $02-18-76$ & 361311116025501 \\
\hline 218 & $36^{\circ} 13^{\prime} 08^{\prime \prime}$ & $116^{\circ} 01^{\prime} 24^{\prime \prime}$ & 122 & 30.48 &.- & 7.78 & 781.2 & $02-27-75$ & 361308116012401 \\
\hline 219 & $36^{\circ} 13^{\prime} 08^{\prime \prime}$ & $116^{\circ} 01^{\prime} 05^{\prime \prime}$ & 122 & 30.48 & $\ldots$ & 5.50 & 783.5 & $02-18-76$ & 361308116010501 \\
\hline
\end{tabular}




\begin{tabular}{|c|c|c|c|c|c|c|c|c|c|}
\hline $\begin{array}{c}\text { Site } \\
\text { number } \\
\text { (pl. 2) }\end{array}$ & Latitude & Longitude & $\begin{array}{l}\text { Depth } \\
\text { of well } \\
\text { below } \\
\text { land } \\
\text { surface } \\
\text { (m) }\end{array}$ & $\begin{array}{l}\text { Diameter of } \\
\text { casing } \\
\text { (cm) }\end{array}$ & $\begin{array}{l}\text { Height of } \\
\text { measuring } \\
\text { point above } \\
\text { land surface } \\
\text { (m) }\end{array}$ & $\begin{array}{l}\text { Water } \\
\text { level } \\
\text { below } \\
\text { land } \\
\text { surface } \\
\text { (m) }\end{array}$ & $\begin{array}{l}\text { Water } \\
\text { level } \\
\text { altitude } \\
(\mathbf{m})\end{array}$ & $\begin{array}{c}\text { Date of } \\
\text { measurement }\end{array}$ & NWIS Site-ID \\
\hline 220 & $36^{\circ} 13^{\prime} 08^{\prime \prime}$ & $116^{\circ} 00^{\prime} 52^{\prime \prime}$ & 129 & 35.56 & - & 7.78 & 783.2 & $02-18-76$ & 361308116005201 \\
\hline 221 & $36^{\circ} 12^{\prime} 48^{\prime \prime}$ & $116^{\circ} 11^{\prime} 38^{\prime \prime}$ & 10 & 3.30 & - & 7.08 & 741.9 & $01-28-76$ & 361248116113801 \\
\hline 222 & $36^{\circ} 12^{\prime} 46^{\prime \prime}$ & $116^{\circ} 03^{\prime} 31^{\prime \prime}$ & 76 & 30.48 & -. & 8.78 & 769.2 & $02-18-76$ & 361246116033101 \\
\hline 223 & $36^{\circ} 12^{\prime} 46^{\prime \prime}$ & $116^{\circ} 03^{\prime} 00^{\prime \prime}$ & 244 & 35.56 & -- & 11.6 & 770.4 & $06-05-74$ & 361246116030001 \\
\hline 224 & $36^{\circ} 12^{\prime} 42^{\prime \prime}$ & $116^{\circ} 00^{\prime} 42^{\prime \prime}$ & -- & -. & -- & 8.56 & 782.4 & $02-28-75$ & 361242116004201 \\
\hline 226 & $36^{\circ} 12^{\prime} 31^{\prime \prime}$ & $116^{\circ} 02^{\prime} 29^{\prime \prime}$ & 41 & 76.20 & -- & 7.09 & 775.9 & $02-04-62$ & 361231116022901 \\
\hline 228 & $36^{\circ} 12^{\prime} 22^{\prime \prime}$ & $116^{\circ} 03^{\prime} 00^{\prime \prime}$ & 67 & 35.56 & -- & 8.74 & 771.3 & $02-18-76$ & 361222116030001 \\
\hline 229 & $36^{\circ} 12^{\prime} 22^{\prime \prime}$ & $116^{\circ} 00^{\prime} 44^{\prime \prime}$ & -- & -- & -- & 8.07 & 782.9 & $02-18-76$ & 361222116004401 \\
\hline 230 & $36^{\circ} 12^{\prime} 18^{\prime \prime}$ & $116^{\circ} 09^{\prime} 19^{\prime \prime}$ & 9.1 & 3.05 & -- & 3.31 & 746.7 & $03-30-60$ & 361218116091901 \\
\hline 231 & $36^{\circ} 12^{\prime} 18^{\prime \prime}$ & $116^{\circ} 00^{\prime} 51^{\prime \prime}$ & 160 & 35.56 & - & 5.00 & 785.0 & $02-18-76$ & 361218116005101 \\
\hline 232 & $36^{\circ} 12^{\prime} 17^{\prime \prime}$ & $116^{\circ} 02^{\prime} 10^{\prime \prime}$ & 275 & 35.56 & -- & 6.73 & 775.3 & 02-04-62 & 361219116021001 \\
\hline 233 & $36^{\circ} 12^{\prime} 17^{\prime \prime}$ & $116^{\circ} 01^{\prime} 00^{\prime \prime}$ & 107 & 35.51 & -- & 7.49 & 790.5 & $02-18-76$ & 361217116010001 \\
\hline 234 & $36^{\circ} 12^{\prime} 16^{\prime \prime}$ & $116^{\circ} 06^{\prime} 09^{\prime \prime}$ & 152 & 35.56 & -. & 16.1 & 754.9 & $02-18-76$ & 361216116060901 \\
\hline 235 & $36^{\circ} 12^{\prime} 16^{\prime \prime}$ & $116^{\circ} 05^{\prime} 37^{\prime \prime}$ & 168 & 35.56 & -- & 11.7 & 762.3 & $02-18-76$ & 361216116053701 \\
\hline 236 & $36^{\circ} 12^{\prime} 13^{\prime \prime}$ & $116^{\circ} 01^{\prime} 55^{\prime \prime}$ & 5.5 & -. & -- & 5.00 & 781.0 & $03-23-54$ & 361213116015501 \\
\hline 237 & $36^{\circ} 12^{\prime} 13^{\prime \prime}$ & $116^{\circ} 01^{\prime} 55^{\prime \prime}$ & 21 & -- & -. & 7.11 & 778.9 & $02-04-62$ & 361213116015502 \\
\hline 238 & $36^{\circ} 12^{\prime} 10^{\prime \prime}$ & $116^{\circ} 22^{\prime} 57^{\prime \prime}$ & -- & 5.08 & -0.5 & 24.0 & 607.0 & $01-14-87$ & 361210116225701 \\
\hline 239 & $36^{\circ} 12^{\prime} 10^{\prime \prime}$ & $116^{\circ} 22^{\prime} 57^{\prime \prime}$ & 40 & 3.17 & -0.5 & 23.4 & 607.6 & $01-14-87$ & 361210116225702 \\
\hline 240 & $36^{\circ} 12^{\prime} 09^{\prime \prime}$ & $116^{\circ} 06^{\prime} 14^{\prime \prime}$ & 91 & 35.56 & -- & 20.6 & 759.4 & 03-27-91 & 361209116061401 \\
\hline 241 & $36^{\circ} 12^{\prime} 04^{\prime \prime}$ & $116^{\circ} 06^{\prime} 03^{\prime \prime}$ & 152 & 35.56 & 0.2 & 15.3 & 755.7 & $03-27-91$ & 361204116060301 \\
\hline 242 & $36^{\circ} 11^{\prime} 58^{\prime \prime}$ & $116^{\circ} 24^{\prime} 05^{\prime \prime}$ & 427 & 5.08 & -0.8 & 89.6 & 584.4 & $01-14-87$ & 361158116240501 \\
\hline 243 & $36^{\circ} 11^{\prime} 58^{\prime \prime}$ & $116^{\circ} 24^{\prime} 05^{\prime \prime}$ & 107 & 3.17 & -0.8 & 94.0 & 580.0 & $01-14-87$ & 361158116240502 \\
\hline 245 & $36^{\circ} 11^{\prime} 49^{\prime \prime}$ & $116^{\circ} 00^{\prime} 21^{\prime \prime}$ & 107 & 40.64 & -- & 2.97 & 796.0 & $02-19-76$ & 361149116002101 \\
\hline 246 & $36^{\circ} 11^{\prime} 46^{\prime \prime}$ & $116^{\circ} 04^{\prime} 28^{\prime \prime}$ & -. & -- & -- & 8.43 & 766.6 & $02-18-76$ & 361146116042801 \\
\hline
\end{tabular}


Table 2. Ground-water levels and well data of wells located in the Death Valley Junction, California-Nevada, 30-minute $\times 60-$ minute quadrangle--Continued

\begin{tabular}{|c|c|c|c|c|c|c|c|c|c|}
\hline $\begin{array}{c}\text { Slte } \\
\text { number } \\
\text { (pl. 2) }\end{array}$ & Latitude & Longitude & $\begin{array}{l}\text { Depth } \\
\text { of well } \\
\text { below } \\
\text { land } \\
\text { surface } \\
\text { (m) }\end{array}$ & $\begin{array}{l}\text { Diameter of } \\
\text { casing } \\
\text { (cm) }\end{array}$ & $\begin{array}{l}\text { Height of } \\
\text { measuring } \\
\text { point above } \\
\text { land surface } \\
\text { (m) }\end{array}$ & $\begin{array}{l}\text { Water } \\
\text { level } \\
\text { below } \\
\text { land } \\
\text { surface } \\
\text { (m) }\end{array}$ & $\begin{array}{l}\text { Water } \\
\text { level } \\
\text { altitude } \\
\text { (m) }\end{array}$ & $\begin{array}{l}\text { Date of } \\
\text { measurement }\end{array}$ & NWIS Site-1D \\
\hline 247 & $36^{\circ} 11^{\prime} 18^{\prime \prime}$ & $116^{\circ} 00^{\prime} 43^{\prime \prime}$ & 107 & 30.48 & -- & 1.73 & 790.3 & $02-19-76$ & 361118116004301 \\
\hline 248 & $36^{\circ} 10^{\prime} 34^{\prime \prime}$ & $116^{\circ} 04^{\prime} 59^{\prime \prime}$ & 61 & 33.02 & -- & 9.56 & 758.4 & $02-18-76$ & 361034116045901 \\
\hline 249 & $36^{\circ} 10^{\prime} 31^{\prime \prime}$ & $116^{\circ} 05^{\prime} 16^{\prime \prime}$ & 16 & -- & -- & 7.05 & 759.9 & $02-07-62$ & 361031116051601 \\
\hline 250 & $36^{\circ} 10^{\prime} 30^{\prime \prime}$ & $116^{\circ} 05^{\prime} 17^{\prime \prime}$ & - & -- & -- & 9.10 & 757.9 & $02-20-76$ & 361030116051701 \\
\hline 251 & $36^{\circ} 10^{\prime} 30^{\prime \prime}$ & $116^{\circ} 01^{\prime} 43^{\prime \prime}$ & 9.1 & - & -- & 6.07 & 777.9 & $02-19-76$ & 361030116014301 \\
\hline 252 & $36^{\circ} 10^{\prime} 29^{\prime \prime}$ & $116^{\circ} 01^{\prime} 41^{\prime \prime}$ & 60 & 25.40 & -. & 6.39 & 777.6 & $02-03-62$ & 361029116014101 \\
\hline 253 & $36^{\circ} 10^{\prime} 14^{\prime \prime}$ & $116^{\circ} 04^{\prime} 42^{\prime \prime}$ & 77 & 30.48 & -- & 10.7 & 758.3 & $02-18-76$ & 361014116044201 \\
\hline 254 & $36^{\circ} 10^{\prime} 12^{\prime \prime}$ & $116^{\circ} 04^{\prime} 47^{\prime \prime}$ & 77 & 30.48 & 0.0 & 13.6 & 754.4 & $03-27-91$ & 361012116044701 \\
\hline 255 & $36^{\circ} 09^{\prime} 51^{\prime \prime}$ & $116^{\circ} 07^{\prime} 22^{\prime \prime}$ & 14 & 3.30 & -- & 10.7 & 744.3 & $04-17-63$ & 360951116072201 \\
\hline 257 & $36^{\circ} 09^{\prime} 38^{\prime \prime}$ & $115^{\circ} 59^{\prime} 35^{\prime \prime}$ & 153 & 35.56 & -- & 7.45 & 791.5 & $02-18-76$ & 360938115593501 \\
\hline 258 & $36^{\circ} 09^{\prime} 30^{\prime \prime}$ & $116^{\circ} 05^{\prime} 20^{\prime \prime}$ & 6.7 & -- & -. & 5.71 & 755.3 & $02-03-62$ & 360930116052001 \\
\hline 259 & $36^{\circ} 09^{\prime} 30^{\prime \prime}$ & $116^{\circ} 05^{\prime} 20^{\prime \prime}$ & 13 & 3.81 & -- & 7.13 & 753.9 & 03-07-77 & 360930116052002 \\
\hline 260 & $36^{\circ} 08^{\prime} 45^{\prime \prime}$ & $116^{\circ} 02^{\prime} 25^{\prime \prime}$ & 17 & 31.75 & -- & 13.1 & 760.9 & $02-03-62$ & 360845116022501 \\
\hline 261 & $36^{\circ} 08^{\prime} 45^{\prime \prime}$ & $116^{\circ} 02^{\prime} 25^{\prime \prime}$ & 19 & -- & -- & 15.8 & 758.2 & $03-07-77$ & 360845116022502 \\
\hline 262 & $36^{\circ} 08^{\prime} 45^{\prime \prime}$ & $115^{\circ} 59^{\prime} 36^{\prime \prime}$ & 128 & 40.64 & -- & 10.4 & 787.6 & $03-19-76$ & 360845115593601 \\
\hline 263 & $36^{\circ} 07^{\prime} 56^{\prime \prime}$ & $116^{\circ} 00^{\prime} 35^{\prime \prime}$ & 19 & -- & -- & 13.2 & 773.8 & $03-07-77$ & 360756116003501 \\
\hline 264 & $36^{\circ} 07^{\prime} 51^{\prime \prime}$ & $116^{\circ} 05^{\prime} 59^{\prime \prime}$ & 31 & 3.30 & -- & 18.1 & 739.9 & $02-05-62$ & 360751116055901 \\
\hline 265 & $36^{\circ} 07^{\prime} 48^{\prime \prime}$ & $116^{\circ} 05^{\prime} 23^{\prime \prime}$ & -- & -- & -- & 18.4 & 740.6 & $03-07-77$ & 360748116052301 \\
\hline 266 & $36^{\circ} 07^{\prime} 02^{\prime \prime}$ & $116^{\circ} 03^{\prime} 35^{\prime \prime}$ & 26 & 3.81 & -- & 16.3 & 748.7 & $02-20-76$ & 360702116033501 \\
\hline 267 & $36^{\circ} 04^{\prime} 45^{\prime \prime}$ & $116^{\circ} 01^{\prime} 54^{\prime \prime}$ & 23 & 3.30 & -- & 17.3 & 747.7 & $01-23-76$ & 360445116015401 \\
\hline 268 & $36^{\circ} 02^{\prime} 32^{\prime \prime}$ & $116^{\circ} 00^{\prime} 36^{\prime \prime}$ & 61 & 3.81 & -- & 35.6 & 729.4 & $11-01-77$ & 360232116003601 \\
\hline
\end{tabular}


The following number is for U.S. Department of Energy Office of Civilian Radioactive Waste Management Records Management purposes only and should not be used when ordering this publication: Accession number - MOL.19940908.0078. The distribution code for this report is UC-814. 


\section{U.S. DEPARTMENT OF THE INTERIOR U.S. GEOLOGICAL SURVEY}

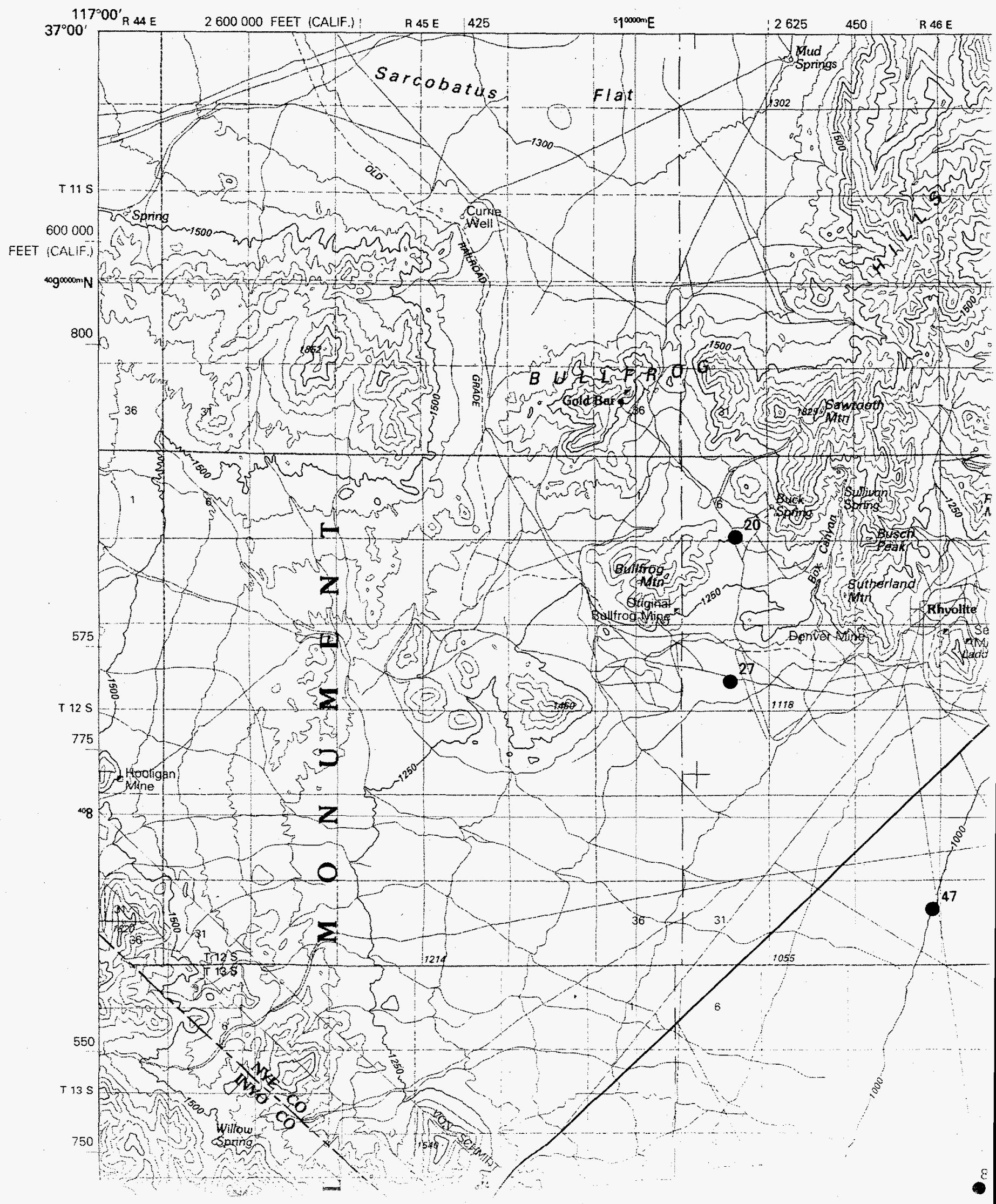




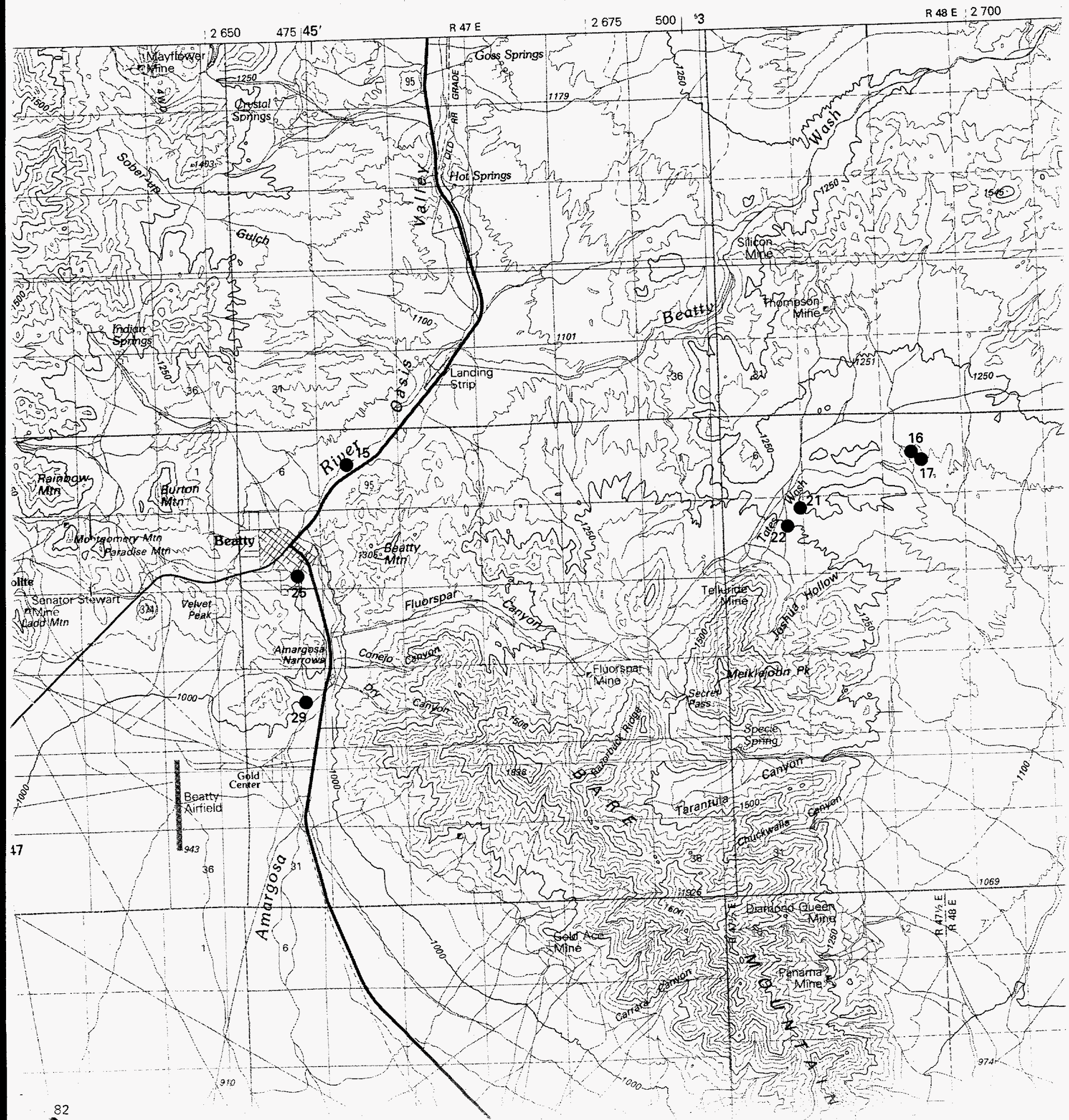




\section{Prepared in cooperation with the U.S. DEPARTMENT OF ENERGY NEVADA OPERATIONS OFFICE}
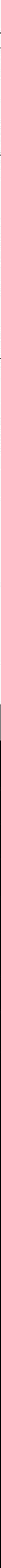


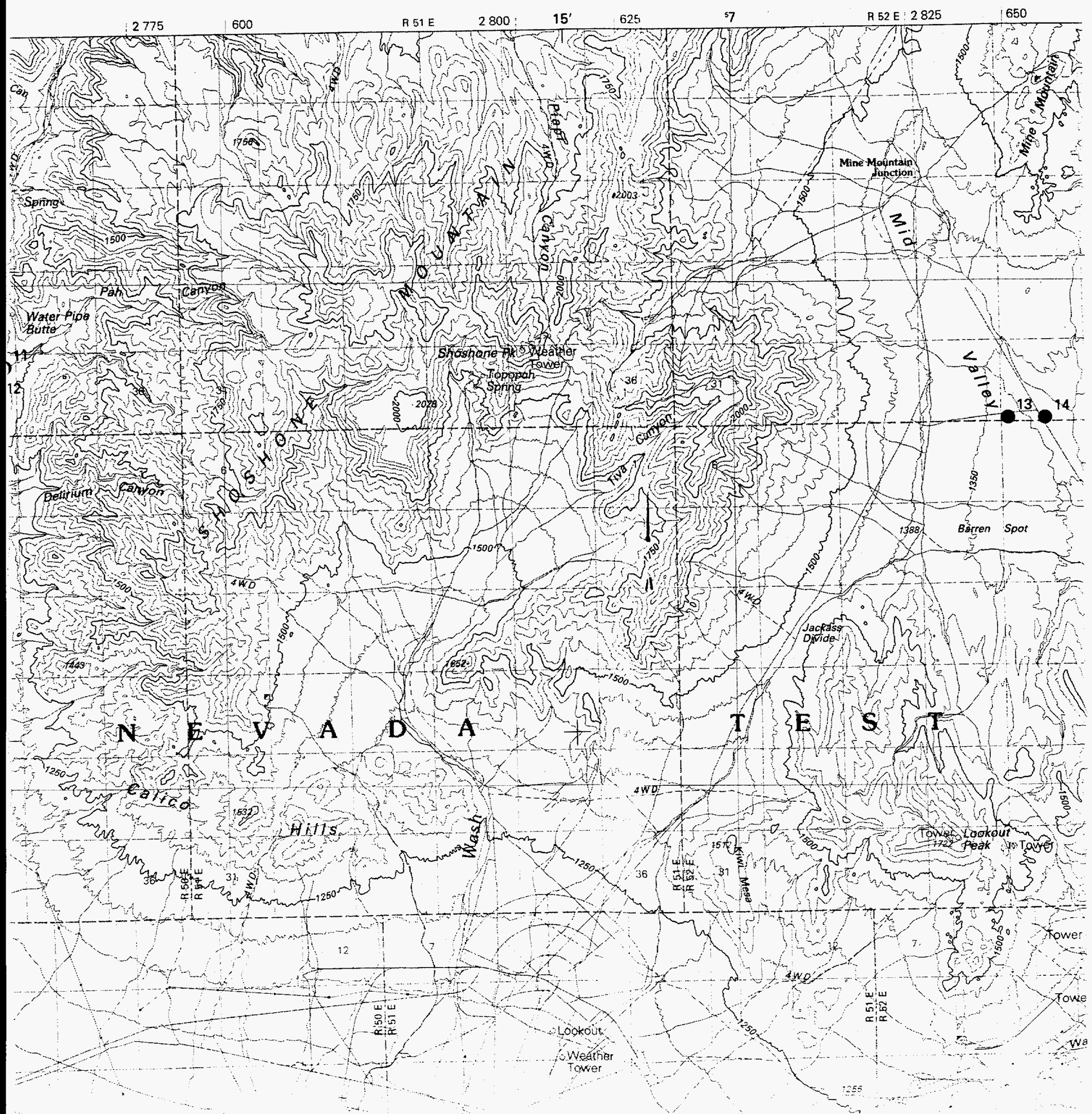




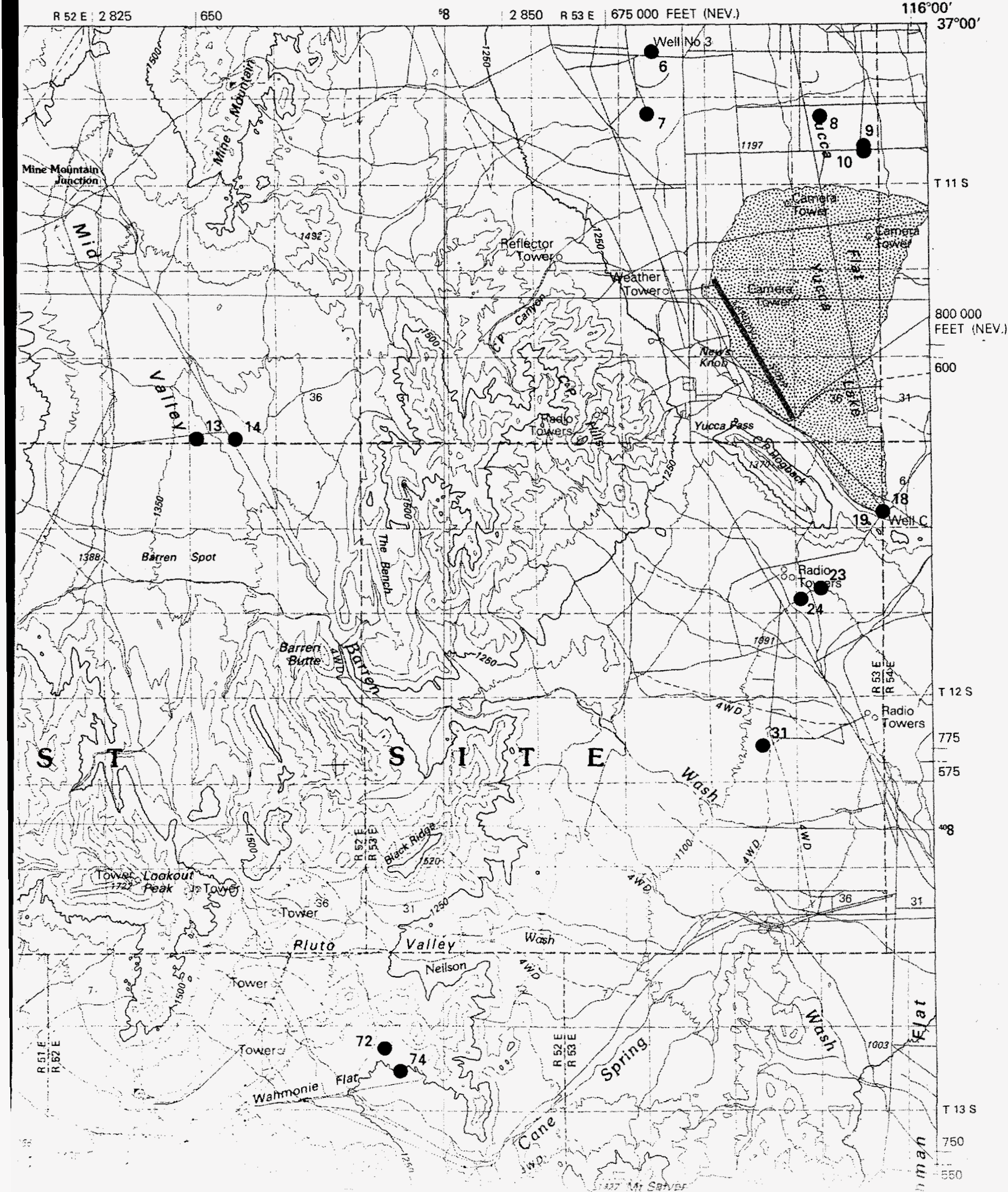




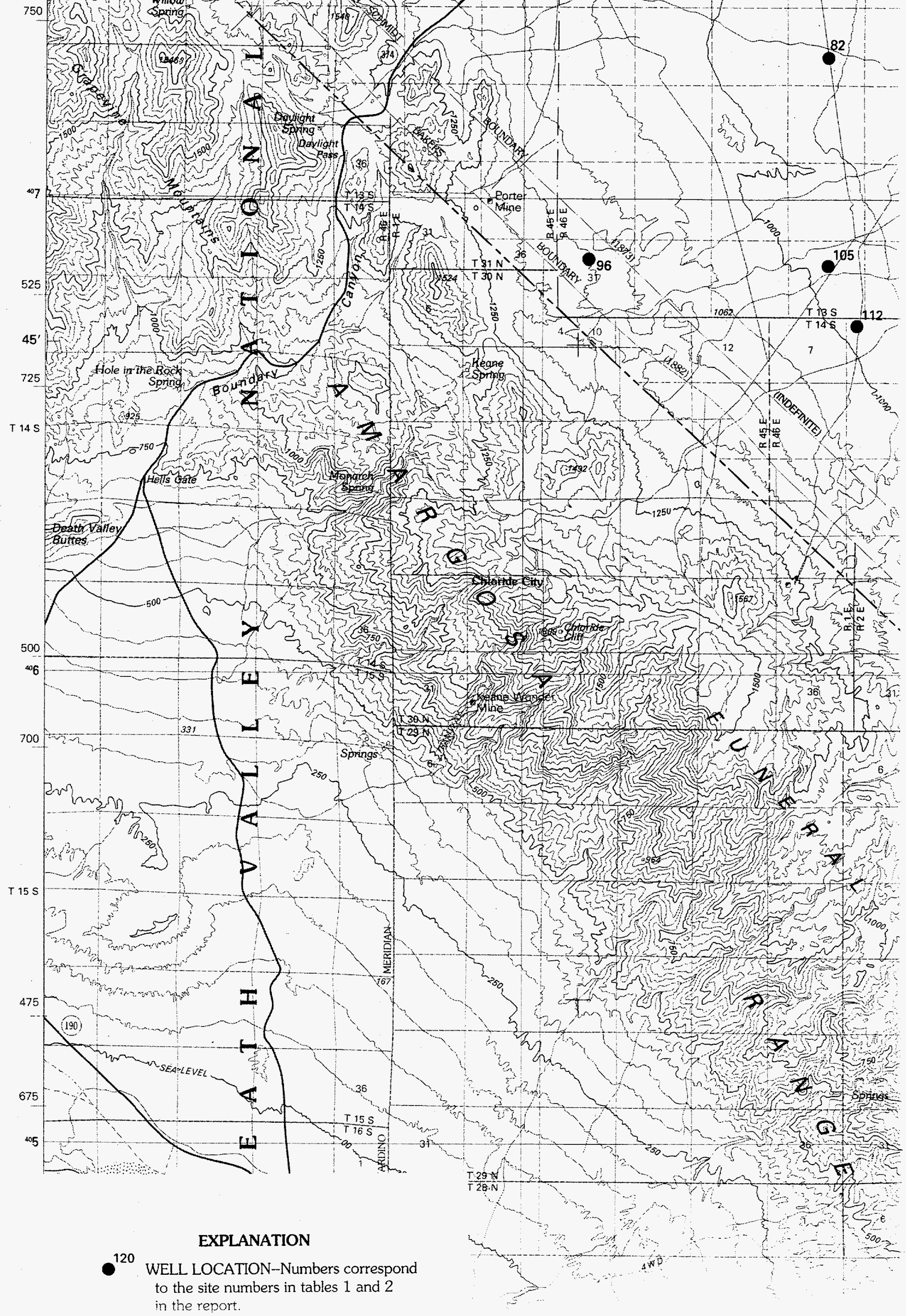




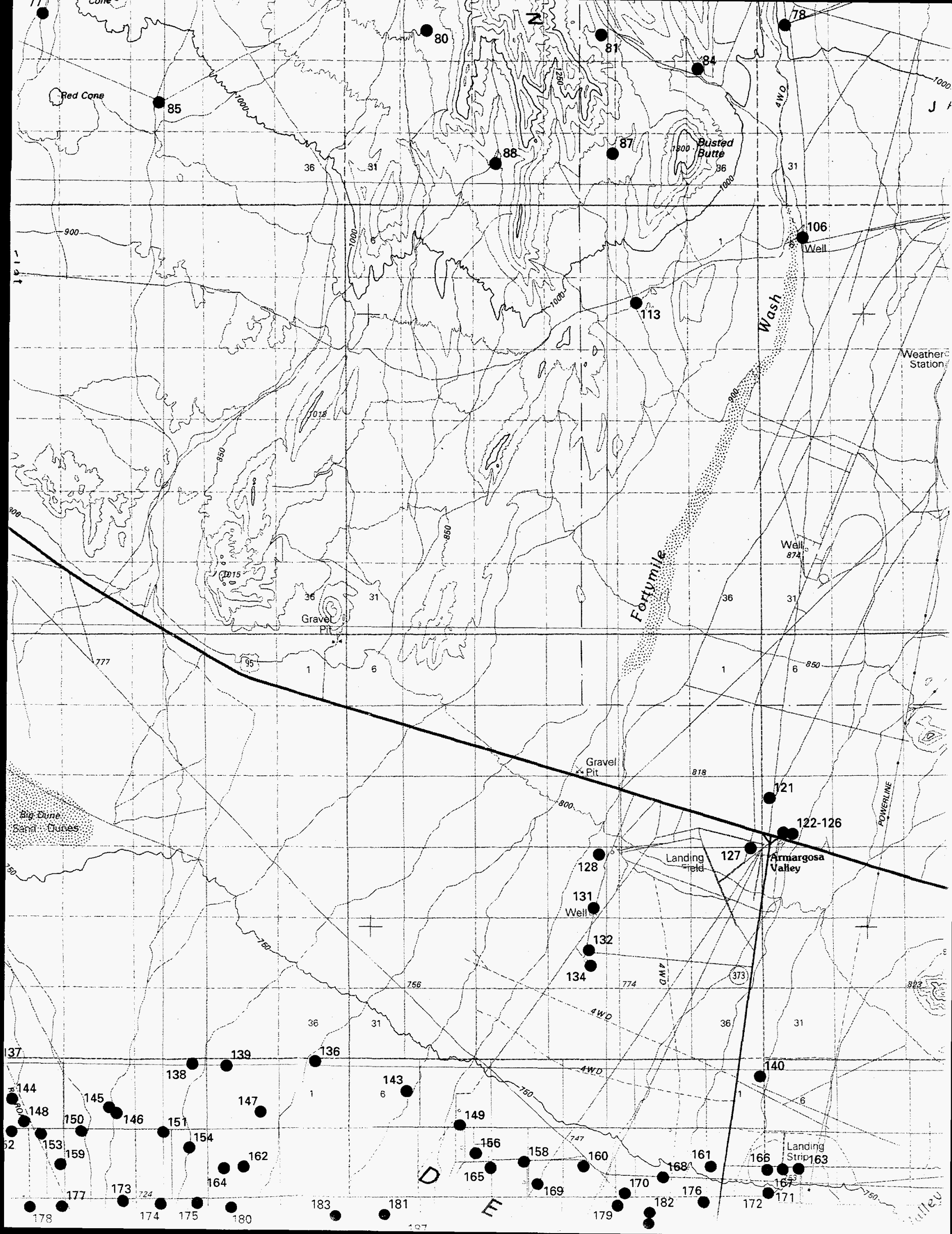



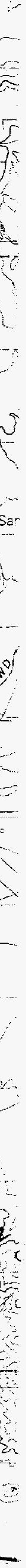


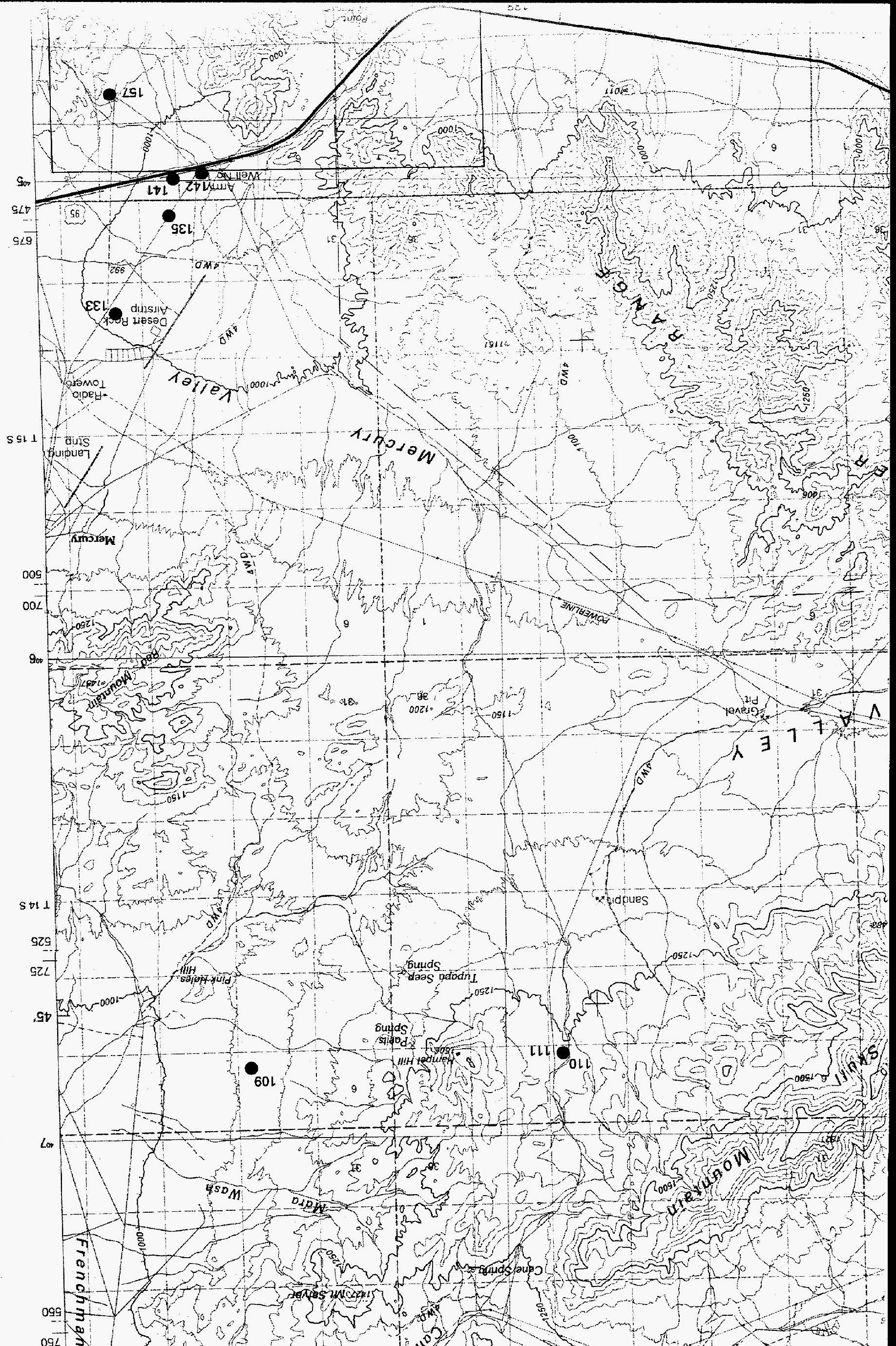




\section{EXPLANATION}

120 WELL LOCATION--Numbers correspond to the site numbers in tables 1 and 2 in the report.

T16 S

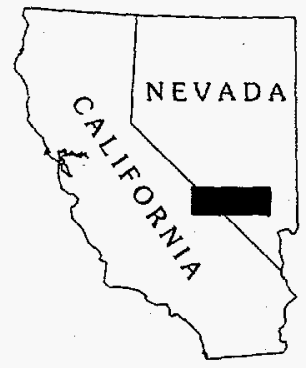

MAP LOCATION

T 16/2s

$36^{\circ} 30^{\prime}$

$117^{\circ} 00^{\prime}$

R $46 \mathrm{E}$

R $47 \mathrm{E}$

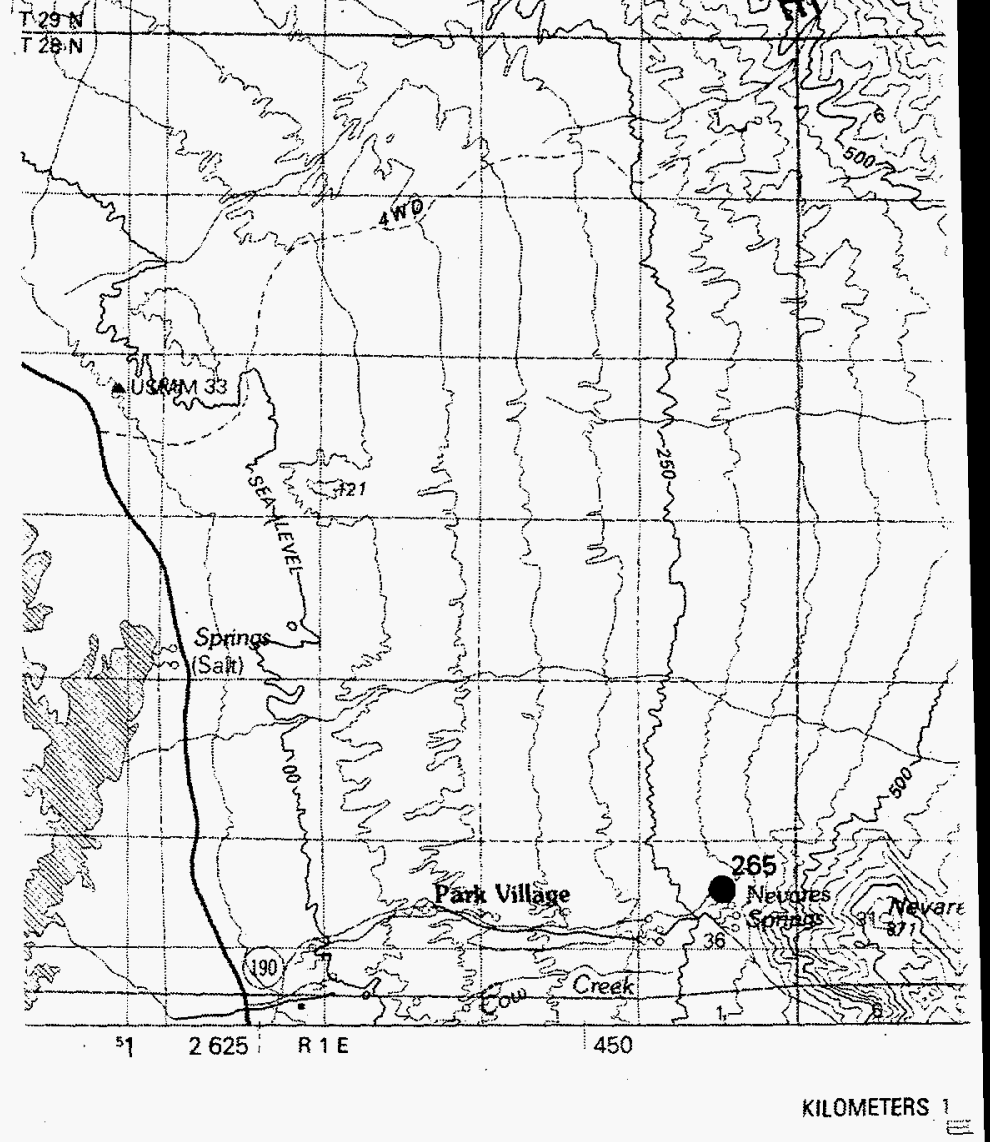

1:100,000, Beatty, Nevada-California, 1986
KILOMETERS

MILES 1 


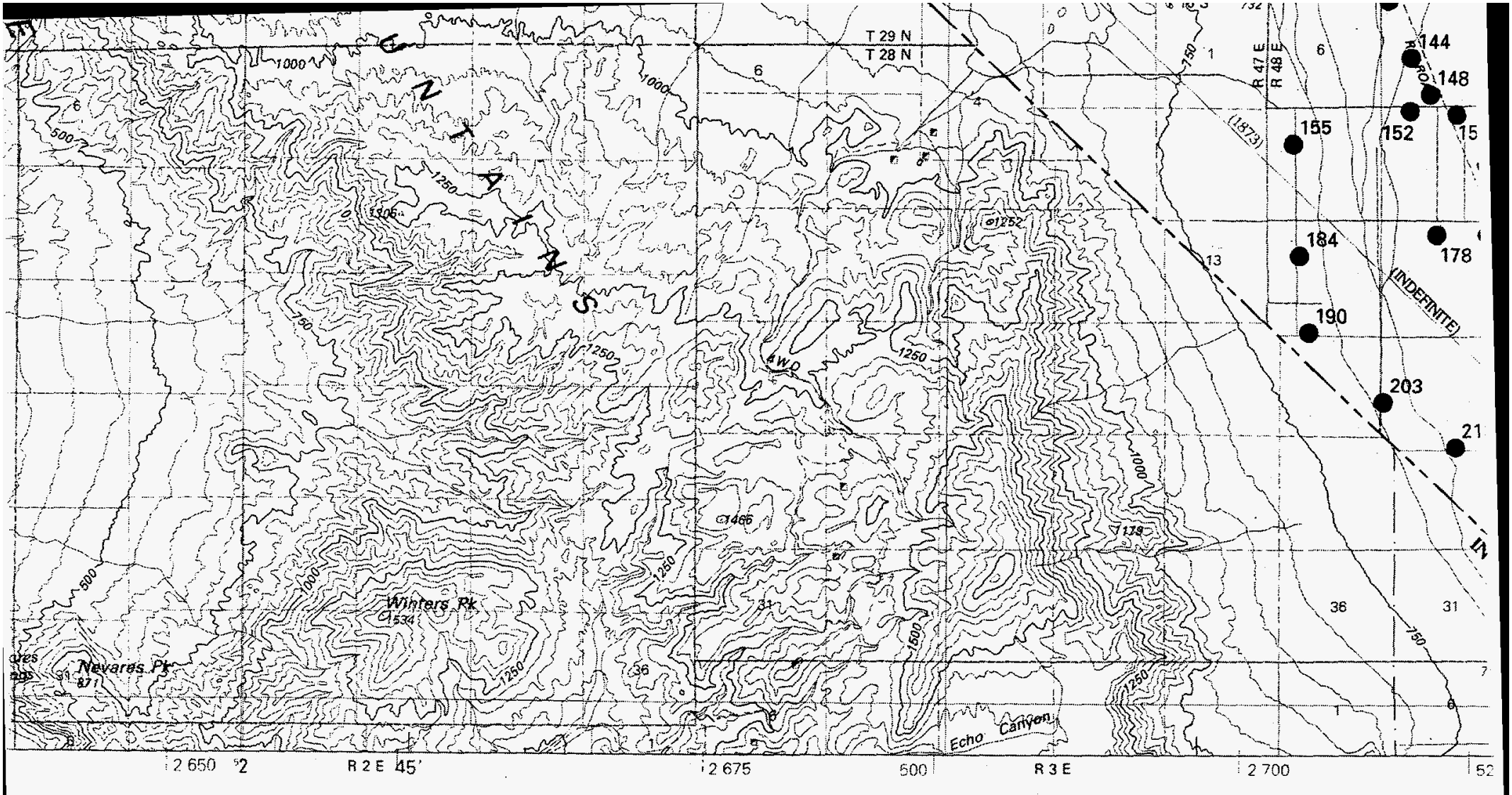

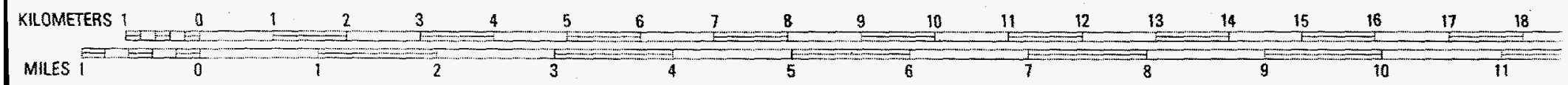




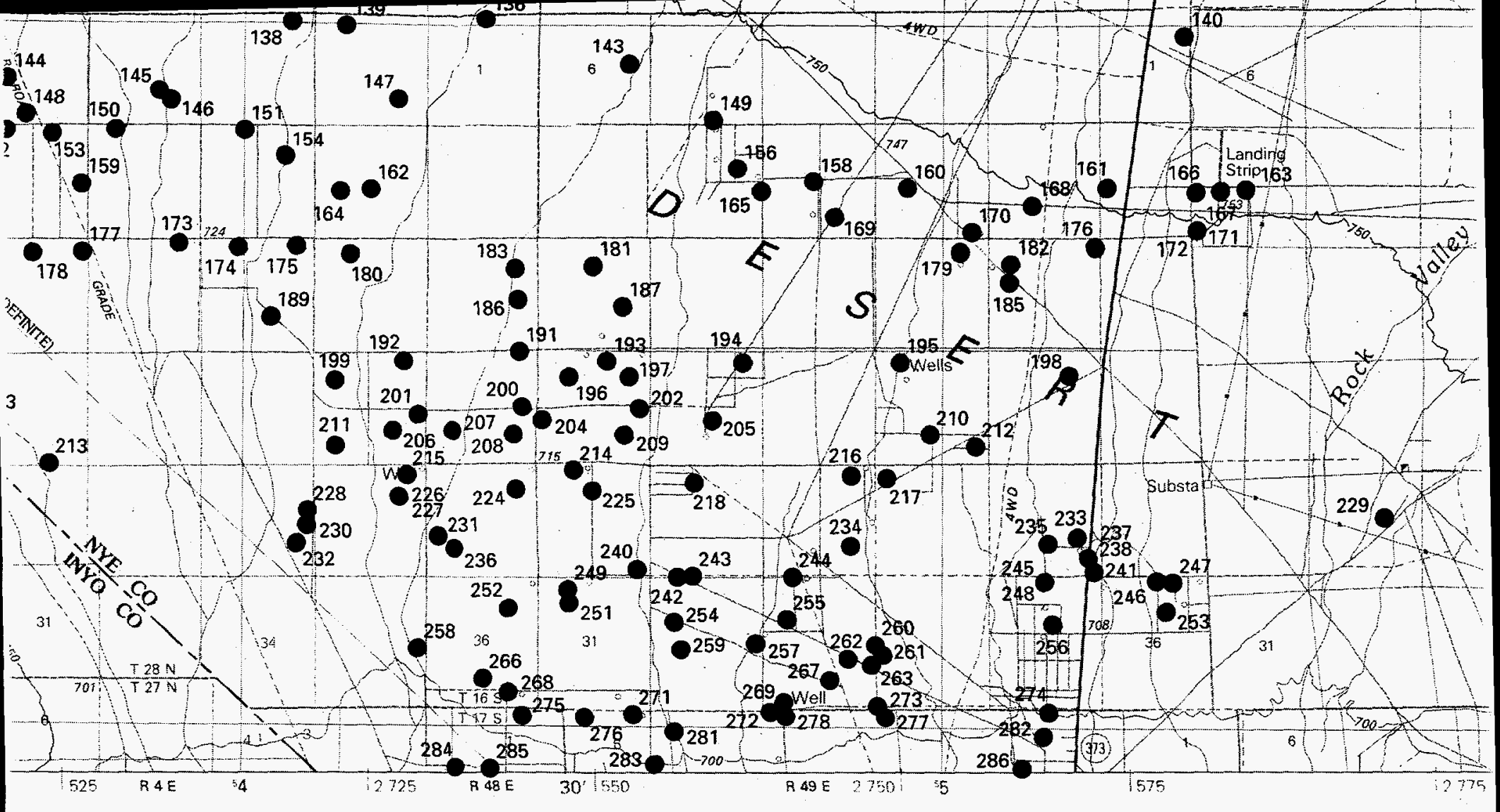

SCALE $1: 100,000$
11

CONTOUR INTERVAL 50 METERS

1000
$5=15$

5000

NATIONAL GEODETIC VERTICAL DATUM OF 1929

OF DATA-BASE WELLS IN THE BEATTY, NEVADA-1 


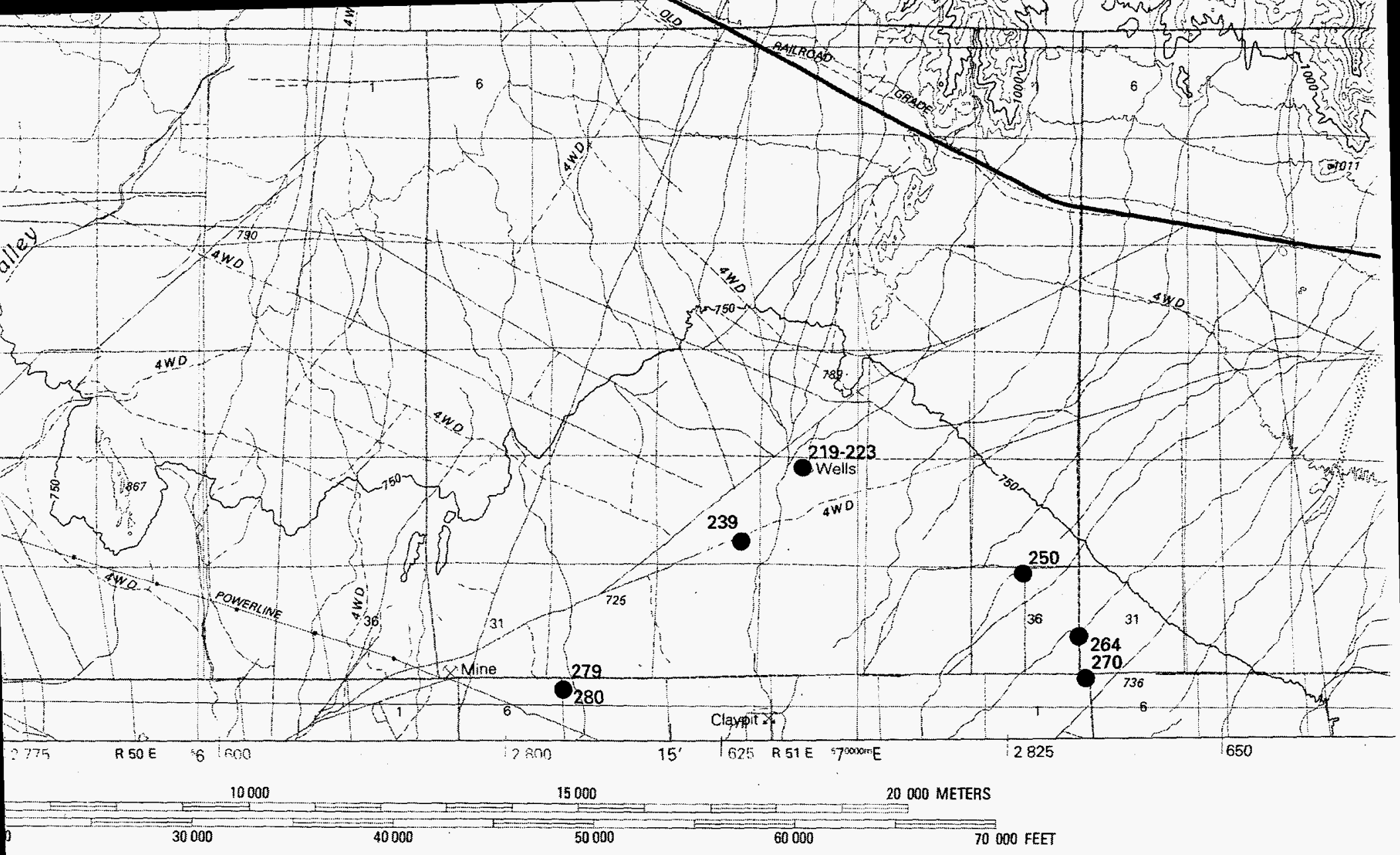

\section{A-CALIFORNIA QUADRANGLE}




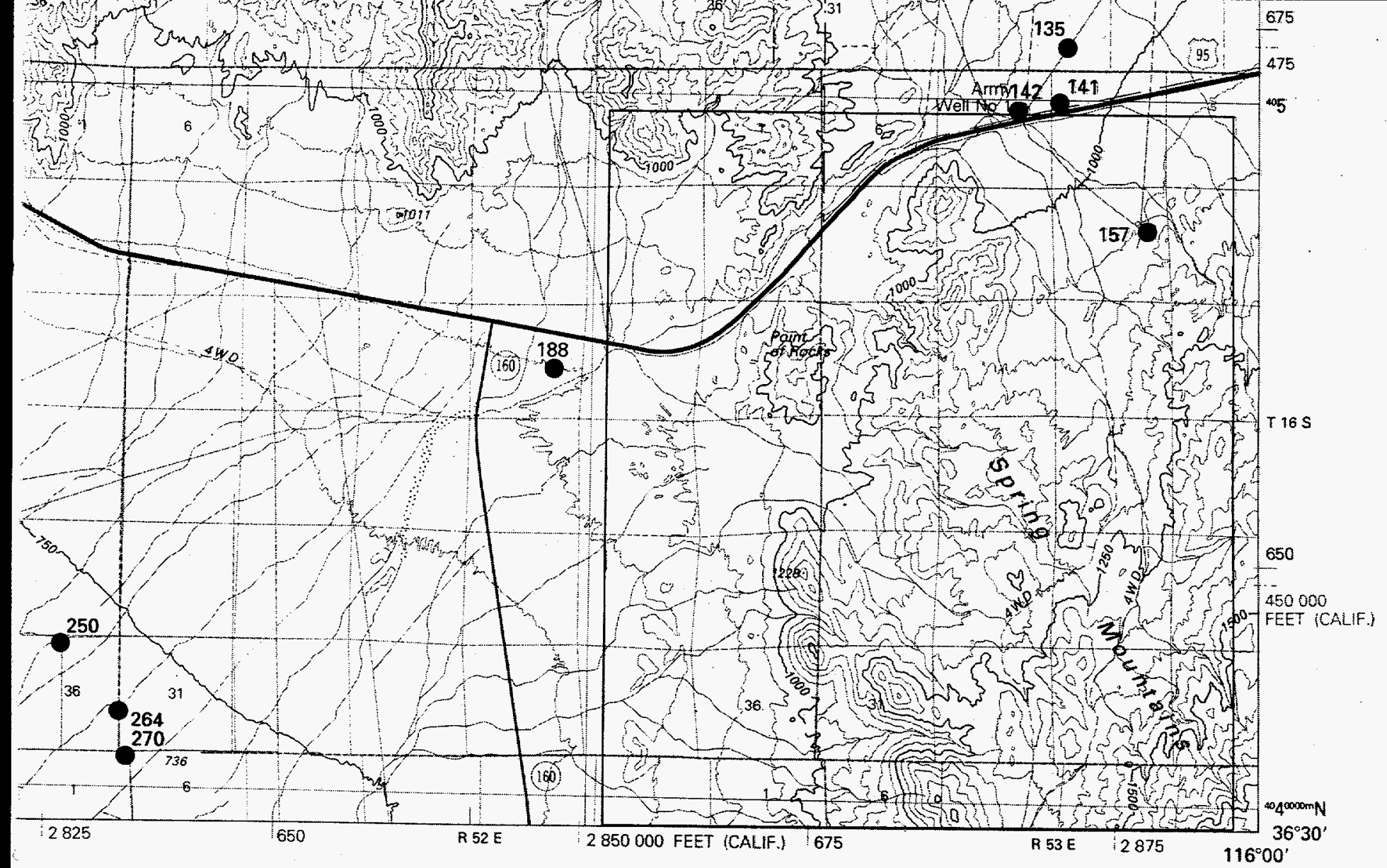

I000 FEET 


\section{U.S. DEPARTMENT OF THE INTERIOR U.S. GEOLOGICAL SURVEY}

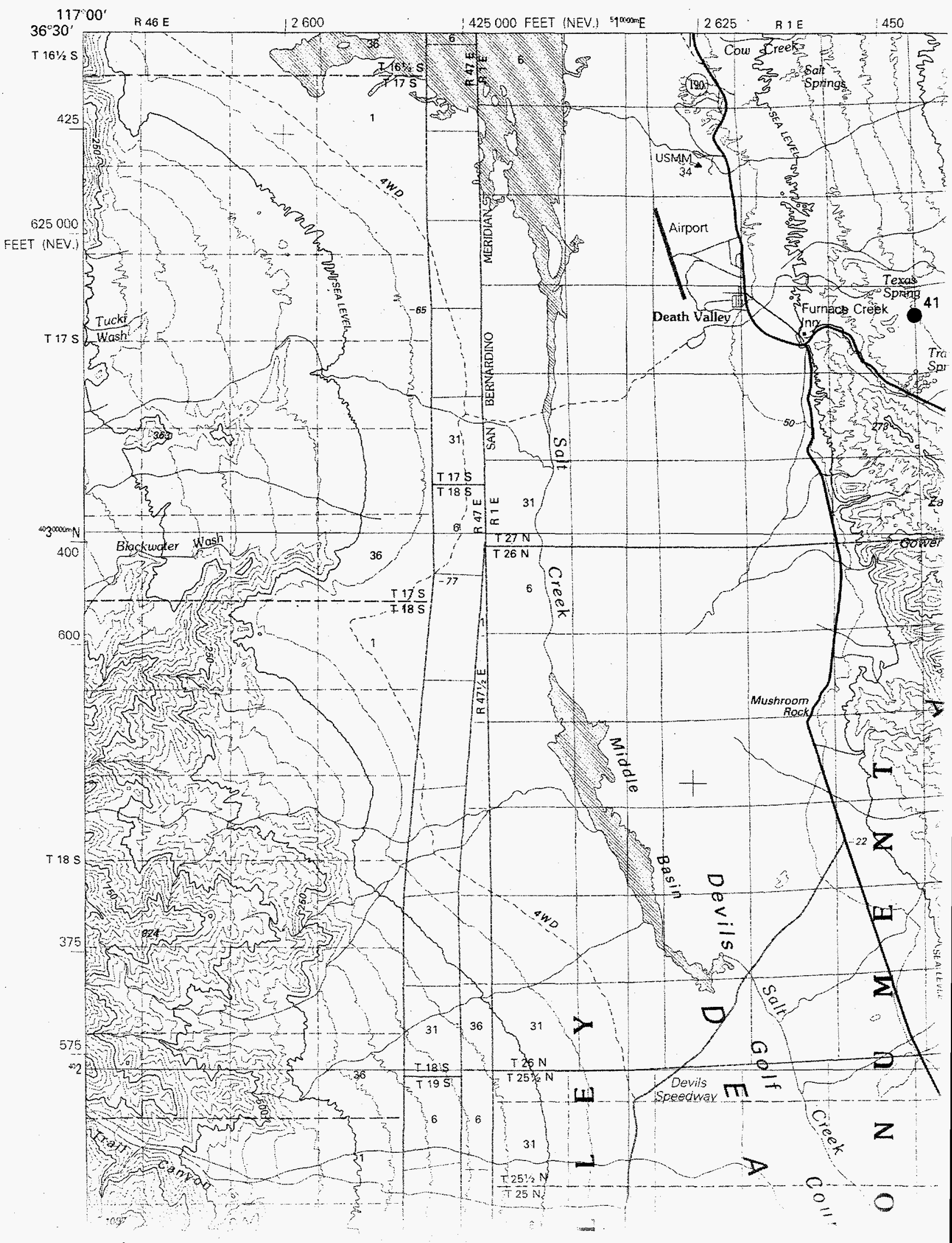




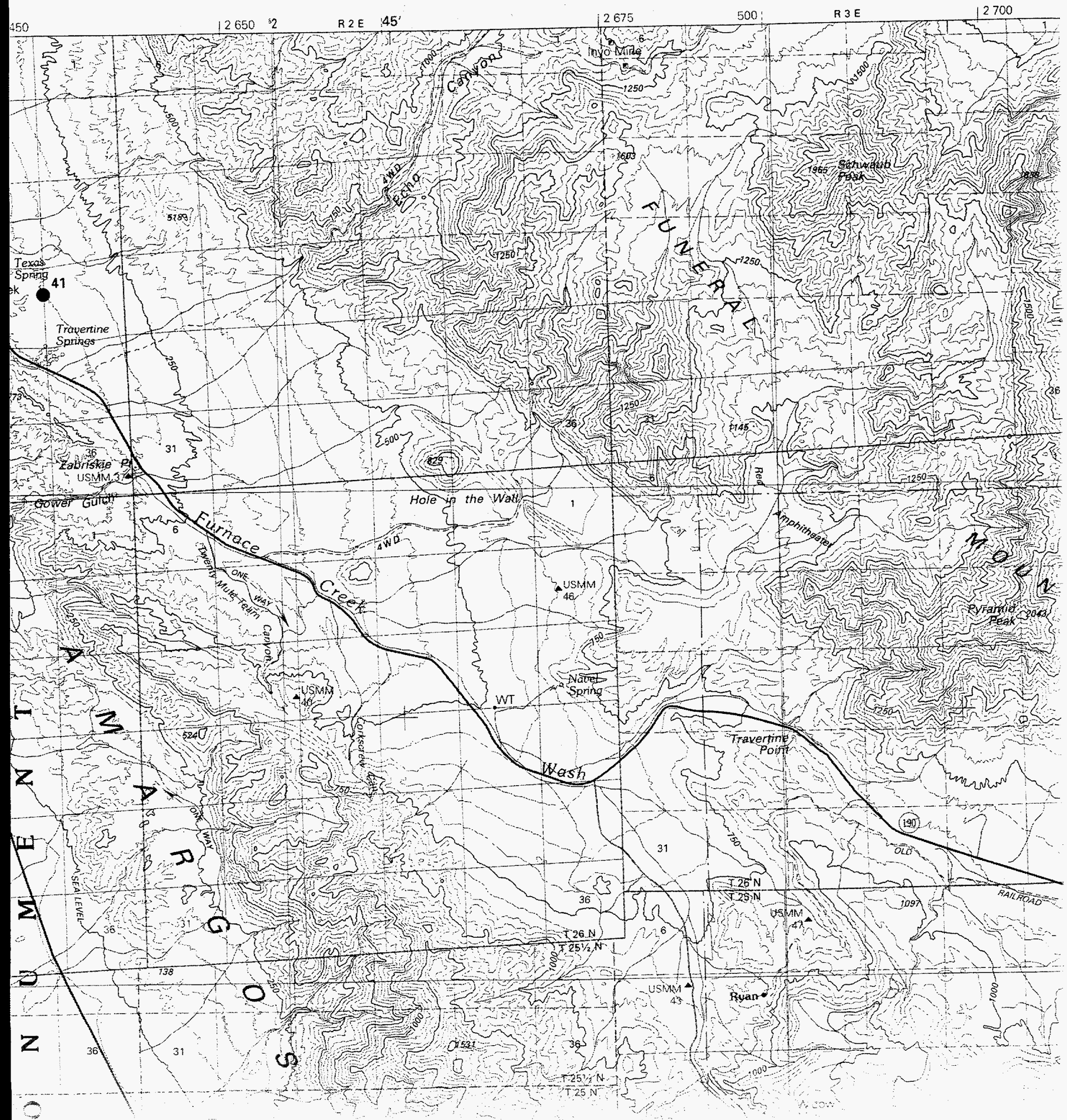




\section{Prepared in cooperation with the U.S. DEPARTMENT OF ENERGY NEVADA OPERATIONS OFFICE}

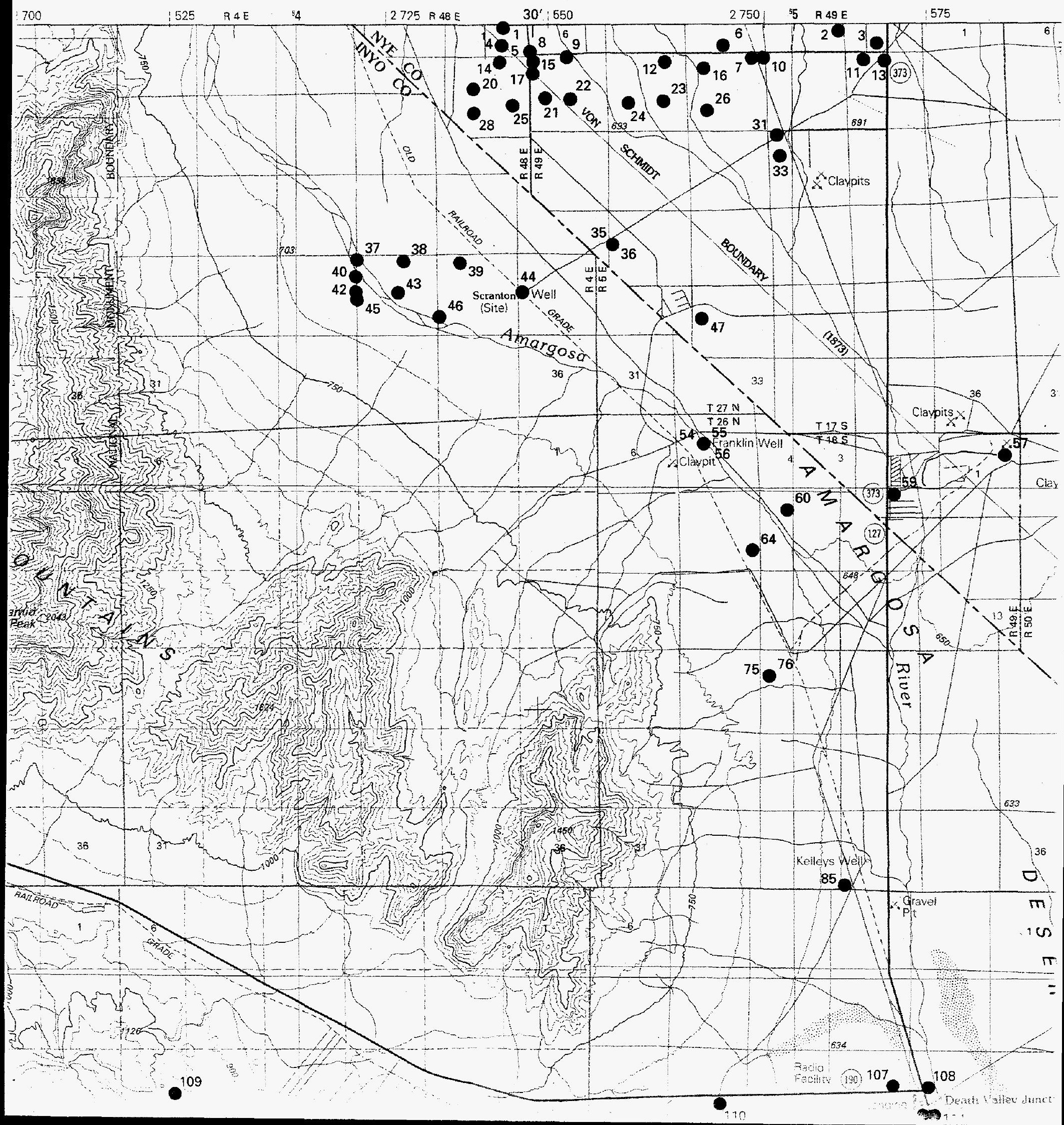




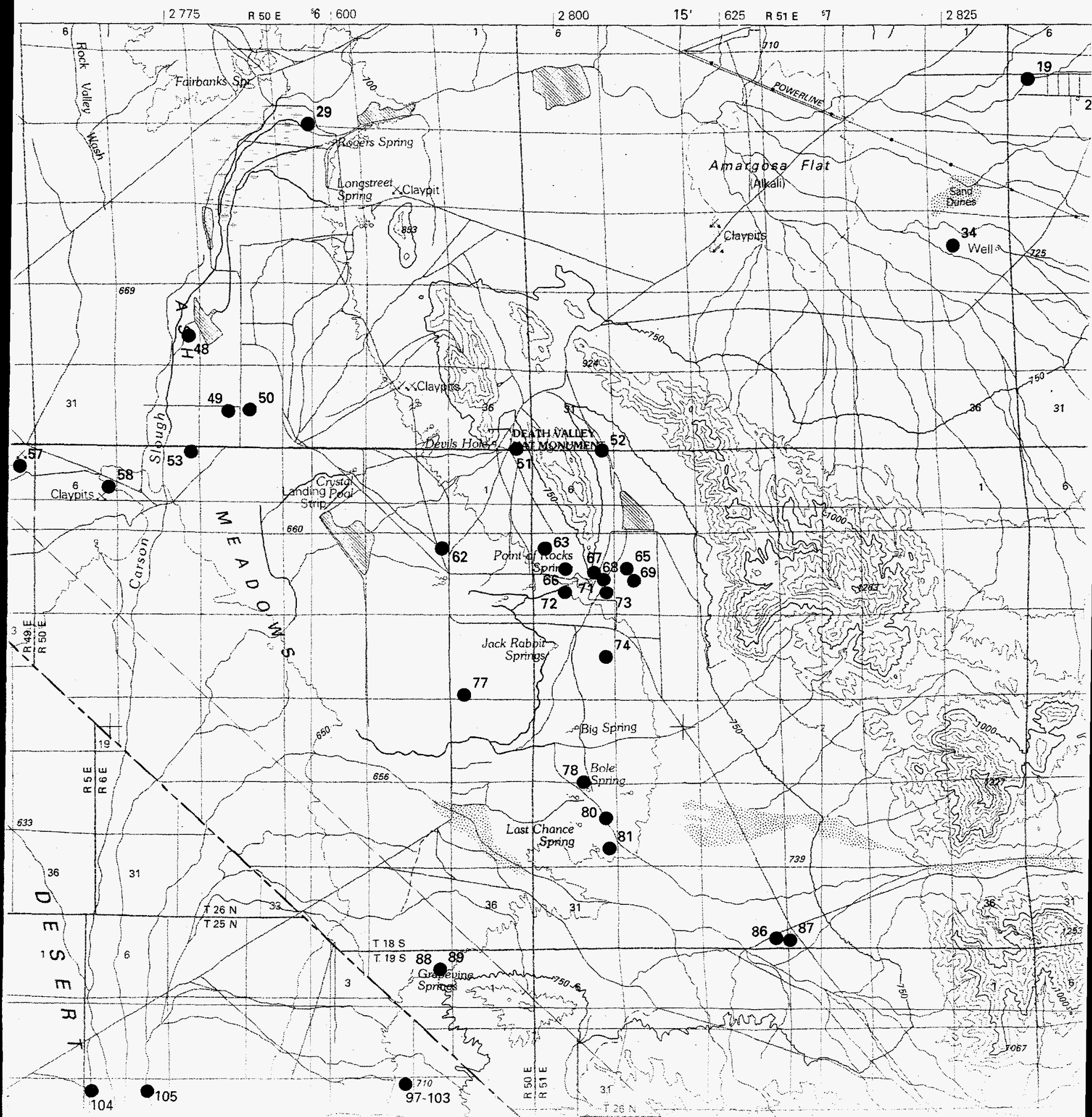




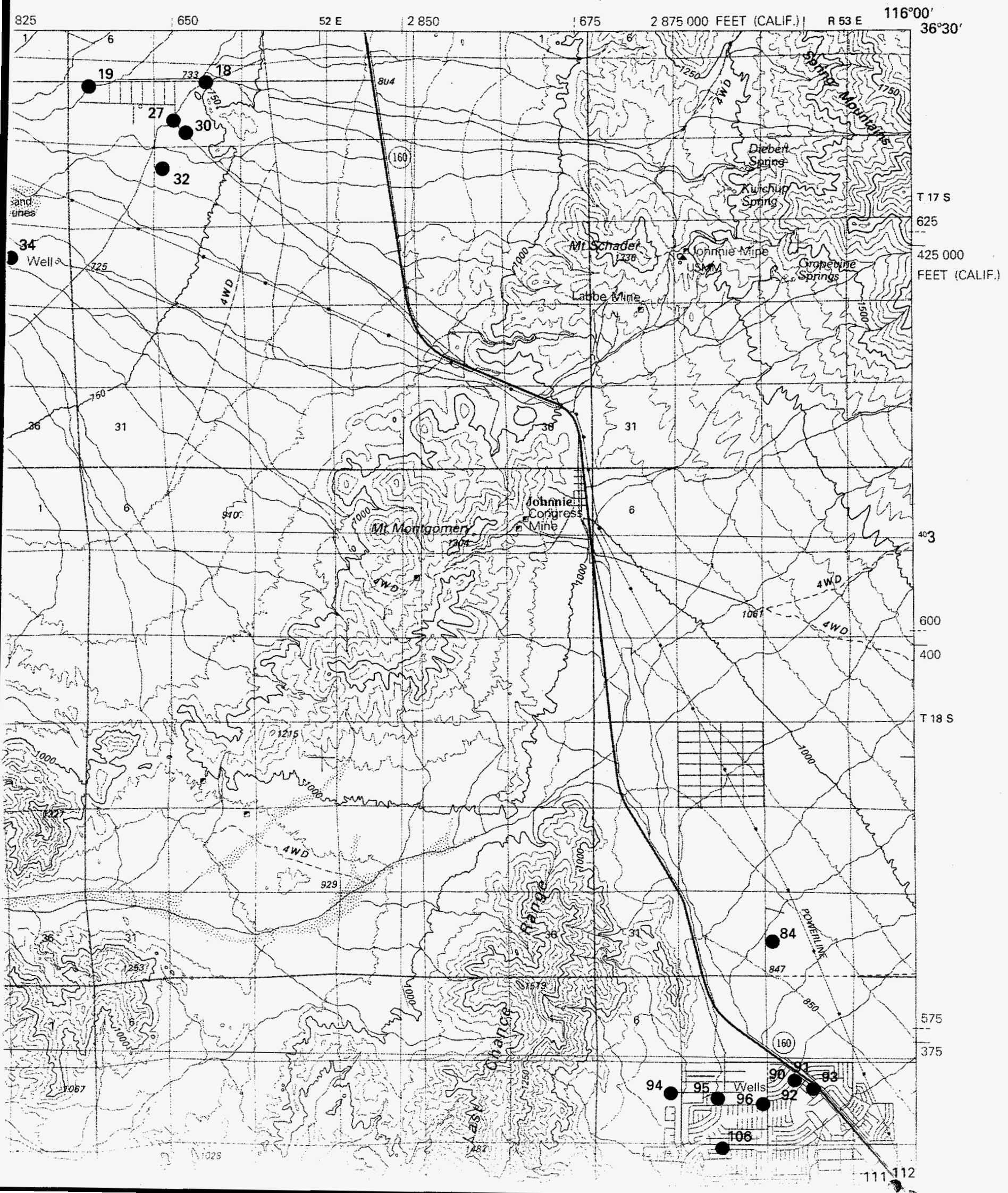




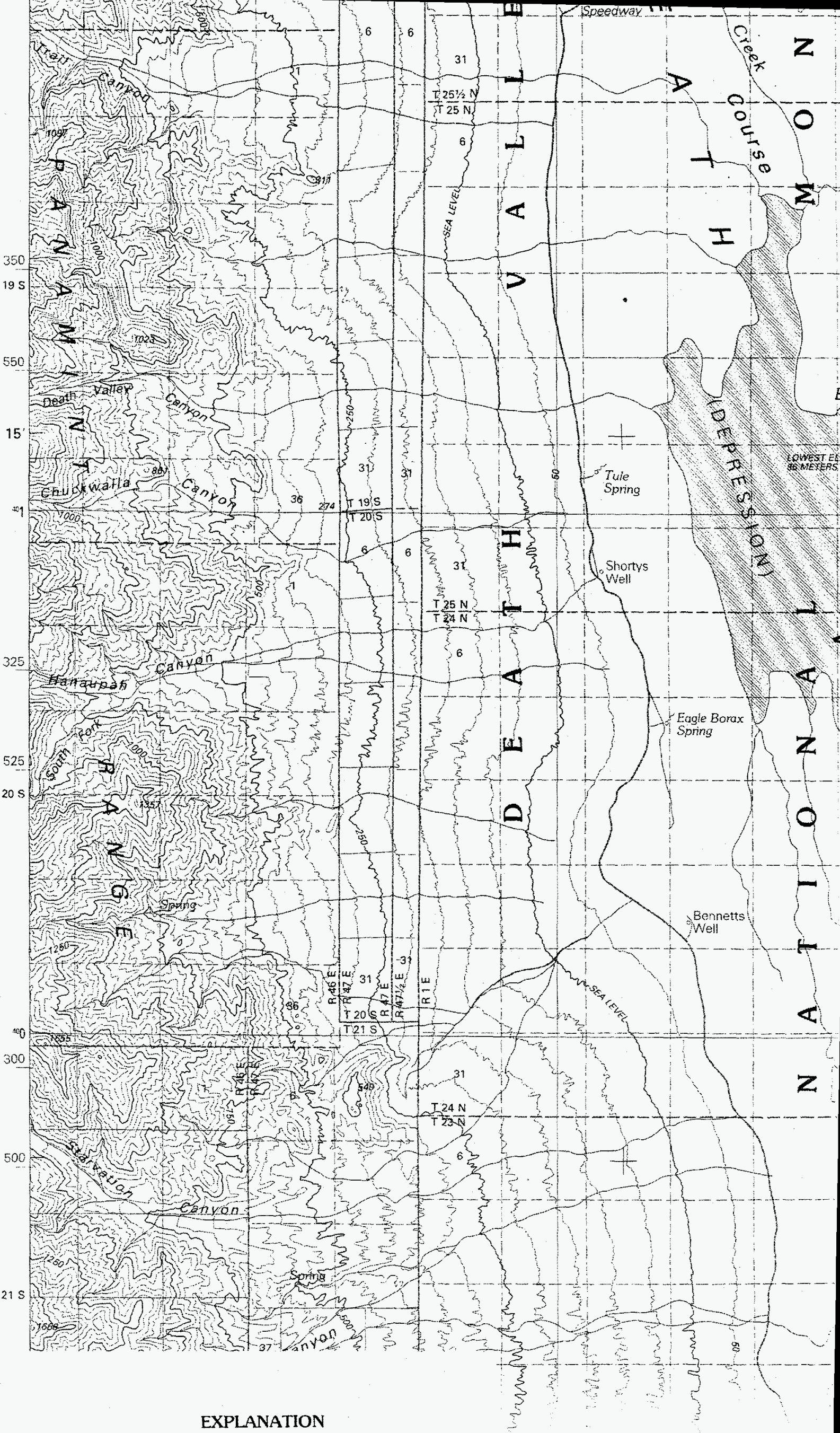




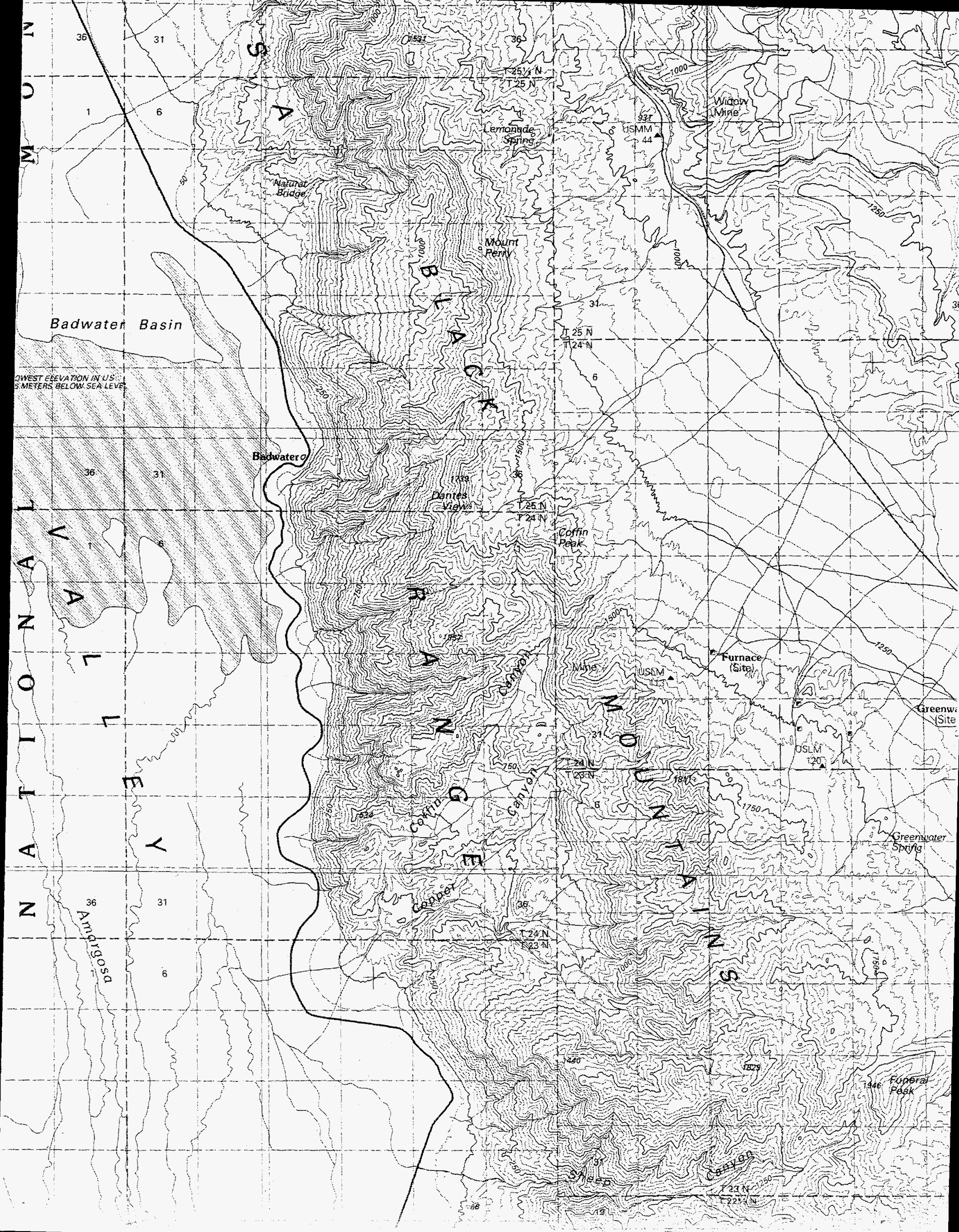





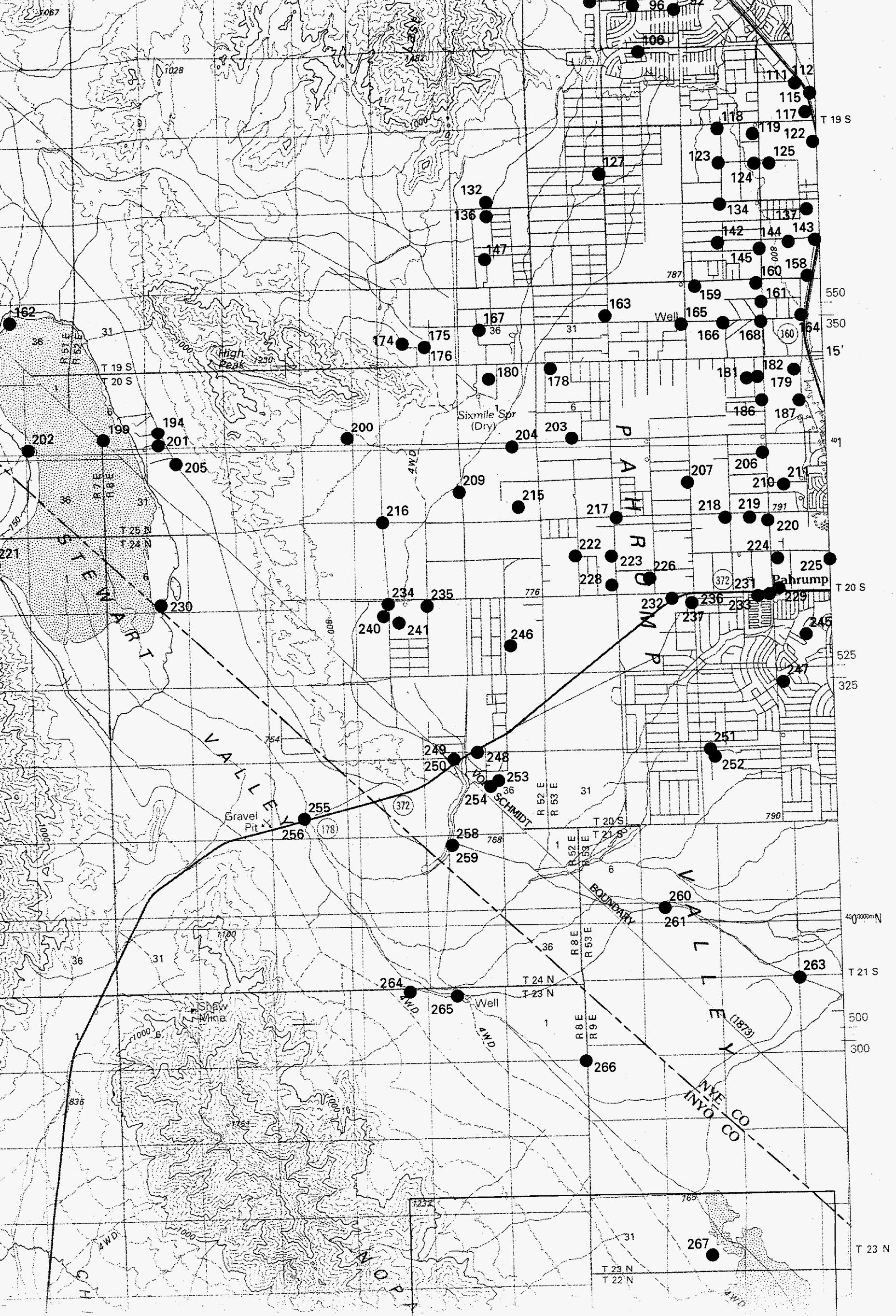




\section{EXPLANATION}

$0^{160}$ WELL LOCATION-Numbers correspond to the site numbers in tables 1 and 2 in the report.

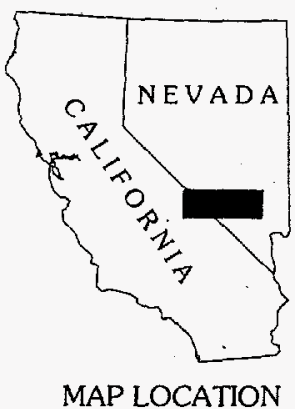

T $22 \mathrm{~S}$

$36^{\circ} 00^{\prime}$

$117^{\circ} 00^{\prime}$
R $47 \mathrm{E}$
R $46 E$

Base from U.S. Geological Survey

$1: 100,000$, Death Valley Junction

California-Nevada, 1986

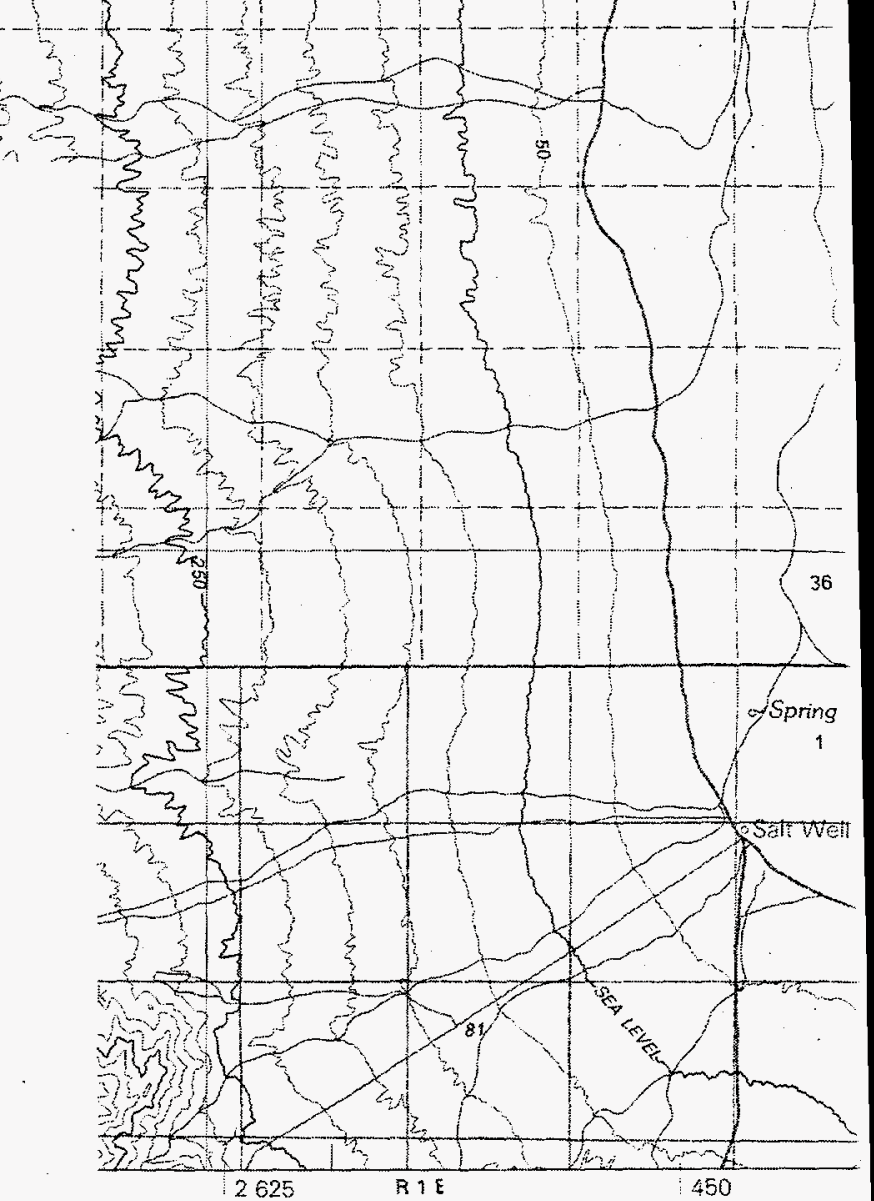




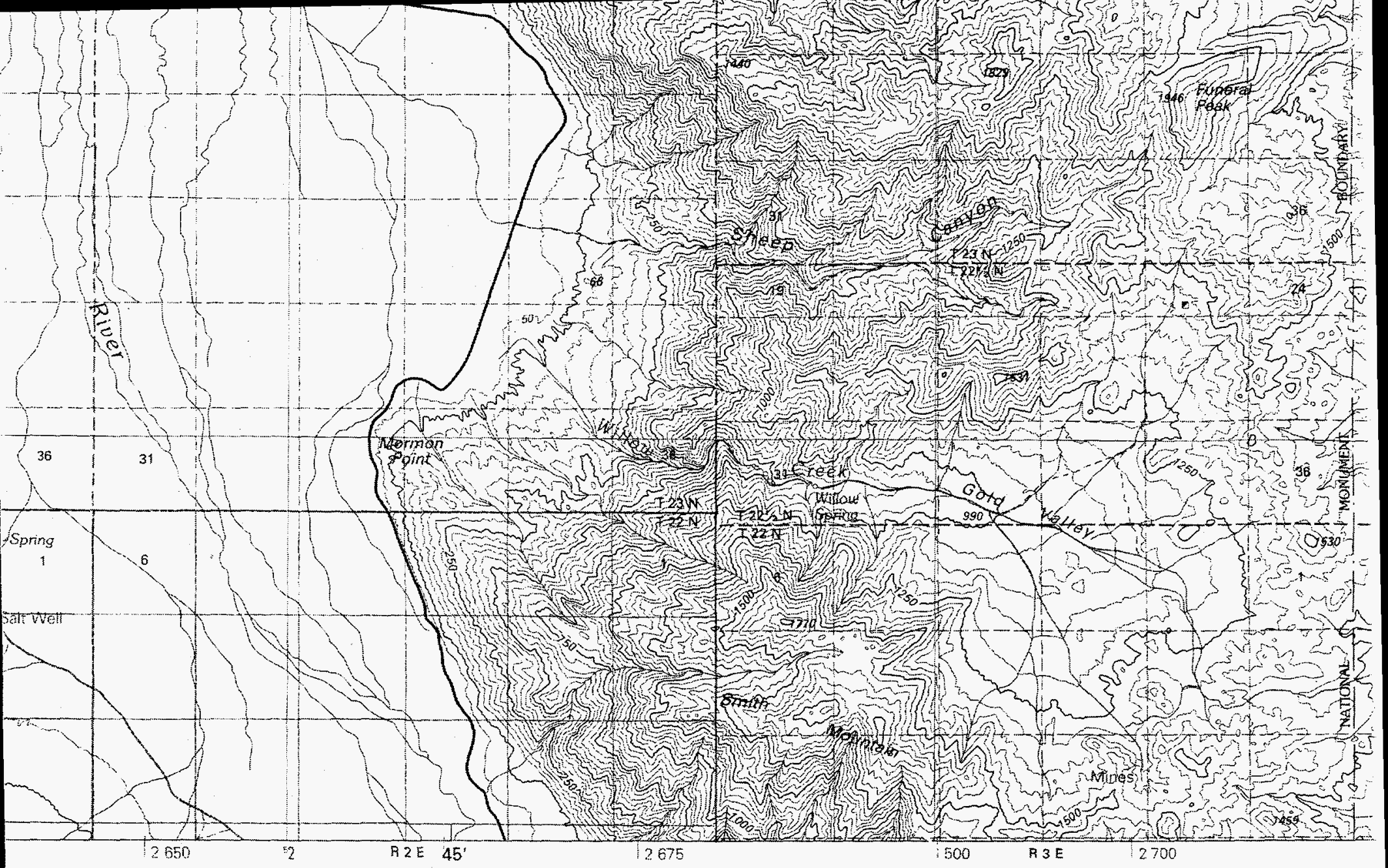

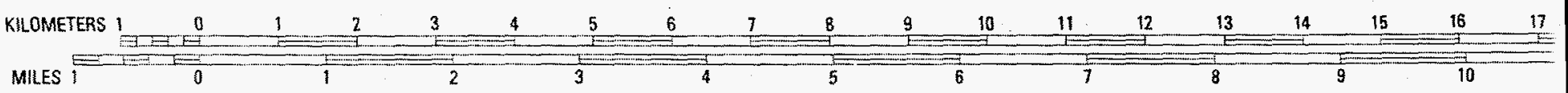




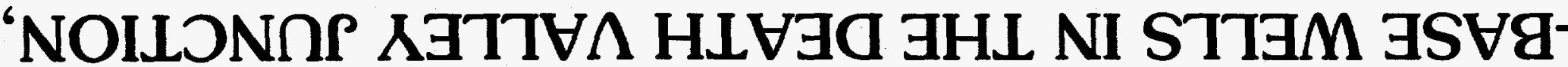

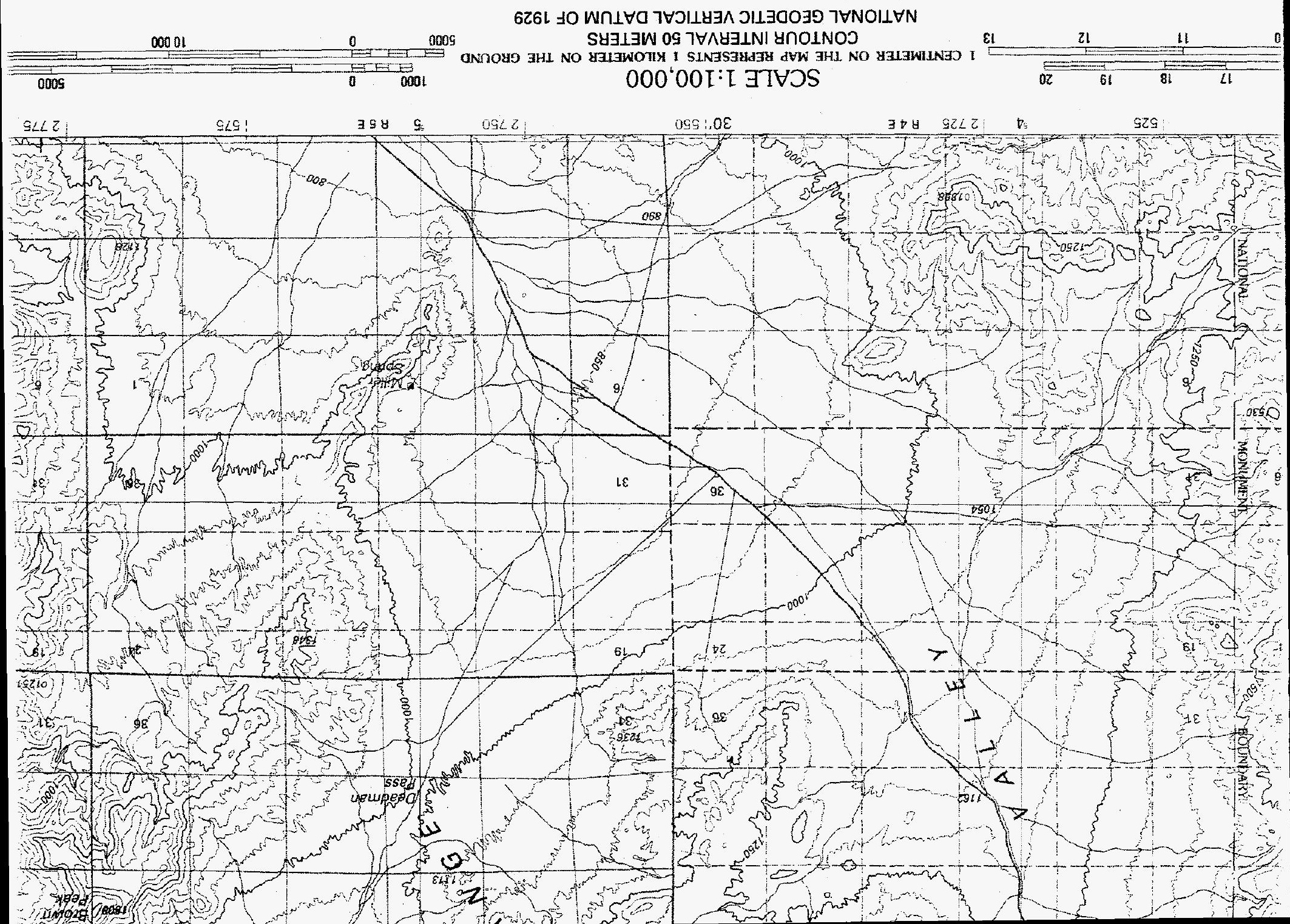




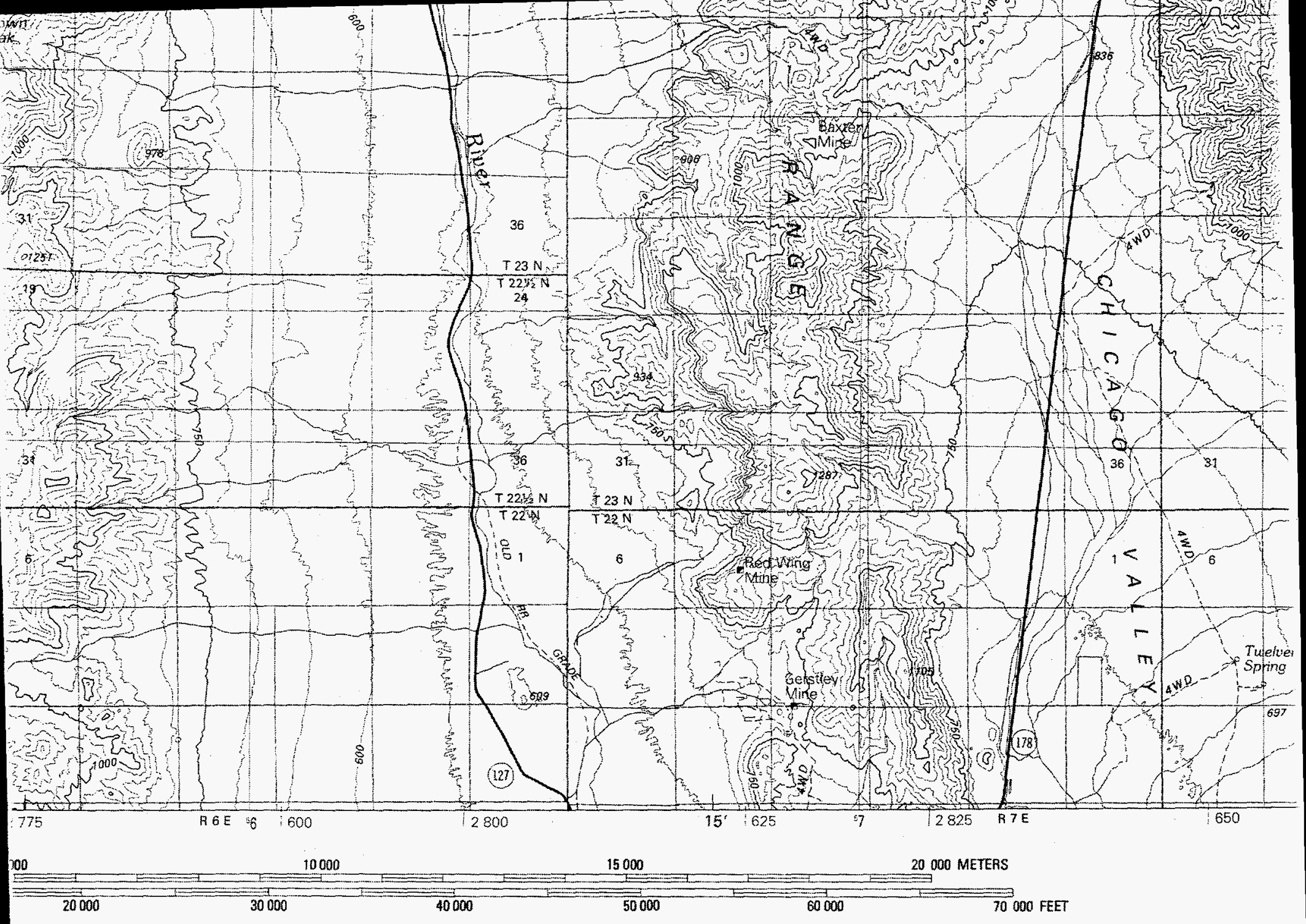

\section{I, CALIFORNIA-NEVADA QUADRANGLE}




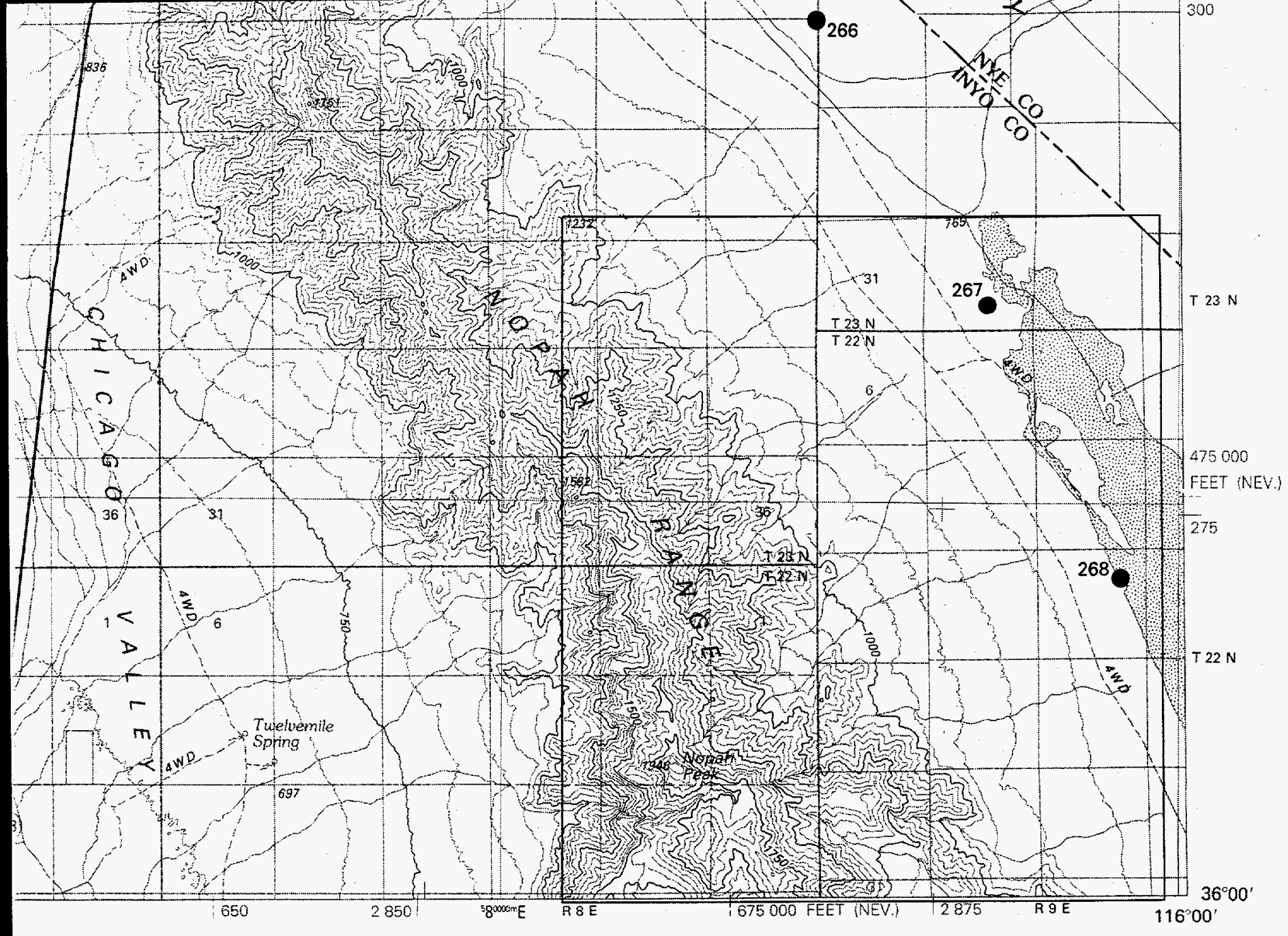

